CONFERENCE PROCEEDINGS : Abstracts for the "Evolutionary Medicine Conference: Interdisciplinary Perspectives on Human Health and Disease" at the University of Zurich, Switzerland (July 30-August 1, 2015)

Edited by: Staub, Kaspar ; Bender, Nicole ; Ewald, Paul ; Rühli, Frank J

DOI: https://doi.org/10.4303/jem/235924

Posted at the Zurich Open Repository and Archive, University of Zurich ZORA URL: https://doi.org/10.5167/uzh-123337

Edited Scientific Work

Published Version

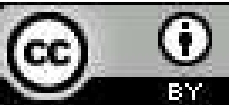

The following work is licensed under a Creative Commons: Attribution 3.0 Unported (CC BY 3.0) License.

Originally published at:

CONFERENCE PROCEEDINGS : Abstracts for the "Evolutionary Medicine Conference: Interdisciplinary Perspectives on Human Health and Disease" at the University of Zurich, Switzerland (July 30-August 1, 2015). Edited by: Staub, Kaspar; Bender, Nicole; Ewald, Paul; Rühli, Frank J (2015). Roisin, Honnelles, Belgium: Ashdin.

DOI: https://doi.org/10.4303/jem/235924 


\title{
Abstracts for the "Evolutionary Medicine Conference: Interdisciplinary Perspectives on Human Health and Disease" at the University of Zurich, Switzerland (July 30-August 1, 2015)
}

\author{
Kaspar Staub, ${ }^{1}$ Nicole Bender, ${ }^{2}$ Paul Ewald, ${ }^{3}$ and Frank Rühli ${ }^{1}$ \\ ${ }^{1}$ Institute of Evolutionary Medicine, University of Zurich, Winterthurerstrasse 190, CH-8057 Zurich, Switzerland \\ ${ }^{2}$ Institute of Social and Preventive Medicine, University of Bern, Finkenhubelweg 11, CH-3012 Bern, Switzerland \\ ${ }^{3}$ Department of Biology, University of Louisville, Louisville, KY 40292, USA \\ Address correspondence to Frank Rühli,frank.ruehli@iem.uzh.ch
}

Received 17 Aril 2015; Revised 11 May 2015; Accepted 14 May 2015

Copyright (C) 2015 Kaspar Staub et al. This is an open access article distributed under the terms of the Creative Commons Attribution License, which permits unrestricted use, distribution, and reproduction in any medium, provided the original work is properly cited.

Summary In summer 2015, the "Evolutionary Medicine Conference 2015: Interdisciplinary Perspectives on Human Health and Disease" takes place at the Institute of Evolutionary Medicine, University of Zurich, Switzerland. This international conference is the first of its kind in Europe and brings together eight distinguished keynote speakers from all over the world as well as experts from different disciplines (including medicine, anthropology, molecular/evolutionary biology, paleopathology, archeology, history, psychology, epidemiology, and other fields) to debate the evolutionary origins of diseases and on how the knowledge of the past informs the present and the future. In addition, the conference programme includes round tables on how to bring Evolutionary Medicine into the medical curriculum and clinical practice, and on the future and the challenges of the field. This single article contains all accepted abstracts for keynote lectures, minisymposia, oral and poster presentations (peer-reviewed and accepted by the scientific committee as of May 2015). This collection provides a sense of the breadth of evolutionary medicine in this year of its development. But it also brings into focus the challenge of applying and integrating the wide variety of research areas and perspectives using the conceptual framework provided by evolutionary thinking.

Key to abbreviations __ : The submitting/presenting author is underlined; K: Keynote presentation; MS: Minisymposium; O: Oral presentation; P: Poster presentation

K-01: The central challenge for evolutionary medicine: deciphering the interplay between roles of genes, germs, and the environment

$\underline{\text { P. Ewald }}$

Department of Biology, University of Louisville, USA E-mail:pw.ewald@louisville.edu

Keywords: Chlamydia pneumoniae; atherosclerosis; epsilon 4

Evolutionary medicine attempts to understand how and why individuals develop illness and to apply this understanding to improve states of health. Studies in evolutionary medicine generally have evaluated particular hypotheses for particular diseases. The discipline is now at a turning point at which a rigorous application of evolutionary insights to the medical sciences will require not only assessing the validity of the full spectrum of possible explanations for each disease but the interplay of different contributors to illness within and between the three broad categories of causal factors: genetic, infectious, and environmental. This presentation uses atherosclerosis to illustrate the need for this integrative approach, by evaluating the interplay between three risk factors drawn from these three categories: the epsilon 4 allele, Chlamydia (= Chlamydophila) pneumoniae, and cigarette smoking. The analysis emphasizes the inadequacy of considering each risk factor in isolation and the how an integrated understanding may lead to better management of this disease.

K-02: Medicine without evolution is like engineering without physics

\section{$\underline{\text { R. Nesse }}$}

The Center for Evolution \& Medicine, Arizona State University, USA

E-mail:nesse@asu.edu

Keywords: evolutionary medicine; biology; why we get sick

Evolutionary medicine applies the principles of evolutionary biology to the problems of medicine. Like other basic sciences, it guides research and provides a framework for understanding disease. However, it is even more essential for understanding why bodies usually work so well and why they are nonetheless vulnerable to failure. This talk will outline six reasons why bodies are vulnerable to disease. The emphasis will be on the principle that selection does not 
shape bodies for health, and on the principle that regulation mechanisms that adjust in response to experience are inherently vulnerable to failure from runaway positive feedback.

\section{K-03: Milk and serum factors as risk for colon and breast cancer}

\section{$\underline{\text { H. zur Hausen }}$}

\section{German Cancer Research Center, Heidelberg, Germany E-mail:zurhausen@dkfz-heidelberg.de}

Keywords: cancer; infection

Presently, approximately $21 \%$ of the global cancer incidence has been linked to infectious events, involving viral, bacterial, and parasitic infections. A number of viral, bacterial, and parasitic diseases originate from transmissions of infections by contacts with domestic animals or their products. Several chronic, latent, and persistent infections prevalent in human populations are not or only rarely causing symptoms in the human host. Some of them, however, are relatively effective inducers of malignant tumors or of chronic neurological disease in rodents or New World primates, commonly under conditions not allowing their replication. This prompted the consideration whether similar agents exist in domestic animals (cattle), not tumorigenic in their native hosts, but potentially causing cancer in a nonpermissive host, after infection of humans. Based on global epidemiological data, prime candidates for these studies are human colon and breast cancers, the former clearly linked to beef consumption, the latter seems to be linked to cow milk uptake at early age. This resulted in the postulate of potentially oncogenic infectious factors for colon cancer in beef, probably interacting synergistically with some of the chemical carcinogens, produced in preparatory steps for consumption. Similar but not identical factors have been postulated in milk contributing to the risk of breast cancer. This resulted in the analysis of sera and milk from healthy cattle for the presence of infectious agents interacting with human cells. Twenty novel agents (circular single-stranded, episomally persisting DNAs) have been isolated and characterized by now, most of them from cattle sera, several directly from pasteurized milk. A few other types originated directly from human materials. The available results will be presented.

\section{K-04: Consequences of changing demographic structure and dynamics for future evolution of human health}

\section{Henneberg}

\section{Medical School, University of Adelaide, Australia \\ E-mail: maciej.henneberg@adelaide.edu.au}

Keywords: demography; mortality; fertility; evolution

During the late 20th century, profound changes in the demographic dynamics of the large number of national populations took place. This has consequences for population structure by sex and age. A large part of those changes resulted from public health and medical advances, but, inversely, they will have an influence on the health of future generations. Changes in mortality and fertility reflect altered opportunities for natural selection while varying migration intensity and patterns provide for differences in gene flow and spread of pathogens. Worldwide newborn life expectancy increased by over 20 years (about 50\%) from 1950s to 2010-2015. In 39 countries, a newborn has at least $99 \%$ opportunity to pass its genes to the next generation without a threat of premature mortality. Total fertility has decreased below the simple replacement level in over one third of nations, especially those most developed ones. The decline in fertility is a result of voluntary birth control unrelated to biological determinants of fertility. Decline in mortality, and in fertility, greatly reduces opportunity for the action of natural selection, while with the world population exceeding 7 billion, and with scores of de novo mutations appearing in each newly conceived individual, the mutationselection balance is strongly biased towards the accumulation of mutations. Many of those mutations are neutral, but the probable effect of random mutations is a deterioration of the functions and structures of organisms. Increasing life expectancy results in larger proportions of postreproductive age people in a population. Postreproductive life is characterized by functional inadequacies as a result of past adaptations based on differential reproductive success, that, obviously cannot adjust those characteristics of the human body that are important after reproductive activities have ceased. The new demographic situation already observed in a large portion of nations will spread throughout the $21 \mathrm{st}$ century as the economic and societal life of other nations improves. Since maintenance of human life is a paramount principle, health services will have to carry an increasing burden of corrective procedures. It is important to plan for this now so that national economies will be able to provide adequately for the progressively greater costs of maintaining human health, or new approaches, replacing forces of evolution, will be developed.

\section{K-05: Developmental perspectives in evolutionary medi- cine}

G. Bentley

Department of Anthropology, Durham University, $U K$

E-mail: g.r.bentley@durham.ac.uk

Keywords: childhood; developmental factors; reproductive phenotypes

Perspectives on early life development are prominent within evolutionary medicine, but mainly focus on events in utero or infancy. My work has concentrated on establishing the importance of the entire childhood period for influencing reproductive phenotypes. I argue that, given our species' 
long lifespan and the critical importance of reproductive function for individual reproductive success, humans should track environmental parameters for as long as possible before committing the phenotype to particular adult levels of reproductive robustness. Together with my research team, we have run a series of studies using migrant Bangladeshis in the UK as a model for examining the role of the childhood environment in influencing various aspects of reproductive function, including hormone levels, length of the reproductive lifespan, and rates of ovulation. We have found that Bangladeshi women who spent their childhood in Bangladesh (where they are challenged by high rates of infectious disease and a poor health service) have a later age at menarche, an earlier age at menopause, lower levels of reproductive steroids, lower rates of ovulation, and a lower age-matched ovarian reserve compared to either Bangladeshi women who spent their childhood in the UK, or UK women of European origin. Bangladeshi men who spent their childhood in Bangladesh have lower levels of salivary testosterone compared to migrants who grew up in London, or UK men of European origin. Women who migrated to the UK before age eight had more robust indicators of reproductive function compared to migrants who arrived between ages nine and sixteen. In a further study of Bangladeshi children, first-generation early child migrants reached adrenarche two years earlier than second-generation migrants or European children. Apart from being important for reproductive biology, these data suggest that other physiological systems may be similarly influenced by the environment experienced during the entire childhood period.

\section{K-06: Birth, obstetrics, and evolutionary medicine}

\section{$\underline{\text { W. Trevathan }}$}

Department of Anthropology, New Mexico State University, USA

E-mail:wtrevath@nmsu.edu

Keywords: childbirth; evolutionary medicine

Few points in the life cycle are subject to more intense evolutionary forces than the moment of birth, when both survival and reproductive success are at stake. The challenges are particularly great for species in which the birth canal and neonatal head size show close correspondence, a phenomenon that describes most primates. This includes our own species, but for humans, anatomical changes for bipedalism restructured the pelvis in ways that altered the birth mechanism, requiring a series of fetal rotations. Additional challenges to the birth process occurred with dramatically increased brain size beginning about two million years ago. Although complications at birth likely increased significantly with agriculture and, in recent years, with increased reliance on medical technology, there are a number of phenomena associated with childbirth that may benefit from consideration by evolutionary medicine. For example, with a bipedal pelvis, the infant tends to emerge facing away from the mother, placing a selective premium on having a companion present to help guide the infant out and wipe fluids from the face. When a woman seeks companionship due to fear and anxiety she is engaging in a positive response to what some may see as a defect (fear that interferes with contractions) rather than a defense (soliciting assistance at birth). Other aspects of birth that can be considered from the viewpoint of evolutionary medicine include shoulder dystocia, cephalopelvic disproportion, pelvic organ prolapse, and skyrocketing rates of cesarean section. Examining birth through the lens of evolutionary medicine invites consideration of evolutionary mismatches, life cycle trade-offs, watchful waiting, defenses and defects, lack of perfection in design, and parent-infant conflict. Perhaps most importantly, evolutionary medicine may lead to alternatives to cesarean section, a medical intervention that has significant risks to both mother and infant, including epigenetic changes that may have transgenerational effects.

\section{K-07: In the neighborhood: Local norms and ecological} effects in health-related and reproductive behaviors

R. Mace, C. Uggla, and B. van Lueewen

UCL Anthropology, University College London, UK

E-mail:r.mace@ucl.ac.uk

Keywords: reproductive behavior; local norms and ecology

Health-related behaviors are influenced by the environment in which people live. Life-history theory predicts that risky environments may increase emphasis on early reproduction and reduce emphasis actions with long-term benefits. Cultural evolutionary models also predict that some of our behavior is influenced by what others around us choose to do, which could also potentially generate different local norms of behavior. Using datasets from both the UK (Northern Ireland and England), Europe, and Africa, we evaluate the evidence for and correlates of local norms of behaviors including age at first sex, first birth, violence, and aspects of parental care. We conclude that local ecology has large effects on human behavior, a small proportion of which is explained at the area level once individual effects such as wealth have been controlled for.

\section{K-08: Major themes in evolutionary medicine}

\section{S. Stearns}

Department of Ecology \& Evolutionary Biology, Yale University, $U S A$

E-mail: stephen.stearns@yale.edu

Keywords: evolutionary medicine; pathogen-host interaction; antibiotic resistance; cancer treatment

I will start by describing the scope of evolutionary medicine as I understand it. In this introduction, I will also mention 
several key issues: differing styles of thought, what we think a patient is, whether organisms should resist a pathogen or tolerate it, whether imperfect vaccines will have the unfortunate side effect of causing an evolutionary increase in pathogen virulence, whether insights gained from the phylogenetic analysis of cancer open doors to new approaches to treatment, and whether individual medical decisions have important population consequences.

I will then explore two issues with clinical applications in greater detail: how to manage and even avoid the evolution of antibiotic resistance, and how to manage the evolution of resistance to chemotherapy by cancers. I also discuss another potential application suggested by the Hygiene Hypothesis: the use of worm therapy to cure autoimmune disease. Here the results are not yet clear, but there is promise. I will end with an interesting insight into the unexpected connection between invasive placentation and the risk of metastatic cancer.

My overall conclusion will be that evolutionary thinking casts new light on many areas of medical practice and research. In doing so, it complements other approachesit does not replace them. It has a considerable potential to reduce suffering and save lives, and it contains fascinating ideas.

MS-01: Public health reform, sanitary investment, and the decline of mortality in the late 19th and early 20th centuries

Organizer: B. Harris

School of Social Work and Social Policy, Faculty of Humanities and Social Sciences, University of Strathclyde, Glasgow, Scotland UK

E-mail: bernard.harris@strath.ac.uk

Towards the end of the 19th century, mortality rates began to decline in many European countries. In a series of publications, Professor Thomas McKeown, Professor of Social Medicine at the University of Birmingham, argued that this decline was driven primarily by improvements in diet and the standard of living, and only secondarily by improvements in the public health infrastructure. This claim has often proved controversial. For example, in 1988, the British historian, Simon Szreter, argued that McKeown had exaggerated the importance of the role played by nutritional improvements and paid insufficient attention to the role played by sanitary reform. Szreter's paper has had a powerful influence on subsequent contributions to this debate in both medical history and epidemiology. Much of this argument continues to depend on inferences from some fairly broad-brush data. However, a number of economists, historians, and demographers are now seeking to reopen the debate by conducting much more detailed analyses of the relationship between sanitary expenditures and mortality changes in a range of different countries. This session brings together three such papers. In the first paper, Bernard Harris and Andrew Hinde examine the chronology and geography of the loans contracted by local authorities for sanitary and other purposes in England and Wales between circa 1850 and 1914. In the second paper, Jose Joaquin Garcia Gomez, Javier Puche Gil, and Eva Maria Trescastro Lopez explore the impact of public health expenditure on biological welfare during the period of Spanish industrialization. In the third paper, Kaspar Staub, Joel Floris, Frank Rühli, and Ulrich Woitek look at the impact of sanitary reforms on mortality in different parts of Switzerland. Taken together, the three papers aim to shed some much-needed new light on the details of sanitary improvement in this period and their impact on mortality change.

Speakers:

O-02: J. Garcia Gomez, J. Puche Gil, and E. Trescastro Lopez: Expenditures in public health and biological welfare during the Spanish industrialization (1840-1914).

O-31: K. Staub, J. Floris, F. Rühli, and U. Woitek: Selected sanitary reforms in Switzerland and their impact on mortality during the 19 th and 20th centuries-A cantonal analysis.

O-49: B. Harris and A. Hinde: Public works loans, social intervention and mortality change in England and Wales, 1850-1914.

\section{MS-02: An evolutionary perspective on human adiposity}

Organizer: J. Wells

Institute of Child Health, University College London (UCL), London, UK

E-mail: jonathan.wells@ucl.ac.uk

The obesity epidemic has focused clinical attention on the health penalties for excess fatness. This is one of the most significant public health challenges of modern times, given the association of obesity with multiple chronic diseases including diabetes, stroke, hypertension, heart disease, dementia, and many cancers. From an evolutionary perspective, however, obesity represents the perturbation of a trait that is fundamentally adaptive-body fat. To improve understanding of human susceptibility to obesity, we need to first understand how body fat evolved to respond adaptively to ecological stresses, and then identify how to minimize the perturbation of such plasticity. Although epidemiological work and clinical trials have already identified many risk factors for obesity, an integrated perspective is still emerging.

This minisymposium will offer three related presentations on the adaptive benefits of body fat in humans. The first presentation will focus on adipose tissue as a "risk management" organ, working in concert with the brain. Body 
fat will be shown to manage life history functions so as to optimize the balance between survival and fitness. The second presentation will report novel work using mathematical modeling, elucidating how the ratio of adipose tissue to lean tissue varies in response to ecological factors under the pressure of maximizing fitness. The third presentation will focus on a specific human dataset, disentangling secular trends in weight and height in Swiss conscripts over 130 years, in order to elucidate how plasticity in size evolves across generations.

\section{Speakers:}

O-19: R. Johnstone, A. Rodrigues, and J. Wells: Optimal fat storage and the risk of diabetes.

O-32: J. Wells: Untangling our predisposition to obesity: adipose tissue as an organ of risk management.

O-55: K. Staub, N. Bender, and F. Rühli: Secular trends in weight and height and their interrelationship in Swiss conscripts over 130 years.

\section{MS-03: A cross-species, evolution-based system for char-} acterizing disease

Organizers: E. Uhl ${ }^{1}$ and N. Warner ${ }^{2}$

${ }^{1}$ Department of Pathology, College of Veterinary Medicine, University of Georgia, USA

${ }^{2}$ Warner Consulting, USA

E-mail:euhl@uga.edu

To date, diseases have been classified and studied largely based upon observable findings or phenotypes including clinical signs and symptoms, pathology, and response to treatment. Advances in technology now allow genotypic and bioinformatic information to be incorporated into disease definitions. This shifting paradigm is leading to a more evolutionary-based approach to medicine that will enhance our understanding of what constitutes "health" and "disease." Given that few disease phenotypes are truly unique to humans, and that genome sequencing has revealed substantially greater genetic homology between man and animals than was expected, a logical focus for biology and medicine is to devise an integrated disease classification system that incorporates both animal and human diseases. A classification system that uses genetic information applied in an evolutionary context will improve understanding of and communication about diseases, particularly complex diseases, in ways that will improve diagnoses, yield new targets, and translate to novel therapeutic and preventive interventions for human and veterinary medicine. This minisymposium will discuss the limitations of the current disease definitions and advantages of a cross-species, evolution-based system for classifying disease from medical, veterinary, and biomedical research perspectives. Discussion will focus on ways to implement an evolution-based definition of disease.

Speakers:

O-40: E. Uhl: Redefining disease: importance of an evolutionary cross-species perspective.

O-41: N. Warner: Failing hearts and failed definitions.

O-01: Postnatal depression and reproductive success in modern, low-fertility contexts

S. Myers, O. Burger, and S. Johns

School of Anthropology and Conservation, University of Kent, $U K$

E-mail:sm691@kent.ac.uk

Keywords: postnatal depression; fertility; fitness

Objective. Evolutionary explanations of postnatal depression (PND) have proposed that it is a signal to warn mothers that continued investment in a particular offspring poses a threat to her fitness, and is indicative that she should divert investment away from this infant. Such hypotheses suggest that it is adaptive, and should serve to enhance the reproductive success of women experiencing PND. However, depression is costly to mothers' health and to infant development, and thus seems more likely to be detrimental to fitness and unlikely to help optimize the life history trade-off between quality and quantity of offspring. Until now data on the impact of PND on fertility has been lacking. Methods. An online survey of postmenopausal women $(N=306)$ collected complete reproductive histories, including retrospective measures of PND assessing incidence rate, severity, longevity, and actual diagnosis. Results. PND associated with the first birth led to a reduction in completed fertility, which dropped belowreplacement levels. PND also negatively affected parity progression. Controlling for factors known to influence childbearing, for example kin and paternal support and infant health, results show that experiencing two bouts of PND, increasing PND severity, and PND lasting over six months all reduce the likelihood of a woman progressing to a third birth. Conclusions. PND presents costs to reproductive success in modern low-fertility contexts. While these results represent only the first attempt to bring empirical data to adaptive debates surrounding PND and depression more generally, they nonetheless suggest pathways by which PND is detrimental to fitness by lowering fertility. The below-replacement fertility of women experiencing PND strongly suggests that preventative measures should be a target for those concerned with population ageing and its economic repercussions. 
O-02: Expenditures in public health and biological welfare during Spanish industrialization (1840-1914)

J. Garcia Gomez, ${ }^{1}$ J. Puche Gil, ${ }^{2}$ and E. Trescastro Lopez ${ }^{3}$

${ }^{1}$ University of Almeria, Spain

${ }^{2}$ University of Zaragoza, Spain

${ }^{3}$ University of Alicante, Spain

E-mail: jjgarcia@ua.es

Keywords: public expenditures; sanitary reform; public health; biological welfare; Spanish industrialization; Alcoy

This paper analyzes the effects of the sanitary reform on the biological welfare and the health of the Spanish population during the industrialization process. It focuses on the case of Alcoy, one of the pioneer hubs of the Spanish industrialization. The main sources of information for this study were the municipality budgets of public health and the stature of conscripts. Results show that the initiation of sanitary reform, in the late 19th century, led to improvements not only to the conditions and the salubrity of the city, but also to the citizen's state of health.

Our hypothesis is based on the concept of urban penalty: negative external effects caused by the industrialization and urbanization process led to slight improvements in real wages and caloric intake while the net nutritional status worsened in several stages, leading to declining height and increasing mortality during those decades.

There were market failures during the industrialization process, which required the intervention of the public sector. Only when the public expenditure in the sanitary reform increased both monetary and nonchrematistic indicators of the standard of living started to accelerate their raising.

For as long as the liberal politicians did not increase the public health expenditures and the urban infrastructural assignments on the budgets, Alcoy suffered the initial problems of the urban penalty, with negative effects that were reflected by increasing morbi-mortality and falling stature. Data suggest a positive correlation between active public health politics and biological welfare.

O-03: Why do immune systems harm their bearers? The evolutionary biology of "friendly fire" by antibodies

\section{A. Graham}

Department of Ecology \& Evolutionary Biology, Princeton University, USA

E-mail: algraham@princeton.edu

Keywords: autoimmunity; life history evolution; trade-off; lupus

Antibodies are essential for resistance against infectious diseases such as malaria and influenza and can participate in somatic maintenance. Yet antibodies can also cause debilitating autoimmunity, when they avidly attack host tissue or accrue at such high densities that they damage organs. Data from wild sheep suggest that predisposition to antibody-mediated autoimmunity may, prior to the onset of any autoimmune disease, be associated with enhanced resistance to infectious diseases. Hosts may thus experience a trade-off between susceptibilities to autoimmune and infectious diseases. This trade-off could help to explain the evolutionary maintenance of alleles conferring susceptibility to lupus and other antibodymediated immunopathologies. Might such a trade-off apply in people? I will compare longitudinal data on infectionspecific and self-reactive antibody titers in animal and human cohorts. The data suggest that human beings, like other mammals, may indeed be subject to this trade-off. I will briefly discuss the potential clinical implications of this pattern.

O-04: Misfolding in Ig-like domains: Insights into multidomain protein evolution and implications for amyloid formation

A. Borgia, ${ }^{1}$ M. Borgia, ${ }^{1}$ A. Soranno, ${ }^{1}$ B. Wunderlich, ${ }^{1}$ D. Nettels, ${ }^{1}$ R. Best ${ }^{2}$ and B. Schuler ${ }^{1}$

${ }^{1}$ Department of Biochemistry, University of Zurich, Switzerland

${ }^{2}$ Laboratory of Chemical Physics, National Institute of Diabetes and Digestive and Kidney Diseases, National Institutes of Health, Bethesda, USA

E-mail: a.borgia@bioc.uzh.ch

Keywords: misfolding; amyloid; multidomain proteins evolution; single-molecule FRET

Protein misfolding and the related process of aggregation are key to our understanding of many human diseases, especially those associated with ageing. Proteins comprised of an array of covalently linked repeated domains, often sharing a common fold and similar sequence, are prevalent in the eukaryotic proteome and have a higher propensity to misfold, because of the inherently high concentration of similar protein segments characterizing these protein arrays. Longlived misfolded species then may, in particular cases, seed aggregation, especially at the high cellular protein concentration; folding of these multidomain proteins, therefore, has to be robust and efficient.

Protein domains with all- $\beta$ structure and immunoglobulin-like (Ig-like) topology are widespread in vertebrates, where they perform a wide variety of functions, and are frequently involved in neurodegenerative and nonneuropathic amyloidosis. The molecular basis for the conversion of functional to pathogenic protein forms, however, is largely unclear.

Our previous work on tandem arrays of Ig-like domains from human titin suggested that evolution has acted to prevent interdomain misfolding and aggregation by sequence diversification of neighboring domains. However, we now 
show that misfolding in multidomain proteins is not at all a rare event: more than half of all molecules misfold and populate an unexpectedly wide range of nonnative conformations, regardless of interdomain sequence identity. These nonnative conformations include both domain-swapped misfolded states and species with amyloid-like features which we identified for the first time. Crucially, though, these potentially aggregation-prone misfolded species only form transiently, while stable misfolded states persist exclusively when sequence identity is sufficiently high.

Our results thus shed new light into the evolutionary pressure that shaped the multidomain protein universe, provide a novel link to amyloid formation, and lead to the new hypothesis about the role of sequence divergence between neighboring domains.

O-05: Evolution of the rapidly-mutating salivary agglutinin (DMBT1) gene: A genetic link between subsistence strategy and Crohn's disease

E. Hollox,${ }^{1}$ S. Polley, ${ }^{1}$ S. Louzada, ${ }^{2}$ D. Forni,${ }^{3}$ T. Balaskas, ${ }^{1}$ D. Hains ${ }^{4}$ F. Yang, ${ }^{2}$ N. Prescott,${ }^{5}$ E. Nimmo, ${ }^{6}$ C. Veal, ${ }^{1}$ I. Vind,${ }^{7}$ P. Munkholm,${ }^{7}$ P. Fode,${ }^{7}$ J. Mansfield, ${ }^{8}$ P. Andersen, ${ }^{4}$ J. Satsangi, ${ }^{6}$ C. Mathew, ${ }^{5}$ and M. Sironi ${ }^{3}$

${ }^{1}$ Department of Genetics, University of Leicester, Leicester, $U K$

${ }^{2}$ Wellcome Trust Sanger Institute, Hinxton, UK

${ }^{3}$ Scientific Institute IRCCS E. Medea, Bosisio Parini, Italy

${ }^{4}$ University of Tennessee Health Science Center, Le Bonheur Children's Hospital, Memphis, Tennessee, USA

${ }^{5}$ Department of Medical and Molecular Genetics, King's College London, London, UK

${ }^{6}$ Centre for Genomic and Experimental Medicine, University of Edinburgh, Edinburgh, UK

${ }^{7}$ Microbiology and Infection Control Unit, State Serum Institute, Copenhagen, Denmark

${ }^{8}$ Institute of Genetic Medicine, Newcastle University, Newcastle Upon Tyne, UK

E-mail:ejh33@le.ac.uk

Keywords: copy number variation; subsistence strategy; Crohn's disease; agriculture

The dietary change resulting from the domestication of plant and animal species and development of agriculture at different locations across the world was one of the most significant changes in human evolution. An increase in dietary carbohydrates caused an increase in dental caries following the development of agriculture, mediated by the cariogenic oral bacterium Streptococcus mutans. Salivary agglutinin (SAG, encoded by the DMBT1 gene) is an innate immune receptor glycoprotein that binds a variety of bacteria and viruses, and is present on various mucosa, particularly in the mouth and gut. In particular, SAG mediates attachment of $S$. mutans to hydroxyapatite on the surface of the tooth. We show that multiallelic copy number variation (CNV) within DMBT1 is extensive across all populations and is predicted to result in between 7 to 20 scavenger-receptor cysteine-rich (SRCR) domains within each SAG molecule. Direct observation of de novo mutation in multigeneration families suggests that these CNVs have a very high mutation rate for a protein-coding locus, with a mutation rate of up to $5 \%$ per gamete. We show that DMBT1 CNV is also associated with a history of agriculture across global populations, suggesting that dietary change as a result of agriculture has shaped the pattern of CNV at DMBT1. A deletion variant of DMBT1 has been previously associated with Crohn's disease (CD), and a DMBT1/- knockout mouse has increased levels of colitis induced by dextran sulphate. We performed a metaanalysis of several case-control cohorts typed by us and by the Wellcome Trust Case-Control Consortium (total 2,946 cases, 4,257 controls). One of the CNVs in DMBT1, that is frequent in populations with a carbohydrate-rich diet, is associated with Crohn's disease (odds ratio 1.23 , 95\% CI 1.1-1.38).

O-06: Systemic protection through remote ischemic preconditioning is spread by platelet-dependent signaling in mice

C. Oberkofler, P. Limani, J. Jang, A. Rickenbacher, K. Lehmann, D. Raptis, U. Ungethuem, Y. Yian, K. Grabliauskaite, R. Humar, R. Graf, B. Humar, and P. A. Clavien

Laboratory of the Swiss Hepato-Pancreatico-Biliary (HPB) Center, Department of Surgery, University Hospital Zurich, Zurich, Switzerland

E-mail: clavien@access.uzh.ch

Keywords: remote ischemic preconditioning (RIPC); ischemia reperfusion injury; serotonin; vascular endothelial growth factor

Remote ischemic preconditioning (RIPC), the repetitive transient mechanical obstruction of vessels at a limb remote to the operative site, is a novel strategy to mitigate distant organ injury associated with surgery. In the clinic, RIPC has demonstrated efficacy in protecting various organs against ischemia reperfusion (IR), but a common mechanism underlying the systemic protection has not been identified. Here, we reasoned that protection may rely on adaptive physiological responses toward local stress, as is incurred through RIPC. Standardized mouse models of partial hepatic IR and of RIPC to the femoral vascular bundle were applied. The roles of platelets, peripheral serotonin, and circulating vascular endothelial growth factor (Vegf) were studied in thrombocytopenic mice, Tph1 $1^{-1-}$ mice, 
and through neutralizing antibodies, respectively. Models of interleukin-10 (IL-10) and matrix metalloproteinase-8 (MMP-8) deficiency were used to assess downstream effectors of organ protection. The protection against hepatic IR through RIPC was dependent on platelet-derived serotonin. Downstream of serotonin, systemic protection was spread through upregulation of circulating Vegf. Both RIPC and serotonin-Vegf induced differential gene expression in target organs, with IL-10 and MMP-8 displaying consistent upregulation across all organs investigated. Concerted inhibition of both molecules abolished the protective effects of RIPC. RIPC was able to mitigate pancreatitis, indicating that it can protect beyond ischemic insults. Conclusions. We have identified a platelet serotonin-Vegf-IL-10/MMP-8 axis that mediates the protective effects of RIPC. The systemic action, the conservation of RIPC effects among mice and humans, and the protection beyond ischemic insults suggest that the platelet-dependent axis has evolved as a pre-emptive response to local stress, priming the body against impending harm.

O-07: Variation in the strength and shape of natural selection across three centuries of human civilization

E. Postma and D. Waldvogel

Institute of Evolutionary Biology and Environmental Studies, University of Zurich, Switzerland

E-mail: erik.postma@ieu.uzh.ch

Keywords: natural selection; life-history evolution; fitness; reproductive timing

Although natural selection is ubiquitous in plant and nonhuman animals, the question how selection is acting in human populations remains controversial. Indeed, it has been argued that with the introduction of birth control, and dramatic improvements in medical care, selection has become negligible, and human evolution has come to a halt. However, this idea appears to be at odds with reality, with recent studies showing substantial amounts of variation in fitness, and strong selection on a range of human traits. As of yet we know very little about the generality of these findings, as they are based on a few populations and relatively short-time periods. Here we will present an analysis of a uniquely detailed, longitudinal, and individualbased human dataset, covering a period of three centuries. We will show how the distribution of lifetime reproductive success and its relationship with reproductive timing and lifespan has changed during this period, and explore how these have been shaped by culture, socio-economic status, demography, and the environment. Thereby we will provide one of the most complete investigations of variation in selection in our own species, and a fascinating glimpse into life as we know it.
O-08: Relationship between siderophore availability and virulence in Pseudomonas aeruginosa

M. Weigert, ${ }^{1}$ A. Ross-Gillespie, ${ }^{1}$ S. Brown, ${ }^{2}$ and $\underline{R}$. Kümmerli $^{1}$

${ }^{1}$ Institute of Plant Biology, University of Zurich, Switzerland

${ }^{2}$ Institute of Evolutionary Biology and Centre for Immunity, Infection and Evolution, University of Edinburgh, UK

E-mail: rolf.kuemmerli@uzh.ch

Keywords: Pseudomonas; siderophore; human opportunistic pathogen; evolution of virulence

The ability of bacteria to establish an infection typically relies on the secretion of virulence factors. While the relationship between pathogenicity and virulence factor secretion is well established, little is known about the optimal level at which a virulence factor should be expressed. If production level scales linearly with pathogenicity then virulence factor overproduction may lead to the premature killing of the host, whereas underproduction may compromise the ability of bacteria to establish an infection. Both effects can lead to reduced bacterial fitness, which should select for intermediate levels of virulence factor expression. We tested this hypothesis by experimentally manipulating the availability of pyoverdine - a key virulence factor, produced by Pseudomonas aeruginosa-within a model host, the greater wax moth larvae. We found that pyoverdine availability indeed scaled linearly with host mortality. Furthermore, we showed that within-host bacterial fitness peaked at intermediate levels of pyoverdine availability, supporting our hypothesis that both pyoverdine over- and underavailability compromise bacterial fitness. At the mechanistic level, we found that increased pyoverdine availability lowered pathogen fitness not because it changed bacterial behavior, but rather because it triggered a stronger host immune response. Our findings have consequences for antivirulence therapies that have recently been suggested as alternatives to fight nosocomial multiresistant bacteria. Specifically, we show that nonlinearities between virulence factor expression and pathogen growth, pathogen regulatory adjustments, and host immune responses must be taken into consideration when designing therapies that aim at curbing virulence factor expression.

O-09: A genome-wide exploration of the pleiotropic theory of senescence. Are human disease and senescence the result of natural selection?

J. Rodriguez ${ }^{1}$ and $\underline{\text { A. Navarro }}^{1,2,3,4}$

${ }^{1}$ Institute of Evolutionary Biology, Pompeu Fabra University, Barcelona, Spain

${ }^{2}$ Centre de Regulació Genòmica (CRG), Barcelona, Catalonia, Spain

${ }^{3}$ National Institute for Bioinformatics (INB), Barcelona, Catalonia, Spain 
${ }^{4}$ Institució Catalana de Recerca $i$ Estudis Avançats (ICREA), Catalonia, Spain

E-mail: arcadi.navarro@upf.edu

Keywords: antagonistic pleiotropy disease selection

Human senescence has long been a mystery, with no single universally accepted theory accounting for its ultimate evolutionary causes (if indeed these causes exist). Perhaps the most popular of the evolutionary explanations proposed so far is the pleiotropic theory of senescence, suggested by G. Williams in 1957. This theory states that mutations conferring risk for traits that are damaging for the organism late in life (e.g., after the fertile stage) might be maintained in a population if they are advantageous early in life, when they can result in an increased reproductive success.

In humans, this theory is consistent with evidence coming from certain genes, from specific conditions or from the life-long reproductive patterns of a few animal models. However, an exhaustive assessment of the impact of all these pleiotropic effects in the senescence of our species has not yet been carried out.

Using public metadata from Genome-Wide Association Studies (GWAS), we quantified the global extent and evolutionary implications for our species of the kind of earlylate age antagonistic pleiotropy predicted by the theory. Our preliminary results are twofold. First, they reveal some nontrivial antagonistic pleiotropies that may be relevant to diagnosis and treatment of age-related pathologies. Second, and more interestingly in evolutionary terms, we observe a significant excess of early-late antagonistic pleiotropy in our genomes, some of which present the signature of natural selection.

\section{O-10: Divergence and convergence: Animal models for congenital human erythroid disorders}

A. Makhro and A. Bogdanova

Institute of Veterinary Physiology, University of Zurich, Switzerland

E-mail:makhro@vetphys.uzh.ch

Keywords: erythrocytes; animal models

Human congenital erythroid disorders are caused by various abnormalities such as hemoglobin polymorphisms, increase in membrane permeability to ions, cytoskeletal dissociation and malfunction of enzymes involved in energy metabolism, and redox state. Beta-globin gene variants giving rise to various hemoglobinopathies (sickle cell disease, betathalassemia) are naturally restricted to the regions currently or historically populated by Plasmodium parasite. Protection against malaria in people with sickle cell trait results from the ability of deoxygenated hemoglobin $\mathrm{S}(\mathrm{Hb})$ to polymerize as a substitution of charged Glu to uncharged Val in position 6 which highly reduces its hydrophilicity. Irreversible
$\mathrm{HbS}$ polymerization is triggered by deoxygenation, and markedly facilitated by dehydration that increases mean corpuscular hemoglobin concentration. This strategy to resist infection (protozoa and viruses) is not restricted to humans. Several fish, reptile, and mammalian species are capable of reversible hemoglobin polymerization which is triggered by stress or infection. Among mammals hemoglobin polymerization and sickling were observed in ruminants, genets, domestic cats, and mongooses. Despite the fact that plethora of naturally occurring animal sickle cell disease models have already evolved, several mouse models producing human $\mathrm{HbS}$ were generated to develop therapeutic strategies to treat sickle cell disease patients. The majority of these strategies were proven inefficient in clinical trials. The best of all drugs available for this group of patients, carbamide, is known to boost fetal hemoglobin production in human erythroid precursor cells diluting thereby intracellular $\mathrm{HbS}$. As with all rodents, mice do not express fetal hemoglobin at any point in life, which makes the suitability of mouse models of sickle cell disease questionable. Ruminants, as a result of convergent evolution, do have both fetal hemoglobin and sickle-like hemoglobin polymorphisms. Thus, thinking "out of the rodent box" would be useful for choosing the best animal model for human diseases.

O-11: Extracellular quenching of public goods as an evolutionarily robust treatment against bacterial infection: a case study with Pseudomonas aeruginosa

A. Ross-Gillespie,${ }^{1}$ M. Weigert, ${ }^{1,2}$ S. Brown, ${ }^{3}$ and R. Kümmerli ${ }^{1,2}$

${ }^{1}$ Institute of Plant Biology, University of Zurich, Switzerland

${ }^{2}$ Department of Environmental Microbiology, Swiss Federal Institute of Aquatic Science and Technology, Dübendorf, Switzerland

${ }^{3}$ Institute of Evolutionary Biology, University of Edinburgh, UK

E-mail: adin.ross-gillespie@uzh.ch

Keywords: antibiotic resistance; evolutionarily robust therapy; public goods quenching

Conventional antibiotics select strongly for resistant mutants and, consequently, our existing repertoire of drugs is rapidly losing its clinical efficacy. For more durable solutions, we need virulence-attenuating therapies to which pathogens likely cannot adapt (i.e., resistance will not arise) or where resistant mutants would have no fitness advantage over susceptible types (i.e., resistance will not spread). However, despite much interest and many promising ideas, such "evolutionarily robust" therapies remain rare. We investigated the antibacterial potential of the semimetal gallium against the clinically important opportunistic pathogen, Pseudomonas aeruginosa. Gallium, an ionic mimic of iron, permanently quenches the iron-scavenging 
siderophores secreted and shared among bacteria as public goods, thereby choking off vital iron uptake and imposing a general cost on all siderophore-dependent bacteria in the vicinity. We show that (a) gallium inhibits bacterial growth in vitro in a dose-dependent manner, with intermediate concentrations imposing additional costs by inducing maximal investment in replacement siderophores; (b) gallium significantly attenuates both virulence and bacterial growth in-vivo in an animal model (Galleria mellonella). Crucially, (c) while conventional antibiotic treatments fail to prevent the rapid emergence of resistant mutants in experimentally evolved cultures, gallium treatments retain their efficacy over time. Finally, (d) we further investigate, via competition experiments with engineered mutants, the invasibility potential of several phenotypes we speculated might show resistance to gallium treatment. Collectively, our results suggest that extracellular quenching of bacterial public goods-and in particular, galliummediated siderophore disruption-represents an effective and evolutionarily robust antibacterial strategy.

\section{0-12: The guardians of inherited oncogenic vulnerabili- ties}

T. Tissot, A. Arnal, B. Ujvari, L. Nunney, E. Solary, L. Laplane, F. Bonhomme, M. Vittecoq, A. Tasiemski, F. Renaud, P. Pujol, B. Roche, and F. Thomas

\section{CREEC, Montpellier, France \\ E-mail:tazziotissot@gmail.com}

Keywords: hereditary cancers; purifying selection; genetic drift; oncogene

Similar to seemingly maladaptive genes in general, the persistence of germinally inherited cancer causing mutant alleles in genomes remains a challenging question for evolutionary biologists. In addition to traditional explanations like senescence or antagonistic pleiotropy, we discuss here the paradoxical role of natural defenses to cancer which by preventing neoplasm formation and progression increases organismal fitness, but antagonistically also mask the real effects of cancer causing mutant alleles on fitness and hence protect inherited ones from purging by purifying selection. We provide a mathematical framework to explore these dynamics and show that a likely explanation of why selection pressure favors tumor elimination/control rather than the purifying selection of inherited cancer causing mutant alleles is that, unlike other genetic diseases, most cancers are due to accumulation of somatic mutations. We also argue that ecological contexts experienced by individuals and/or species are crucial considerations because they determine the locally predominant fitness-reducing risks, and hence can aid the prediction of how natural selection will influence cancer outcomes: would it suppress tumor formations or would it purge inherited cancer causing mutant alleles? Acknowledging that natural selection acts as a double-edged sword in cancer development and progression may suggest novel directions to close the loopholes in evolution's strategy for cancer control.

\section{O-13: Peripartum shifts in female fecundity}

\section{Escasa-Dorne}

Department of Anthropology, University of Colorado Colorado Springs, USA

E-mail:mdorne@uccs.edu

Keywords: fertility; reproduction; lactation; postpartum

While medical science traditionally focuses on western populations as the reference point for health and disease, including aspects of reproduction and fertility, our understanding of evolutionary foundations suggests nonwestern populations may provide insight into reproductive outcomes. Anthropologists utilizing an evolutionary theory framework have found utility in applying western biomedical methodology to various populations to identify how evolution has shaped behaviors related to fertility and successful outcomes of reproduction. The varied practices of mating, pregnancy, and the time after giving birth may inform the ways in which we see the navigation of reproductive success via health outcomes. This presentation reviews findings from a dataset from the Philippines concerning shifts in female sexual behavior, partner relationships, and offspring care during the postpartum phase, and discusses comparative literature from nonhuman mammals, cross-cultural postpartum behaviors, and associated endocrinology of the postpartum period. This research adopts a functional, female perspective on this transition, providing a lens into the ways adaptive female strategies play out within socioecological constraints. Despite a massive literature on human and nonhuman animal sex differences in sociosexuality, relatively little has been said about the major effects of reproductive status on both female and sex differences in sociosexuality. Importantly, reconstructions of human evolution suggest that females spent most of their reproductive years pregnant or subject to postpartum lactational amenorrhea (not cycling, in part due to inhibitory effects of lactation), an evolutionary justification for investigating the impacts of pregnancy and postpartum lactation on female postpartum fecundity.

O-14: An evolutionary perspective on the boundary between innate and adaptive immunity

\section{N. S. Greenspan}

Case Western Reserve University, USA

E-mail: nsg@case.edu

Keywords: innate immunity; adaptive immunity; antigen specificity; boundary

Immunologists, other biomedical scientists, and clinicians routinely divide immune mechanisms into "innate" and 
"adaptive." Typical criteria for distinguishing innate from adaptive molecules, cells, and mechanisms include the extent of receptor specificity, the kinetics of the response, and the effect, if any, of prior exposure to a particular stimulus (i.e., is there "memory"?).

While most of the above individuals presumably accept that these physiological systems have originated through evolution, their conceptualization of the relationships between putatively innate and adaptive molecules, cells, and mechanisms is not entirely coherent or informed by evolutionary principles. I will review judiciously selected examples of the phylogenetic, structural, and functional relationships characterizing immunity-related molecules, cells, and mechanisms and note the ways in which evolutionary origins inevitably frustrate the rigid classification criteria typically employed.

Consider B lineage lymphocytes and their progeny, such as plasma cells, which are the sole natural source of antibodies. These cells are prototypical cellular participants in adaptive immunity and are the only cells to employ the pinnacle of adaptive immune mechanisms: somatic hypermutation. Nevertheless, B lymphocytes of the sort that undergo this diversity-generating process and subsequent selection, that is, evolution in the course of an immune response, display numerous receptors (e.g., Toll-like receptors, complement receptors) for molecules regarded as participants in innate immune mechanisms. These same receptors, on other cell types, are regarded as part of innate immunity. So, are these molecules on adaptive immune cells participants of innate immunity or adaptive immunity?

There are many other phenomena that reflect evolutionary opportunism and that defy simplistic categorization schemes not informed by evolution. Insights informed by the evolutionary origins of these immunological molecules, cells, and mechanisms can be harnessed to craft more realistic conceptual frameworks for thinking and communicating about immune responses that better guide further investigation of immune phenomena and intervention in immunity-related diseases.

0-15: Teaching the relevance of evolution for understanding immune recognition and critical thinking in biomedicine

\section{N. S. Greenspan}

Case Western Reserve University, USA

E-mail:nsg@case.edu

Keywords: immune recognition; evolution; logic; critical thinking; teaching

Since 1994, I have taught a roughly alternate-year graduate seminar course. The original course was entitled, "Immunology, Evolution, and Logic," and I was the sole faculty member presiding over every class. There were no invited lecturers.

Beginning in 2009, another faculty member, Derek Abbott, joined me in teaching the course, and the title was revised to "Logical Dissection of Biomedical Investigations." In my portion of the course, I retained an emphasis on the relevance of logic and evolutionary principles to thinking about immune recognition and immune functioning more generally. I focused class sessions on concepts and underlying assumptions critical to experimental investigations as well as on experimental design and data interpretation in articles reporting studies pertaining to immune recognition. Dr. Abbott focused his portion of the course on the practical cognitive skills involved in reviewing papers and grant proposals pertaining primarily to innate immune signaling.

In my portion of the course, I have used questions about the readings to guide students as they grapple with challenging original reports or conceptually-oriented readings on evolution or logic. Another unusual feature pertaining to my sessions was the provision to the students of summaries of class discussions, providing a record of key points to which students could refer in the future.

The course started with a series of questions pertaining to a selection of fundamental scientific facts and more challenging conceptual questions. The students were provided with the same questions at the end of the course. The students' answers provided an opportunity for both faculty and students to assess any gains in knowledge and insight. At the end of the course, we gave the students a survey to provide feedback on many aspects of the class and a list of so-called take-home points from the entire course.

\section{O-16: The origins of ancient and modern tuberculosis}

\section{E. Minnikin}

Institute of Microbiology and Infection, School of Biosciences, University of Birmingham, Birmingham, UK

E-mail:d.e.minnikin@bham.ac.uk

Keywords: tuberculosis; evolution; lipids; biomarkers

In the evolution of tuberculosis, mounting evidence supports a transformation from an environmental organism, such as Mycobacterium kansasii, via diverse ancestors, currently labeled "Mycobacterium canettii", to all the modern biotypes of the Mycobacterium tuberculosis complex. Modern human and animal tuberculosis has emerged through a Pleistocene/Holocene "bottleneck" around 20ka BP (Supply et al. 2013 Nature Genetics 45:172). Ancient DNA and lipid biomarkers have confirmed the oldest human tuberculosis in a $9 \mathrm{ka} \mathrm{BP}$ woman and child from Atlit-Yam, Israel (Hershkovitz et al. 2008 PLoS ONE $3:$ 3426). Evidence is lacking before the Holocene for the presence of tuberculosis in Homo sapiens, but widespread 
dissemination in Pleistocene megafauna is evident. DNA and lipid biomarkers confirmed tuberculosis in a $17 \mathrm{ka}$ BP bison (Lee et al. 2012 PLoS ONE 7:e41923). A good working hypothesis is that Pleistocene megafauna ingested environmental mycobacteria, such as M. kansasii, into their stomach systems where they were subjected to horizontal gene transfer eventually transforming into diverse "smooth" "M. canettii."

Extant " $M$. canettii" strains are included in the broad M. tuberculosis complex and cause the disease tuberculosis. They do have reduced infectivity, however, possibly due to poor aerosol transmissibility. In contrast, modern "rough" M. tuberculosis sensu stricto has preferred transmission in aerosols. The molecular basis for this clear distinction appears to lie in cell-envelope lipid composition. $M$. kansasii and " $M$. canettii" produce relatively hydrophilic lipooligosaccharides and "phenolic" glycolipids in keeping with environmental links. In contrast, modern M. tuberculosis is characterized by hydrophobic sulfoglycolipids that may facilitate aerosol transmission. The apparent expansion of tuberculosis in Homo sapiens, during the Holocene, may be a consequence of hunter-gatherers settling down in stable communities and being confronted with the new highly-contagious tubercle bacillus (Minnikin et al. 2015 Tuberculosis http://dx.doi.org/10.1016/j.tube.2015.02.009, in press; In Tuberculosis, W. Ribón, ed., InTech, Rijeka, Croatia. ISBN 978-953-51-4194-5, in press).

\section{O-17: Bacterial social interactions and their impact on virulence evolution in an opportunistic human pathogen}

\section{E. Granato and R. Kümmerli}

\section{Institute of Plant Biology, University of Zurich, Switzerland} E-mail:egranato@botinst.uzh.ch

Keywords: virulence evolution; Pseudomonas; opportunistic pathogen; virulence factors

Understanding the evolution of virulence in human pathogens is of great interdisciplinary interest. This is particularly true for opportunistic pathogens, such as Pseudomonas aeruginosa, that often cause chronic infections and undergo within-host evolution during disease progression. Here, we use experimental evolution to elucidate the interplay between virulence factor secretion, spatial structure, and the evolution of virulence in the host model-species Caenorhabditis elegans.

Virulence in bacterial infections is typically mediated through secreted metabolites that can be cooperatively shared across the bacterial collective within the host. Although many of these metabolites are essential to establish infections, populations of metabolite producers can potentially be invaded by "cheating" mutants, which no longer contribute but still benefit from the shareable metabolites produced by others. Consequently, conditions selecting for cheats should ultimately reduce virulence.

We tested this prediction by experimentally evolving $P$. aeruginosa in liquid (unstructured) and agar-based (structured) medium in the presence or absence of its host C. elegans. We found that evolved bacteria did not show dramatic changes in the expression of pyoverdine, a secreted metabolite used to scavenge iron from the environment and the host tissue. Conversely, the production of pyocyanin, a secreted toxin important in killing C. elegans, dropped dramatically during evolution. Pyocyanin production was the lowest among bacteria that evolved in liquid medium with the host. This pattern is compatible with the view that nontoxin producing cheats can preferentially invade in an unstructured environment, where secreted metabolites and cells mix readily, and when virulence factor expression is beneficial (i.e., in the presence of hosts). Furthermore, we found that reduced pyocyanin production went along with a small but significant reduction in virulence. Our results highlight that cheating in infections can arise and steer pathogen populations towards lower virulence. This opens possibilities for therapeutic interventions that aim at exploiting cooperator-cheat dynamics.

\section{O-18: Exploring the Neolithic cost: Disease and transi- tion in the Agta, the Filipino foragers}

A. Page

University College London, UK

E-mail: abigail.page.10@ucl.ac.uk

Keywords: hunter-gatherers; health; epidemiological transition; ecology

Major evolutionary medical theories have arisen from the concept of the "original" disease milieu of huntergatherers, distinguished by chronic infestation of parasites. Small and mobile populations escaped the acute infectious diseases prevalent after the Neolithic revolution. However, due to the practical limitations of data collection, critical testing in extant foragers has been overlooked. By creating quantifiable risk indices for the transition between foraging and farming lifestyles, based not only on the disease load but the specific nature of this burden (nutritional, parasitic, viral or bacterial) we can gain insight into humanity's evolutionary past. Furthermore, as the process of transition to agriculture has been labeled as "the worst mistake in human history," compiling evidence is crucial for informing local policies, which actively promote settlement of foragers. If hunter-gatherer health suffers with sedentarization, as theoretically predicted, this research is crucial for foragers' wellbeing.

To examine these themes, we collected biological data from the Agta of Palanan, a heterogeneous Philippine 
population of fisher-foragers. While some Agta reside in jungles and river basins, others live in coastal areas. Within these groups exists a great variance in the degree of settlement, interaction, and distance to farmers. Therefore, the Agta present a perfect testing ground for the effects of sedentarization on health.

My research asks whether the main features of a foraging lifestyle (degree of mobility, wealth, cash labour participation, diet breadth, and camp size) significantly predict disease trends. To test this, we collected hemoglobin levels, white blood cell differentials, fecal analysis, short-term medical histories, and anthropometric data from 420 Agta. With this extensive dataset analyzed using multilevel models, it is possible to examine the impact of camp and individual level variables on health. These results inform our understanding about the disease ecology during human evolution and the selective pressures forming humanity's immune system and behavioral responses.

\section{O-19: Optimal fat storage and the risk of diabetes}

\author{
R. Johnstone, ${ }^{1}$ A. Rodrigues, ${ }^{1}$ and J. Wells ${ }^{2}$ \\ ${ }^{1}$ University of Cambridge, $U K$ \\ ${ }^{2}$ UCL Institute of Child Health, London, UK \\ E-mail:raj1003@hermes.cam.ac.uk
}

Keywords: life history; evolutionary medicine; noncommunicable disease

Storage of energy in the form of fat helps organisms to survive periods of low resource availability. At the same time, excessive fat (especially in relation to lean mass) may carry physiological costs due to increased risk of noncommunicable diseases such as diabetes. Using state-dependent dynamic programming, we determine optimal strategies of investment in fat versus lean tissue during juvenile growth, and in fat versus reproduction during adulthood, when individuals must cope with an uncertain environment and mortality costs of excessive fat storage. We show that strategies adapted to more uncertain environments are selected to store more fat even though this leads to higher diabetes risk. The maladaptive cost of diabetes can thus be interpreted in an adaptive manner as a reflection of the need to prioritize buffering against the risk of starvation over fat-induced mortality. Intriguingly, such strategies also fare poorly in an enriched environment (corresponding, in the human case, to modern conditions) in which resource availability is elevated relative to the environment of adaptation, due to their propensity to store additional resources in the form of fat. Moreover, adult risk of diabetes is predicted to be sensitive to resource availability during juvenile growth, as early starvation leads to lower lean body mass in adulthood, which is associated with relatively higher levels of obesity and fat-related mortality.
O-20: A critical evaluation of evolutionary hypotheses for type 2 diabetes

\section{Watve}

Department of Biological Sciences, Indian Institute of Science Education and Research, Pune, India

E-mail:milind@iiserpune.ac.in

Keywords: thrifty gene; fetal programming; behavioral strategies; game theory

Evolutionary thinking has influenced research and clinical practice in type 2 diabetes mellitus (T2DM). Simultaneously, evolutionary thinking has misled the field from time to time. Appearance of glucose in urine was the first noted symptom of diabetes and insulin was the first successful molecule discovered to regulate plasma glucose. Therefore as a burden of history, although the levels of a large number of molecules are now known to be altered in T2DM, the thinking in this field is still glucose and insulin centered. In 1962, the most influential hypothesis so far about a possible evolutionary origin of obesity and T2DM, namely the thrifty gene hypothesis, was proposed by James Neel. While Neel perceived hyperinsulinemia as the thrifty adaptation and "anti-insulin activity," (now known as insulin resistance) as a compensatory response, later thinking turned the sequence upside down. This change was brought about for the convenience of prevalent theories without any support of evidence. In the 1990s, the observed correlations between birth weight and noncommunicable diseases brought in a major alteration in the concept in the form of predictive adaptive programming. Over the last two decades, the thrifty gene as well as thrifty programming faced serious criticism and a number of alternatives were suggested. The time appears to be ripe today so we can critically evaluate all the alternative hypotheses. I will outline the fundamental expectations from an evolutionary hypothesis of a disease, examine the alternative hypotheses in that framework, and address the question how one can subject them to rigorous testing. We ultimately need a sound theory that is useful at the clinical level in improving prevention, control and possible reversal of T2DM.

O-21: An evolutionary genomics perspective on the impact of HIV coinfection on a Mycobacterium tuberculosis population

D. Brites, E. Wampande, K. Eisenach, H. Boom, M. Joloba, and S. Gagneux

Swiss Tropical and Public Health Institute, University of Basel, Basel, Switzerland

E-mail:d.brites@unibas.ch

Keywords: Mycobacterium tuberculosis; HIV coinfection; evolutionary genetics; whole-genome sequencing 
In the last decades, coinfections by the HIV virus and Mycobacterium tuberculosis have fueled tuberculosis incidence in many countries. Given the importance of CD4+ T-cell responses both in providing protective immunity against tuberculosis as well as drivers of lung pathology and ultimately mediators of transmission, the immunocompromised host environment is likely to affect the life cycle of $M$. tuberculosis. We therefore hypothesized that the immunocompromised host environment affects the fitness of $M$. tuberculosis and tested whether and how HIV/M. tuberculosis coinfections impact the genetic structure of a $M$. tuberculosis population. To test this hypothesis, we analyzed whole-genomes of 180 closely related $M$. tuberculosis strains isolated retrospectively from both HIV infected and uninfected patients over a period of 12 years in Kampala, Uganda. Our study suggests that HIV coinfections have not structured this M. tuberculosis population significantly, but seem to decrease its effective population size, consequently affecting the fate of new mutations in the population. This possibly reflects the fact that $M$. tuberculosis from HIV infected patients is less transmitted if the patients have severe CD4+ T-cell depletion. Supporting the latter, we could show that $M$. tuberculosis from patients with low CD4+ T-cells (less than 250 cell/uL) also tends to have more mutations which are uniquely found in the population (i.e., where not transmitted) than $M$. tuberculosis strains which infect patients with higher CD4+ T-cell numbers. Our results shed light on the fate of mutations of M. tuberculosis isolated from HIV coinfected patients, and suggest that despite the high incidence of $M$. tuberculosis and HIV coinfections in the studied population, M. tuberculosis is not adapting to the host environment induced by HIV infection.

\section{O-22: Characterizing the transcriptome of a transmissi- ble cancer}

B. Uvjari, ${ }^{1,2}$ M. Kowarsky, ${ }^{3}$ E. Wong, ${ }^{2}$ C. Wu, ${ }^{2}$ A. M. $\overline{\text { Pearse, }}{ }^{4}$ R. Taylor, ${ }^{4}$ W. Warren, ${ }^{5}$ K. Belov, ${ }^{2}$ and A. Papenfuss $3,6,7$

${ }^{1}$ Centre for Integrative Ecology, Deakin University, Waurn Ponds, Victoria, Australia

${ }^{2}$ Faculty of Veterinary Sciences, University of Sydney, Camperdown, NSW, Australia

${ }^{3}$ Bioinformatics Division, The Walter and Eliza Hall Institute of Medical Research, Parkville, Victoria, Australia

${ }^{4}$ Devil Facial Tumour Project, Diagnostic Services, Animal Health Laboratory, Department of Primary Industries, Water and Environment, Launceston, Tas, Australia

${ }^{5}$ The Genome Institute, Washington University School of Medicine, St. Louis, USA

${ }^{6}$ Peter MacCallum Cancer Centre, East Melbourne, Victoria, Australia
${ }^{7}$ Sir Peter MacCallum Department of Oncology, University of Melbourne, Melbourne, Victoria, Australia

E-mail: beata.ujvari@deakin.edu.au

Keywords: devil facial tumour disease; transmissible cancer; transcriptome

Devil Facial Tumour Disease (DFTD), is a contagious cancer that threatens the long-term survival of the world's largest marsupial carnivore, the Tasmanian devil (Sarcophilus harrisii). Since the emergence of the disease in the mid 1990s, four karyotypically distinct Devil Facial Tumour (DFT) strains or variants have been described. In the present study, we compare the gene expression profiles of DFTD variants using transcriptome sequencing to investigate whether gene expression differences or expressed mutations could drive phenotypic differences between tumour strains. We found no significant differences in the expression of protein-coding genes between strains. We also report investigations into the possibility of nonsynonymous strain-specific mutations. The transcriptome sequence also provides an important resource of expressed genes, which we have used to identify highly expressed genes with oncogenic and angiogenic potential, including BCL2, NDRG1, and protein kinases, which provide targets for development of therapeutic agents.

\section{O-23: Rates of antibiotic resistance evolution in gonor- rhoea \\ S. Fingerhuth, ${ }^{1,2}$ S. Bonhoeffer, ${ }^{2}$ N. Low, ${ }^{1}$ and C. Althaus ${ }^{1}$ \\ ${ }^{1}$ Institute of Social and Preventive Medicine (ISPM), University of Bern, Switzerland \\ ${ }^{2}$ Institute of Integrative Biology, ETH Zurich, Zurich, Switzerland \\ E-mail: stephanie.fingerhuth@env.ethz.ch}

Keywords: Neisseria gonorrhoeae; gonorrhoea; antibiotic resistance; mathematical modeling

Evolution of antibiotic resistance is a major problem for the treatment of the sexually transmitted infection gonorrhoea. Neisseria gonorrhoeae has developed resistance to all classes of antimicrobials that have been used to treat it, and strains that are resistant to multiple classes of antimicrobials have evolved. Only one antimicrobial (ceftriaxone) can now be used for empirical treatment in many countries. Hence, it is important to understand the dynamics and drivers of resistance evolution.

First, we estimated rates of resistance evolution from surveillance data from the USA and from England and Wales. We found that resistance initially spreads exponentially at rates between 0.2 and 2.4 per year. These rates suggest that the proportion of resistant strains doubles every 3 to 35 months. Interestingly, we found a lower rate of spread in heterosexual men (men having sex with women, MSW) 
compared with men having sex with men (MSM) on average. Second, we developed a dynamic transmission model to reconstruct the observed dynamics of $N$. gonorrhoeae resistance evolution in both MSW and MSM populations. The model allowed us to identify the treatment rate as the driving force behind the spread of resistance. We conclude that there is a trade-off in the treatment rate between providing individual patient care to all those who are infected and keeping the spread of resistance as low as possible.

\section{O-24: Evolutionary and network properties of human disease genes}

N. Spataro, ${ }^{1}$ J. Rodriguez, ${ }^{1}$ A. Navarro, ${ }^{1,2,3,4}$ and E. Bosch ${ }^{1}$

${ }^{1}$ Institute of Evolutionary Biology (CSIC-UPF), Department of Experimental and Health Sciences, Pompeu Fabra University, Barcelona, Catalonia, Spain

${ }^{2}$ Centre de Regulació Genòmica (CRG), Barcelona, Catalonia, Spain

${ }^{3}$ National Institute for Bioinformatics (INB), Barcelona, Catalonia, Spain

${ }^{4}$ Institució Catalana de Recerca $i$ Estudis Avançats (ICREA), Catalonia, Spain

E-mail:nino.spataro@upf.edu

Keywords: human disease; complex diseases; mendelian disorders

How do evolutionary forces shape the genetic architecture of disease genes? Do disease and nondisease genes have different network properties? Do the same evolutionary pressures affect Mendelian and complex human disorder genes? What is the role of coding and regulatory sequences on disease gene evolution? The answers to these questions could shed light on understanding human genetic disorders and help to predict which types of allele variants and allele frequencies contribute to disease risk, with relevant implication on mapping strategies for future genetic studies. We have analyzed and compared sequencing data from the 1,000 Genomes project as well as expression data and the network properties of human Mendelian genes (from the OMIM database), genes associated to complex disorders (from the GWAS catalog database), essential genes (considering the orthologs human gene of mouse essential genes detected by knock-out experiments) and of the remaining human protein coding genes on Ensembl website. Our results show that disease genes have specific evolutionary profiles and protein network properties when compared to nondisease genes. Moreover, those genes that are both causal for Mendelian diseases and risk factors for complex traits of medical relevance tend to show particular protein network and expression features, higher effect sizes and specific evolutionary pressures on their coding and regulatory sequences. The observed pattern on this subset of disease genes suggests that purifying selection could not be the only force shaping the properties of this subgroup and that the classic scenario of mutation-selection balance may be inappropriate for some genes underlying human genetic disorders.

\section{O-25: Fecundity compensation in cancerous flies Drosophila melanogaster}

A. Arnal, C. Jaqueline, A. Casali, B. Roche, C. Moreno, F. Mery, and F. Thomas

CREEC and MIVEGEC, UMR IRD/CNRS/UM Montpellier, France

E-mail:au.arnal@gmail.com

Keywords: cancer; fecundity; Drosophila melanogaster

Hosts often forward their reproductive effort in response to a parasitic infection. These cases of fecundity compensatory have been especially observed when the host's chances of future reproduction decrease with time since the onset of the infection. Because malignancies usually reduce survival, it is expected that hosts that are unable to resist tumorigenesis progression by other means should be favored by selection if they partly compensate for the cancer-induced losses by adjusting their life-history traits. Individuals with early cancer could have evolved to adapt their behavior to maximize both their survival through energy allocation to their immune system and their reproductive success. Despite the potential importance of these plastic responses, little attention has been devoted to explore how cancers influence animal reproduction. In this study, we explore experimentally this novel scientific direction by using genetically modified flies Drosophila melanogaster which develop, after a one-hour heat shock at $37^{\circ} \mathrm{C}$, a colorectal cancer of the anterior gut part. For this, we tested if cancerous drosophila females oviposit earlier than noncancerous ones and if their egg number is different. To ensure that the genetic background does not influence the observed patterns, we also tested the two drosophila strains without the heat shock. Considering the experiment as a whole, noncancerous females laid more than cancerous ones. However, individuals with cancer reached the peak period of oviposition two days before noncancerous ones. No difference between genetic background controls were observed for the number of egg laid or for the date of oviposition peak. It is essential to improve our knowledge of the interactions between oncogenic processes and life history traits knowing that most, if not all, ecosystems on our planet are now polluted by mutagenic substances.

\section{O-26: Darwinian critical care medicine}

\section{J. Morgan}

Yorkshire School of Anesthesia \& Intensive Care, UK E-mail:drjgmorgan@gmail.com

Keywords: allostasis; nutritional immunity; antibiotic modeling; immune brinkmanship 
One specialty puzzlingly slow to appreciate evolutionary perspectives has been critical care medicine. With infection and trauma leading causes of prereproductive death, the force of natural selection on the acute phase response is strong, and evolutionary thinking is thus vital to understand this high stakes phenomenon and capitalize with research to drive improved patient outcomes.

The main contribution of evidence-based critical care has been to demonstrate the benefits of fewer, and more conservative, interventions. We are beginning to appreciate we should be slow to conclude that abnormality in physiological variables necessarily denotes a suboptimal response to the insult and often represents defence mechanisms. The immune brinkmanship theory sees these derangements as a key component of the adaptive acute phase response and, although costly to the host, they are more immediately costly to the pathogen. "Allostasis" describes this adaptive maintenance of this "abnormal" environment.

This presentation provides an overview of the emerging field of Darwinian Critical Care from the perspective of a critical care physician with a strongly evolutionary perspective. It will serve to provide a clinician's perspective on specific, clinical examples of what evolutionary research has brought to critical care with a focus on empirically demonstrated improvements in experimental outcomes leading to changes in practice and better care at the ICU level. The Darwinian perspective is beginning to deliver and it is time these hypotheses are explicitly identified as Darwinian; clinicians and biologists working in these areas recognize each other, and the dawn of Darwinian Critical Care Medicine begins.

Defences: the adaptive significance of the acute phase response; nutritional immunity: the evolutionary answer to the transfusion conundrum; fever: assimilating the HEAT trial. Arms races: optimal utilization of antimicrobials in critical care; the toxic microbiome of the intensive care unit. Conflict: obstetric critical care; the debt to Darwinian medicine.

O-27: Balancing selection at the blood group-related gene B4galnt2 in house mice is associated with tradeoffs between coagulation defects and resistance against gastrointestinal pathogens

M. Vallier, ${ }^{1,2}$ M. Linnenbrink, ${ }^{1}$ P. Rausch,,${ }^{1,2}$ G. Grassl, ${ }^{2}$ and J. F. Baines ${ }^{1,2}$

${ }^{1}$ Max Planck Institute for Evolutionary Biology, Plön, Germany

${ }^{2}$ Institute for Experimental Medicine, Christian-AlbrechtsUniversity of Kiel, Kiel, Germany

E-mail:baines@evolbio.mpg.de

Keywords: balancing selection; intestinal microbiota; inflammation; von Willebrand disease
B4galnt2 is a blood group-related glycosyltransferase whose two murine alleles (driving gastrointestinal and vascular expression) are maintained by balancing selection in house mice and their relatives. The vascular allele induces a defect in coagulation and subsequent bleeding phenotype similar to von Willebrand disease in humans, suggesting that this fitness cost may be offset by other unknown benefits. Interestingly, despite its overall long-term maintenance, the vascular allele is absent in wild mouse populations from Germany and Northeast France, but recently increased in Southwest France as evidenced by a partial selective sweep. This suggests that geographic-dependent selective forces may be operating. Given other examples of blood group-related glycosyltransferase variation in humans, we hypothesize that resistance to pathogen(s) may mediate selection operating on B4galnt2 over space and time. Indeed, experiments in lab mice show that the presence/absence of B4galnt2 expression in the gastrointestinal tract influences the response to Salmonella $s p$. infection. By applying metagenomic approaches in a wild mouse population displaying evidence of recent selection (i.e., a partial selective sweep), we found that B4galnt2 genotype correlates with differences in intestinal inflammation and the presence of candidate pathogens that could drive selection at B4galnt2. We thus suggest the presence of intestinal pathogens to be a potent source of variation in selection and the maintenance of disease-associated alleles.

O-28: Natural variation of DNA methylation profiles along the Italian peninsula: an evolutionary perspective reveals implications for human health and diseases

C. Giuliani, ${ }^{1}$ C. Pirazzini, ${ }^{2,3}$ M. Bacalini, ${ }^{2,3}$ M. Sazzini, ${ }^{1}$ D. Mari, ${ }^{5,6}$ G. Passarino, ${ }^{7}$ C. Franceschi, ${ }^{2,3,4}$ P. Garagnani, ${ }^{2,3}$ and D. Luiselli ${ }^{1}$

${ }^{1}$ Department of Biological, Geological and Environmental Sciences (BiGeA), Laboratory of Molecular Anthropology \& Centre for Genome Biology, University of Bologna, Bologna, Italy

${ }^{2}$ Department of Experimental, Diagnostic and Specialty Medicine (DIMES), University of Bologna, Bologna, Italy

${ }^{3}$ Interdepartmental Center "L. Galvani" (CIG), University of Bologna, Bologna, Italy

${ }^{4}$ IRCCS Institute of Neurological Sciences, Bologna, Italy

${ }^{5}$ Department of Clinical Sciences and Community Health, University of Milan, Milan, Italy

${ }^{6}$ Geriatric Unit, IRCCS Ca' Granda Foundation Maggiore Policlinico Hospital, Milan, Italy

${ }^{7}$ Department of Biology, Ecology and Earth Science, University of Calabria, Rende, Italy

E-mail: cristina.giuliani2@unibo.it

Keywords: DNA methylation changes; epigenetics; Italian population; human adaptation 
Description of population epigenetic variability is a relatively new approach in the study of human microevolution. In fact, DNA methylation changes create phenotypic diversity within an individual and within populations, and are often associated to different susceptibility to diseases. This mechanism constitutes an important reservoir of variability useful for adaptation in response to new stimuli and recent studies have demonstrated that selective pressures are able to shape not only the genetic code, but also DNA methylation profiles (Heyn et al. 2013; Giuliani et al. 2015). Here we depicted the natural variation that occurs in DNA methylation considering individuals who were born and lived in different areas of Italy (i.e., North, Center, and South). The Italian population is indeed very diverse in terms of culture, traditions, history, and genetic background (Boattini et al. 2013; Sarno et al. 2014; Sazzini et al. 2015 in preparation) and constitutes a suitable model to investigate mechanisms of adaptation mediated by epigenetic variations. A whole-genome DNA methylation analysis identified an enrichment of differentially methylated regions (DMRs) located in genes involved in nitrogen compound transport and nitrogen compound metabolic process. Reactive nitrogen has been extensively studied and it is an object of research for environmental issues. Moreover, considering genes that included a DMR, we observed an enrichment of genes involved in response to pathogens, and recent papers suggested that local pathogen diversity constitutes one of the main selective pressures through recent human evolution (Fumagalli et al. 2011).

In conclusion, elucidating natural variation in DNA methylation constitutes a key perspective for investigating the role of epigenetics in determining complex physiological/pathological traits. Changes of DNA methylation profiles could represent both a consequence of the genetic architecture and of the chromatin conformation, and also a more complex mechanism of adaptation of organisms aimed at ensuring individual survival in a certain environment.

O-29: Sharing of adaptive plant knowledge and its implications on resilience in Mbendjele BaYaka huntergatherers of the Congo Basin

G. Salali, N. Chaudhary, J. Thompson, M. Dyble, A. Page, D. Smith, J. Lewis, L. Vinicius, R. Mace, and A. B. Migliano

University College London, UK

E-mail: guldeniz.salali.12@ucl.ac.uk

Keywords: plant knowledge; hunter-gatherers; cultural transmission; BMI

Transmitting knowledge of the use of plants has historically been an essential function of culture and is especially important for health and nutrition in small-scale populations. The wide sharing of adaptive knowledge, which contributes to an individual's chance of survival and reproductive success may explain the resilience of current day populations who do not have access to modern medicine. Here, we study the knowledge and use of 33 plant species in 219 Mbendjele BaYaka hunter-gatherers living in the northern rainforests of the Republic of Congo. The majority of the plant uses concern digestive and respiratory system disorders. This is not surprising given the high prevalence of pathogen-borne diseases in hunter-gatherer populations. We then investigate shared knowledge of plant uses among individuals from different camps and birth regions, and examine whether similarity in sex, age group, and pairwise relatedness affect the extent of shared knowledge. In particular, we ask if the knowledge of medicinal plant use is shared differently (i.e., only within families, or widely among camps) to the knowledge of plant uses related to foraging, social beliefs, food, or material making. Moreover, we examine whether parental medicinal plant knowledge and use have an influence on children's body mass index (BMI). Information on the frequency and the extent of the use of certain plant species, regardless of the camp membership or birth region, may inform us about the adaptive value of the knowledge. For instance, knowledge about the highly toxic or medicinal plants may be shared more widely because of its influence on an individual's fitness. Overall, our findings contribute to a better understanding of the behavioral adaptations which humans have evolved to better cope with pathogens in our environment.

O-30: A review of the "domestication syndrome" in mammals-the role of neural crest in morphological patterns and comparisons to human malformations

M. Sánchez-Villagra, ${ }^{1}$ M. Geiger, ${ }^{1}$ and R. Schneider ${ }^{2}$

${ }^{1}$ Palaeontological Institute and Museum, University of Zurich, Switzerland

${ }^{2}$ Department of Orthopaedic Surgery, University of California, San Francisco, CA, USA

E-mail:m.sanchez@pim.uzh.ch

Keywords: neural crest; domestication; development; mammals

As already emphasized by Darwin, domesticated mammals possess a suite of distinct, heritable traits absent in their wild ancestors: depigmentation of skin and fur, floppy ears, curly tails, short muzzles, small teeth, increased docility, smaller brains, frequent oestrous cycles, and retention of juvenile behavior into adulthood. These traits, the "domestication syndrome," have been proposed to be a result of mild neural crest cell deficits during embryonic development, in turn the result of the selection for tameness during initial stages of domestication. Yet, these interrelationships are not straightforward. Not all domesticated mammals present this suit of traits (e.g., horses). In dogs, there is no evidence for a segregation of the "domestication syndrome" characters 
in breeds which are docile towards humans, as compared to more aggressive and fearful ones, but other factors are involved that explain the remodularization in traits within domesticated species. The role of neural crest is uncontested based on different lines of evidence. The clinical literature on neurocristopathies, some going back to the 1950s, point to the neural crest in the etiology of diverse and complex syndromes that span multiple organ systems and morphological structures in humans. Changes in the activity of tyrosine pathway enzymes within neural crest cells may enable changes in the phenotypic integration of epidermal, nervous, and endocrine tissues. Patterns of domestication mirror some human conditions and are exaggerated versions of what happens with wild forms in being proof points for plasticity and modularity in developmental mechanisms of morphological evolution.

\section{O-31: Changes in mortality in Switzerland, 1870-1910}

\section{$\underline{\text { J. Floris, }},{ }^{1}$ K. Staub, ${ }^{2}$ and U. Woitek ${ }^{1}$}

${ }^{1}$ Department of Economics, University of Zurich, Switzerland

${ }^{2}$ Institute of Evolutionary Medicine, University of Zurich, Switzerland

E-mail: joel.floris@econ.uzh.ch

Keywords: mortality; Gini; trend

An important finding in the relationship between income and longevity is the Preston curve (Preston, 1975): longevity increases with increasing income, but with decreasing rates. Explanations for this phenomenon are improvements in nutrition (e.g., Fogel, 2004), public infrastructure investment (Preston, 1975, 1980, 1996), and better health care, probably more important in the 20th than in the 19th century (e.g., Cutler et al., 2006). Another potential channel is institutional quality, producing increasing longevity and increasing income at the same time (e.g., Anand and Ravallion, 1993; Dasgupta and Weale, 1992).

We analyze these issues with a focus on Switzerland in the period 1870-1910. Because of its regional and cultural diversity, Switzerland is an ideal testing ground for examining the causes of changes in the biological standard of living as reflected by changes in mortality. At the end of the 19th century, Switzerland developed from a relatively poor economy to a country at the top of the European GDPper-capita distribution. At the same time, life expectancy at birth increased from 40.6 years for males and 43.2 years for females in $1876 / 1880$ to 50.65 years/53.89 years in 1910/1919 (Eidgenössisches Statistisches Amt, 1935, pp. 72-75).

We construct cantonal life tables for the period 18701910 based on the federal census. From these life tables, we use life expectancy together with measures for regional economic activity to shed light on the relative importance of the channels mentioned above. As in Peltzman (2009), we use the hypothetical number of survivors at each age to compute mortality Gini coefficients, analyzing the change of social inequality in this dimension.

\section{O-32: Untangling our predisposition to obesity: Adipose tissue as an organ of risk management}

J. Wells

\section{UCL Institute of Child Health, London, UK}

E-mail: Jonathan.Wells@ucl.ac.uk

Keywords: obesity; adaptation; adiposity; life history theory Because obesity is associated with diverse chronic diseases, little attention has been directed to the multiple beneficial functions of adipose tissue. A life history approach sheds new light on the value of energy for adaptation to contrasting environments. Conventionally, life history theory assumes energy must be allocated between competing functions; however adipose tissue allows energy to be stored in the body, and allocated to life history functions at subsequent time-points. Adipose tissue not only provides energy for growth, reproduction, and immune function, but also secretes and receives diverse signaling molecules that coordinate energy allocation between these ends in response to changing ecological conditions. This means that adipose tissue simultaneously regulates life history functions, whilst also further responding to energy stresses as a counter-balancing risk management strategy. The large number of individual alleles associated with adipose tissue illustrates its integration with diverse metabolic pathways. The response of adipose tissue to energy stresses in early life similarly highlights its prioritization. Adiposity therefore represents a key means of phenotypic flexibility within and across generations, enabling coherent life-history strategy in the face of ecological stochasticity. The sensitivity of numerous metabolic pathways to ecological cues makes our species vulnerable to manipulative economic forces, in particular those manifesting through the nutrition transition. Secular trends in body size appear to vary across populations, in part due to contrasting responses of adiposity and height according to the ecological setting. The disease component of obesity may lie not in adipose tissue itself, but in its perturbation with our modern industrialized niche. Efforts to combat obesity would be more effective if they prioritized "external" environmental change rather than attempting to manipulate "internal" biology through pharmaceutical or behavioral means.

O-33: Integrating demography and life history: Toward an inclusive fitness theory of adiposity

\section{A. Rodrigues}

Department of Zoology, University of Cambridge, UK E-mail:ammr3@cam.ac.uk 
Keywords: adaptation; ecology; kin selection; resource allocation

Organisms have to allocate scarce resources among competing fitness components. Understanding optimal resource allocation during the lifetime of individuals in a demographic context is crucial to the understanding of a vast array of diseases that have rapidly increased in prevalence among human populations. However, theoretical models that take into account both the demography and the life history of individuals have been relatively neglected. In a demographically explicit context, the resource-allocation strategies of individuals affect not only their own direct relative fitness, but also the relative fitness of their relatives. In this study I develop a general kin selection model in a demographic setting, in which the life history of individuals is explicitly described. Individuals are subject to trade-offs among fitness components, and as they age they need to decide whether to allocate resources to current reproduction or to invest into current survival or into future fitness components. I find that limited dispersal of offspring greatly influences the selective pressures acting on a mother's life history traits. Specifically, limited dispersal increases competition for local resources among siblings and between mothers and offspring. As competition among relatives increases, younger mothers are selected to invest more into survival than reproduction, whereas older mothers are selected to invest relatively more into current reproduction. If individuals suffer from food shortages, in an otherwise affluent environment, relatively more resources are diverted into survival, especially at younger ages. In sum, local competition for scarce resources among close relative can greatly affect the patterns of age-specific investment into survival, and therefore it may be a powerful force mediating the evolution of adiposity.

\section{O-34: Chronic pelvic pain-evidence of evolutionary explanations}

\section{J. Jarrell}

University of Calgary, Canada

E-mail: john.jarrell@albertahealthservices.ca

Keywords: pain maladaptation

Pain can modify the nervous system. Pain sensitization occurs when a subject becomes more sensitive and has more pain with less provocation. Chronic pelvic pain is a very common affliction, usually a consequence of recurrent, persistent visceral disease. When the pain is severe and of long duration, pain sensitization emerges. The specific object of this project was to observe pain sensitization from an evolutionary perspective. Methods. one hundred and seventy-one women with chronic pain for more than three months agreed to provide clinical history and physical findings into a database for analysis. Data from multiple sources were collected to estimate the pattern of increasing menstrual function from hunter-gatherers to present. Results. The average woman is now estimated to have more than 400 menstrual cycles. Severe pain with menstruation occurs with approximately 20\%. Women in the chronic pain database had a higher rate of $78 \%$. With sensitization, there is even greater pain experience specifically dysmenorrhea $(88.2 \%$ vs. $59.6 \%, P<.001)$ and continuous pain $(72.0 \%$ vs. $36.7 \% P<.001)$. Pregnancy loss prior to viability is greater among women with pain sensitization compared to those without sensitization (72.9\% vs. $29.7 \%, P=.011)$. Comment. There are several evolutionary considerations at work in this complex disorder. First, the shift to contraception and increasing menstrual function increases the risks of dysmenorrhea and subsequently chronic pelvic pain and ultimately pain sensitization - a truly maladaptive state due to trade-offs. A similar process may be affecting the greater rates of other common pain conditions in women. Secondly, the increased rate of pregnancy loss prior to viability suggests an extreme form of developmental origin of disease in which women who are severely challenged from a biological, social, and emotional perspective, suffer pregnancy loss to prevent future illness in the late term fetus, neonate, child or adult.

\section{O-35: Sociality and health: Examining the links between inequality, social networks, biosocial stress, and health among Tsimane forager-horticulturalists}

A. Jaeggi, ${ }^{1}$ A. Blackwell, ${ }^{1}$ J. Stieglitz, ${ }^{2}$ C. von Rueden, ${ }^{3}$ B. Beheim, ${ }^{4}$ M. Emery Thompson, ${ }^{4}$ B. Trumble, ${ }^{1}$ H. Kaplan, ${ }^{4}$ and M. Gurven ${ }^{1}$

${ }^{1}$ Department of Anthropology, University of California Santa Barbara, USA

${ }^{2}$ Institute for Advanced Studies, Toulouse, France

${ }^{3}$ Jepson School of Leadership Studies, University of Richmond, USA

${ }^{4}$ Department of Anthropology, University of New Mexico, USA

E-mail: ajaeggi@anth.ucsb.edu

Keywords: inequality; biosocial stress; depression; lifehistory theory

Small-scale foraging societies are characterized by an absence of wealth inequalities and high interdependence; sociality traits that are associated with better health outcomes and psychological well-being in modern societies. To better understand the links between sociality and health we examined the effect of incipient wealth inequality and social network size on cortisol levels, social conflicts, reproduction, immune function, and health outcomes among Tsimane forager-horticulturalists $(N=920)$. Wealth inequality in Tsimane communities $(N=16$, Gini coefficients $=0.18$ $0.44)$ spanned the entire range of industrialized societies, 
making this an ideal study population. We hypothesized that Tsimane in more unequal communities adopt faster life history strategies, shifting investment to mating competition and reproduction and away from immune function, thus resulting in worse health outcomes. Results indicate a strong independent effect of inequality on male social strategies and network size, with increasing conflicts, depression, and cortisol levels in unequal communities, yet little to no effect of inequality on pace of reproduction, immune function, or health outcomes. These divergent results might partly be explained by different sensitive periods for life-history trade-offs combined with the relatively recent advent of wealth inequalities among the Tsimane. Alternatively, inequality in modern societies may affect health primarily due to chronic disease such as diabetes or cardiovascular disease, which is near absent among Tsimane. Ongoing longitudinal research will shed further light on these possibilities.

\section{O-36: On the role of adaptation in heritable diseases and Phylomedicine}

\section{$\underline{\text { S. Kumar }}$}

Institute for Genomics and Evolutionary Medicine (iGEM), Temple University, USA

E-mail: s.kumar@temple.edu

Keywords: selection; disease; medicine; prediction

Nature has been the greatest experimenter on Earth for millennia. New mutations continuously arise in personal genomes, with their fate in populations and species primarily determined by the actions of purifying selection, genetic drift, and positive selection. Comparative sequence analysis at individual, population, and species levels yield a record of their outcomes in the form of patterns of conservation and divergence. These molecular evolutionary patterns and their underlying causes are now the foundation of approaches to forecast potentially disruptive mutations found in our personal genomes. Predictive evolutionary techniques and the associated fundamental research investigations are encompassed by Phylomedicine, which is becoming a key discipline at the intersection of molecular evolution, genomics, and biomedicine. I will present results from our recent work in Phylomedicine and the role of positive (natural) selection in genetic diseases.

\section{O-37: Why do humans need large intervertebral discs?}

\section{Hershkovitz, D. Stein, and H. May}

Department of Anatomy and Anthropology, The Dan David Center for Human Evolution and Biohistory Research, the Steinhardt Museum of Natural History and National Research Center, Sackler Faculty of Medicine, Tel Aviv University, Israel

E-mail: anatom2@post.tau.ac.il
Keywords: intervertebral disc; spine; human evolution; erect posture

The unique bipedal stance humans have adopted has generated vast anatomical adaptations throughout the entire body. The human spine, like other anatomical structures, had to adapt to modified strains and loads, applied from directions at new angles. These novel changes have influenced the spine with its complex composition of bones, joints, ligaments, and cartilage. One of the changes that has received considerable attention is the intervertebral disc, mainly due to its accompanying pathologies. Nonetheless, the evolutionary story of the intervertebral discs has never been examined through the framework of evolutionary medicine.

In the current paper, we will follow the evolution of the intervertebral disc and show that much of the change seen in its size and structure over the course of time is probably not associated, contrary to the common notion, with our unique erect posture.

\section{O-38: Aging changes to the brain and skull}

H. May

Department of Anatomy and Anthropology, The Dan David Center for Human Evolution and Biohistory, the Steinhardt Museum of Natural History and National Research Center Sackler Faculty of Medicine, Tel Aviv University, Israel E-mail: mayhila@post.tau.ac.il

Keywords: aging of the brain; hyperostosis frontalis interna; intracranial volume; cranial bone thickness

It has been widely discovered that the volume of the brain and/or its weight declines with age at a rate of approximately $5 \%$ per decade after the age of 40 , with the actual rate of decline possibly increasing with age, particularly over the age of 70 years. The aging process of the brain volume and weight follows HFI development with age. The pathophysiology of these two parallel processes is still an enigma. However, common characteristics may offer the key; for example, brain changes do not occur to the same extent in all brain regions, for example, the prefrontal cortex is the most affected, whereas the occipital cortex is the least affected. These findings correspond with the cognitive changes observed in ageing. In the current study, I will sketch the parallelism between brain atrophy and cranial bone thickening in different regions and attempt to reveal, in the light of evolutionary medicine principals, why male and female skulls react differently to the shrinking brain.

O-39: Pulmonary artery pressure and arterial oxygen saturation in general population of high altitude living between 3,500 and 4,300 m. Meta-analysis

$\underline{\text { R. Soria }},{ }^{1}$ M. Egger, ${ }^{2}$ U. Scherrer, ${ }^{1,3}$ B. Bender, ${ }^{2,4}$ and S. Rimoldi $^{1}$ 
${ }^{1}$ Department of Cardiology and Clinical Research, Inselspital Hospital, University of Bern, Switzerland

${ }^{2}$ Institute of Social and Preventive Medicine, University of Bern, Switzerland

${ }^{3}$ Department of Biology, Faculty of Science, University of Tarapacá, Arica, Chile

${ }^{4}$ Evolutionary Studies Institute, University of the Witwatersrand, Johannesburg, South Africa

E-mail: rodrigo.soriamaldonado@insel.ch

Keywords: high altitude; systolic pulmonary artery pressure; low altitude

Background. There are millions of people living at high altitude (HA). Pulmonary artery pressure (PAP) and pulmonary vascular resistance represent an important feature. Thus, while there is agreement that pulmonary vascular maladaptation to HA may have important consequences on long-term health and diseases in HA dwellers, there is no consensus on normal PAP in general population at HA. The aim of the study was to provide an estimate of normal PAP in general population at HA. Methods. We meta-analyzed all published data during the past decade on echocardiographic estimations of PAP in general population at HA. Original articles that reported estimations of systolic PAP by Doppler echocardiography in healthy participants living at $\mathrm{HA}(>2,500 \mathrm{~m}$ above sea level) or at low altitude $<1,500 \mathrm{~m}$ above sea level were included. Results. We found in high altitude general population a mean systolic PAP at rest of $25.28 \mathrm{~mm} \mathrm{Hg}$ [95\% CI 23.71, 26.84], whereas at low altitude mean systolic PAP was significantly lower, $18.16 \mathrm{~mm} \mathrm{Hg}$ ([95\% CI 16.57, 19.74], $P<.001$ vs. HA). Mean arterial oxygen saturation $\left(\mathrm{SaO}_{2}\right)$ at rest at high altitude was $90.77 \%$ [95\% CI 89.50, 92.05], while $\mathrm{SaO}_{2}$ at rest at low altitude was significantly higher (98.08\% [95\% CI 97.70, 98.46], $P<.001$ vs. high altitude). Conclusion. This is the first meta-analysis on PAP and $\mathrm{SaO}_{2}$ in general population living at HA. These results will contribute to the understanding of physiological adaptations to high altitudes in humans. Several genetic analyses showed evidence for a recent evolutionary adaptation to HA in Andean, Tibetan, and Ethiopian populations, affecting different physiological pathways. As in our sample, most HA dwellers are of one of these populations and most low altitude dwellers are Caucasians, we cannot distinguish between genetic differences and physiological adaptations (acclimatization).

O-40: Redefining disease: Importance of an evolutionary cross-species perspective

\section{$\underline{\text { E. Uhl }}$}

Department of Pathology, College of Veterinary Medicine, University of Georgia, USA

E-mail:euhl@uga.edu
Keywords: disease definitions; cross-species; animal models; disease phenotypes

Throughout history, disease definitions have changed with advances in technology. Current disease classifications are largely based upon observable findings including: clinical signs and symptoms, pathology, and response to treatment. Recent advances now allow genotypic and bioinformatic information to be incorporated into disease definitions. This shifting paradigm is leading to a more evolution-based approach to medicine that will enhance our understanding of what constitutes "health" and "disease." Given that few diseases are truly unique to humans, and that genome sequencing has revealed substantially greater genetic homology between man and animals than was expected, a logical approach is to devise an integrated disease classification system that incorporates both animal and human diseases. However, for most of the 20th century, medicine, and thus the study of disease, has been missing an evolutionary perspective. The consequences of this in terms of defining disease need to be addressed, as without evolution there is no context to assess the importance of mechanistic or phenotypic variations in manifestations of disease, either across species or between affected individuals of the same species. Thus animal models of human disease have been based almost entirely upon their similarities to human disease, most commonly to tissue lesions. As a result, the genetic and molecular complexity underlying many disease phenotypes has often been underestimated until revealed by failure of the models to predict human responses. Adaptation of a disease classification system that uses genetic information applied in an evolutionary context across species will bring our disease definitions closer to the entities being defined by gene expression patterns and allow environmental influences on disease phenotypes to be better characterized. This will improve understanding of and communication about diseases, particularly "complex" diseases, in ways that will improve diagnoses, yield new targets, and translate to novel therapeutic and preventive interventions for human and veterinary medicine.

\section{O-41: Failing hearts and failed definitions}

\section{N. J. Warner}

Warner Consulting, USA

E-mail:jean@cybercomp.net

Keywords: heart failure; cardiomyopathy; disease terminology

Heart failure is a major cause of morbidity and mortality in developed countries and the incidence and prevalence is increasing worldwide. Differences in susceptibility among individuals and across species are not well understood and 
the current systems of classification, for example dilated, restrictive, and constrictive cardiomyopathy, are not useful for predicting prognosis and treatment effects. Confusion and controversy is such that in the latest (2015) version of Braunwald's Heart Disease, Falk and Hershberger begin the chapter on cardiomyopathies by stating, "There is, at present, no universal definition of cardiomyopathy." Currently, patients with heart failure are simply assigned to two broad groups: heart failure with reduced ejection fraction (HFrEF) and heart failure with preserved ejection fraction (HFpEF). Treatment guidelines for these two diagnoses are the standard of care. Despite recent advances in therapy, the mortality and morbidity in both HFrEF and $\mathrm{HFpEF}$ remain high. Individual patients do not respond predictably and differences in response exist between racial and gender subgroups, implying that evolutionary factors are involved. Advances in genome-based technologies and animal models, including characterization of spontaneous dilated cardiomyopathy (DCM) in different species, are improving our knowledge of heart failure and identifying new pathways and targets for potential therapeutics. To date more than 30 genes in diverse pathways have been identified as resulting in a DCM phenotype in humans. Animal models allow the exploration of the effects of genetic, genomic, and environmental influences on the development and progression of heart failure phenotypes using techniques that are not possible in humans. Understanding the evolutionary basis for why the features of heart disease vary across species as well as the mechanisms behind cardiac adaptations, like those in hibernating grizzly bears, will further understanding of cardiac function and help provide more useful definitions of human disease.

\section{O-42: Evidence of ancient diet related to the emergence of native American type II diabetes}

\section{K. Reinhard}

School of Natural Resources, University of Nebraska, Lincoln, USA

\section{E-mail: kreinhard1@mac.com}

Keywords: coprolite; paleonutrition; NIDDM; Arizona

The elevated prevalence of Non-Insulin-Dependent Diabetes Mellitus (NIDDM) in Native Americans of the Southwest has been explained by several authors in terms of a dietary change from pre-industrial, traditional foods to modern foods. Ancient human physiology became adapted to traditional foods and recently became deleterious during the process of modernization. Although several versions of this hypothesis exist, they all relate to the rise in modern NIDDM with change from prehistoric subsistence practices to modern dietary practices. This is especially true for the Southwestern desert tribes of Arizona and New Mexico.
Coprolites are desiccated feces that are excavated from ancient sites. Coprolite analysts have been recovering the sort of data needed by diabetes researchers to explore the prehistoric dietary foundations for NIDDM. Diabetes researchers have missed these studies that are essential in understanding ancient diet. We are taking this opportunity to show how coprolite analysis of diet provides data relevant to understanding diabetes. Our case example comes from Antelope Cave, Mojave County, Arizona. There was a high reliance on fiber-rich plant foods with low glycemic indices. However, these were not just famine foods as suggested by the original "thrifty gene" hypothesis. These were the food eaten on a day-by-day basis during all seasons, in both feast and famine.

\section{0-43: Opposite-sex facial attractiveness: Preference of self-similar or dissimilar features?}

\section{J. Tutkuviene}

Department of Anatomy, Histology and Anthropology, Faculty of Medicine, Vilnius University, Lithuania

\section{E-mail: janina.tutkuviene@mf.vu.lt}

Keywords: facial attractiveness; preference; self-similar; dissimilar

Assortative mating theory emphasizes the preference of phenotypic similarity in the judgment of oppositesex attractiveness. It is assumed that facial averageness is related to mate quality, genetic diversity, phenotypic fitness and immunocompetence, while nonaverage facial features reflect homozygous (less beneficial for general health status) genotypes. However, judgments on oppositesex attractiveness in relation to detail morphology of the assessor's facial features are not fully discovered. The purpose of the present study was to investigate how people with different facial features evaluate attractiveness of opposite-sex faces with close similarity or far-off dissimilarity. The study consisted of two stages. Firstly, 62 young (18-25y old) males and females rated the attractiveness of different facial features (forehead, eyes, nose, cheeks, lips, chin, etc.) of the opposite-sex looking to the standardized pictures from special atlases (Assman et al., 2007; Ohlrogge et al., 2009): males and females with the average facial features generally ranked as the most attractive of the average size facial features of the opposite gender; males and females with the petit facial features ranked as the most attractive bigger than average features of the opposite-sex, and contrariwise. In the next stage, 124 young males and females rated attractiveness of the opposite-sex persons looking at the standardized photographs (each assessor rated 100 photos of the opposite sex faces): there was no significant correlation between ratings of males attractiveness and their facial 
averageness, in addition, females often rated males with dissimilar corresponding facial features as mostly attractive; males with petit facial features gave the highest scores for all females (also for those with bigger than average facial features), while males with average facial features particularly rigorously rated attractiveness of all females. In conclusion, marginal in size and shape facial features might be attractive for the individuals with dissimilar corresponding facial features.

\section{O-44: An evolutionary perspective on human body mass}

\section{B. M. Auerbach}

The University of Tennessee, Knoxville, USA

E-mail:auerbach@utk.edu

Keywords: evolutionary model; mechanical method; morphometric method

Researchers use body mass in past human populations to assess health, to standardize the effects of activity on the skeleton, and, most often, to examine evolutionary correlates, such as ranging behavior, as well as the presumptive effects of natural selection. In all of these cases, body mass is most often estimated using one of two methods: a mechanical method based on linear regressions calculating mass from the femoral head; and a linear morphometric estimation predicated on a cylindrical geometric model for human body shape, where stature is the height and bi-iliac breadth is the diameter. Both of these methods inherently presume that the relationship of body mass with these three dimensions-femoral head diameter, body breadth, and stature (or its proxy) -is consistent among all human groups. This implicitly means that mass must coevolve with these three dimensions, such that selection on body mass will cause covariant changes in femoral head size, and in body breadth and stature.

This study examines the evolutionary relationships of these dimensions with body mass in two cadaveric samples with documented body masses, the Hamann-Todd Collection and the Dart Collection $(n=200)$. Despite known errors in recorded body mass, these samples provide the best current scenario for this study. Based on the work of Hansen and Houle (2008), evolvability and autonomy were calculated for all dimensions. Results indicate that the femoral head does not evolutionarily covary with body mass, and that femoral length has an effect on its autonomy to respond to evolutionary changes in body mass. Thus, these dimensions exhibit independent evolution; their use as universal estimators in estimating body mass among all humans, as well as their ancestors, is questionable. These results argue that, in estimating body mass from skeletal dimensions of archeological and paleontological human remains, researchers must embrace an evolutionaryinformed approach.
O-45: Teaching evolutionary medicine to undergraduates: Undermining ideological apathy and antipathy with scientific literacy and applicability

A. Foley and D. Collins Cook

Indiana University South Bend and Indiana University Bloomington, USA

E-mail: ajfoley@indiana.edu

Keywords: pedagogy; education; evolution; culture

Evolution education faces significant cultural and structural challenges in America. The general public view evolution as a religious/ideological issue rather than a biological process. As such, engagement in evolution is primarily modulated by religious sentiment and socialization. A significant minority of the American population report that they are skeptical of evolution and even more feel "unsure" about their opinions towards biological change, highlighting not only the ideological nature of the issue, but also the dearth of exposure to evolutionary theory in primary and secondary education. General misunderstanding of evolutionary principles creates barriers to postsecondary biological and medical education, as does a general apathy towards evolutionary education (Hill, 2014). Paradoxically, information on human health and medical science is increasingly more accessible to, and accepted by, the general public.

Evolutionary Medicine lends a practical focus to the teaching of evolutionary theory and thus can be used to engage undergraduate students in the applicability and acceptability of evolution research. In this paper, we will share our experience in teaching evolutionary/"Darwinian" medicine on two campuses within a large, public, Midwestern university system. While the two campuses differ in size and student demographics, in both environments, this course is taught through the Anthropology department, drawing students from many different fields, including nursing and pre-med. We suggest that evolutionary medicine education not only allows students to conceptualize evolutionary theory as a tangible mechanism, but also empowers them to think and talk about health problems in a more comprehensive way. This paper will highlight key pedagogical methods that encourage students to become familiar with evolutionary concepts and to develop their scientific literacy within the context of evolutionary medicine.

O-46: TB, or not TB, that is the question! Molecular DNA-based detection of tuberculosis in ancient human remains

F. Maixner, ${ }^{1}$ A. Posa,${ }^{2,3}$ S. Loesch, ${ }^{4}$ D. Tuarev, ${ }^{5}$ T. Rattei, ${ }^{5}$ E. Molnar, ${ }^{2}$ G. Palfi, ${ }^{2}$ and A. Zink ${ }^{1}$

${ }^{1}$ Institute for Mummies and the Iceman, EURAC Research, Bolzano, Italy

${ }^{2}$ Department of Biological Anthropology, University of Szeged, Szeged, Hungary 
${ }^{3}$ Research Centre for the Humanities, Institute of Archaeology, Hungarian Academy of Sciences, Budapest, Hungary

${ }^{4}$ Department of Physical Anthropology, Institute of Forensic Medicine, University of Bern, Bern, Switzerland

${ }^{5}$ CUBE-Division of Computational Systems Biology, Department of Microbiology and Ecosystem Science, University of Vienna, Vienna, Austria

E-mail: frank.maixner@eurac.edu

Keywords: tuberculosis; Mycobacterium tuberculosis; ancient DNA; molecular diagnostics

Many ancient diseases such as tuberculosis (TB), leprosy or cholera are re-emerging and threatening large populations in various countries. Despite the significant efforts to reduce the spread of these diseases, recent approaches often fail due to the lack of knowledge about the history and evolution of these pathogens. Paleomicrobiology offers the unique opportunity to bring together different scientific expertise such as paleopathology, microbiology, and molecular biology to illuminate the evolutionary history of infectious diseases.

Since the advent of ancient DNA (aDNA) studies more than 30 years ago, M. tuberculosis was the first and so far most comprehensively studied ancient pathogen in the emerging field of paleomicrobiology. The dissemination via the blood-stream during primary TB involves the skeletal system leading to characteristic macromorphological alterations, such as a severe angular kyphosis. This advanced stage of tuberculosis can be detected in skeletal remains and serves as an ideal starting point for molecular investigations.

Most aDNA studies so far assessed the presence of TB DNA using a PCR-based assay targeting the multicopy IS6110 region. More recently, high-throughput sequencing was introduced in the field of paleomicrobiology enabling researchers to reconstruct full ancient TB genomes. This development is inevitably linked to the constant adaptation of modern molecular methodologies to the needs of ancient DNA (aDNA). Particular challenging to genetic paleomicrobiology is the high abundance of environmental DNA from all sorts of microorganisms in DNA extracts of ancient bone and soft tissue samples. In this presentation, we will introduce our paleopathological and molecular findings of TB cases from various geographic regions, dating from the Neolithic to the Middle Ages, and discuss the challenges and opportunities of molecular TB detection in ancient human remains of different periods.

\section{0-47: An evolutionary explanation for the presence of cancer nonstem cells}

K. Sprouffske, A. Aktipis, J. Radich, M. Carroll, A. Nedelcu, and C. Maley

Institute of Evolutionary Biology and Environmental Studies, University of Zurich, Switzerland

E-mail: kathleen.sprouffske@ieu.uzh.ch
Keywords: evolution; cancer; stem cells

Contrary to conventional views that assume all cells in a neoplasm can propagate the tumor, the cancer stem cell hypothesis posits that only a fraction of the cells (the cancer stem cells) can act as tumor-propagating cells, while most of the tumor is composed of cells with limited replication potential. Here, we offer an evolutionary approach to this controversy. We used several evolutionary, computational models to investigate cancer cell dynamics and conditions consistent with the stem cell hypothesis. Our models predict that if selection acts at the cell level, neoplasms should be primarily comprised of cancer stem cells, in contrast to experimental data indicating that neoplasms contain large fractions of cancer nonstem cells. We explore several solutions explaining the paradoxical existence of cancer nonstem cells in neoplasms, including the possibility that selection acts at the level of multicellular proliferative units.

\section{O-48: Darwinian prescriptions-A possible research agenda in evolutionary medicine}

M. Grassberger and M. Pilz

Department of Anthropology, Faculty of Life Sciences, University of Vienna, Austria

E-mail: martin.grassberger@univie.ac.at

Keywords: biotherapy; fly larvae; helminths; bacteriophages

The clinical use of living organisms for the treatment of human diseases-a subject recently termed "biotherapy"includes some unusual therapeutic modalities, which derive their documented efficacy from evolutionary processes. Maggot therapy, for example, is the therapeutic application of blowfly larvae, which evolved to live in the most hostile environments teeming with highly pathogenic bacteria. Some evolved into facultative parasites, feeding on the necrotic tissues of wounds, an evolutionary adaptation that is successfully used to remove dead tissue and combat infection in nonhealing wounds.

Helminth therapy - the therapeutic introduction of parasitic helminths into the body—provides another intriguing example of a therapeutic modality based on host-parasite evolution. In order to survive our immune system, many parasitic worms developed mechanisms to modulate their hosts' normal immune defense mechanisms. With the decline of parasitic infections in western countries over the past century, autoimmune diseases rose significantly. With exposure to helminths again, many patients have experienced significant remission of their symptoms. We are just beginning to understand why.

Phage therapy, the use of bacteriophages to treat infections, is perhaps the most promising evolution-based therapeutic modality in the current major health crisis triggered by the rapid increase of antibiotic resistant bacteria. The 
body of scientific publications concerning biotherapy has grown astonishingly large, especially during the past several decades and it has developed into a rapidly advancing multidisciplinary field of medicine.

Interestingly, however, the evolutionary medicine community, focusing predominantly on the evolutionary origins of diseases, so far did not include these promising topics into their research agenda, reflected in the complete absence of related chapters in major textbooks in this field. Employing evolutionary insights to the understanding and future application of promising efficacious therapeutic agents should become an integral part of interdisciplinary evolutionary medicine research. This presentation aims to trigger this exciting journey.

O-49: Public works loans, social intervention and mortality change in England and Wales, 1850-1914

$\underline{\text { B. Harris }}^{1}$ and A. Hinde ${ }^{2}$

${ }^{1}$ University of Strathclyde, UK

${ }^{2}$ University of Southampton, $U K$

E-mail: bernard.harris@strath.ac.uk

Keywords: public works loans; mortality; enteric fever; diarrhoeal diseases

In an earlier paper (Harris and Hinde 2014), we examined the influence of public works loans on changes in mortality in England and Wales during the late 19th and early 20th centuries. Our analysis suggested that these loans had very little effect on the decline of mortality from enteric fever and diarrhoeal diseases in thirteen "high-performing" districts, and that they only had a limited effect on changes in mortality after that date in 32 areas which borrowed relatively large sums of money at different points in time between 1871 and 1900. The analysis of Harris and Hinde (2014) was limited to the loans which were sanctioned by the Local Government Board (LGB) in England and Wales, and which were taken out by urban sanitary authorities under the Public Health and Local and Confirmation Acts. As the LGB was only set up in 1871 , any loans which were contracted before that date were excluded. Since we know that mortality from typhus and enteric fever declined substantially in most of England and Wales between the 1860s and the 1870 s, we cannot rule out the possibility that investment in public infrastructure (notably water supply and sanitation) before 1871 was an important factor in the rate and timing of mortality decline from causes which might have been expected to respond to this investment.

This issue is important in the context of the debate about the causes of mortality decline in the second half of the nineteenth century. Szreter (1988, pp. 8 and 12), for example, argued that the decline in mortality from typhoid took place after 1870 , as it was not until "the last
30 years of the nineteenth century [that]... most of the significant improvements and works of construction and concrete applications of preventive health measures went forward" (Szreter 1988, p. 22). He characterized the earlier period from the 1830 s to the 1870 s as a "heroic age" in which public health entered the political consciousness, investigations were mounted, legislation was enacted, and Edwin Chadwick "formulated... his ambitious programme" (Szreter 1988, p. 21) but rather little was actually achieved on the ground.

Yet it is clear from the historical record that loans for the purposes of investment in public works were being sanctioned from at least 1850 . These loans were approved by the General Boards of Health and in the early 1850s many of the documents sanctioning them were signed by Chadwick himself. The current paper extends our previous analysis by looking at the value and distribution of the loans which were sanctioned by the General Boards of Health and the Privy Council before 1871 and at their potential impact on the decline of mortality from the 1850 s onwards. It also extends our analysis of the role played by local authority loans after 1870 by adding details of the loans sanctioned by the Local Government Board under other Acts after that date, and the loans which were approved by Parliament under a succession of Local Acts from the 1870s onwards. This new information will provide a much fuller picture of the full range of loans contracted by local authorities during this period and a fuller test of their impact on mortality change.

\section{O-50: Into the past: Nutritional implications of our evo- lutionary history}

S. Márquez, ${ }^{1}$ A. Pagano, ${ }^{2}$ R. Feinman, ${ }^{1}$ M. Markell, ${ }^{3}$ and C. Brown $^{3}$

${ }^{1}$ Departments of Cell Biology \& Otolaryngology, SUNY Downstate Medical Center, New York, USA

${ }^{2}$ Department of Cell Biology, New York University School of Medicine, New York, USA

${ }^{3}$ Department of Nephrology-Division of Renal Disease, SUNY Downstate Medical Center, New York, USA

\section{E-mail: samuel.marquez@downstate.edu}

Keywords: diet; metabolic diseases; dietary evolution

Most primate species are frugivorous (fruit-eating) supplementing their diet with either high calorie foods (insects, tree gums) or with low calorie foods (leaves). Our genus Homo likely evolved from such frugivorous ancestors. Faced with selective pressures of long dry winters, with favored fruits seasonally restricted, early Homo (circa 2.5 million years ago) began producing stone tools to utilize meat resources. They later gave rise to the species Homo erectus, adapted for walking longer distances in search of food. Continued incorporation of meat into the Homo 
erectus diet has been inferred from archeological evidence. As a high calorie, high protein food, meat also led to larger brain size and gut reduction. Despite the emergence of anatomically modern humans around 200,000 years ago, it was not until 100,000 years later that novel foods such as birds, fish, and shellfish were utilized. Only at 10,000 years ago do we see sedentary agriculture appear in the Near East, followed by China and other centers. Early agriculturalists accessed greater amounts of calories but suffered in overall health. Today, an overabundance of simple carbohydrates characterizes the age of industrialized food production and refinement. Overwhelming evidence suggests that this dietary regime is tied to disruption in the response to insulin, resulting in the so-called metabolic syndrome (MetS) characterized by several physiologic disorders including abnormal levels of blood lipid markers (atherogenic dyslipidemia). MetS predicts progression to obesity, diabetes, hypertension, and cardiovascular disease, all of which may respond to dietary carbohydrate restriction. With the perspective of evolutionary medicine, we can see that nutritionally related diseases may be rooted in an evolutionary mismatch between human physiology and the modern diet. This new discipline has seen an explosion of research by physicians and evolutionary biologists asking "why" instead of "how" illness occurs, with the ultimate goal of improving patient care.

\section{O-51: The Egyptian Mummy Project: Paleoepidemio- logic observations}

\section{F. DeWolfe Miller}

John A Burns School of Medicine, University of Hawaii, Honolulu, Hawaii, USA

E-mail: dewoflemiller@gmail.com

Keywords: Egyptian mummies; paleoepidemiology; ancient diseases; conservation

The Egyptian Mummy Project was created with an aim to study health and disease in ancient Egypt. In brief, the method was to first develop a database of all the mummies in Egypt. This was and is an ongoing documentation phase, as no such complete inventory exists. The project obtained a portable Siemens CT scanning machine, currently located at the Egyptian Museum in Cairo. The database currently includes approximately $75 \%$ of all known Egyptian mummies located in Egypt. This is sufficient for basic descriptive paleoepidemiologically comparisons between different types and locations of mummies, which will be shown. Intriguing observations will be given such as Queen Hatshepsut's metastatic cancer, Tut's patella, and the arrowhead in the left chest of Tutmosis I among others. The most overwhelming conclusion from this project to date is the urgent need for mummy conservation for which the database is providing the most striking examples.
O-52: Expanding the understanding of evolution in medical students via a student-run interest group

M. Blyth, J. McNary, E. Lindsey, and M. Hiller

Louisiana State University, USA

E-mail: bucky60@gmail.com

Keywords: team-based learning; journal club; outreach

In order to grow emerging physicians' understanding and application of evolution and medical research, an evolutionary medicine interest group was founded by a first year medical student at Louisiana State University School of Medicine at New Orleans (LSUSOM-NO). Currently in its third year, the group has continued to meet and recruit underclassmen from subsequent entering classes. Participants were approved by the school to receive credit hours towards the school's "professional development" elective. The group follows a journal-club style, with the presenting student choosing journal articles and leading discussion relevant to the month's topic. The organization of topics seeks to augment current medical curricula in microbiology, neuroscience, psychology, genetics, and structural biology while creating an interdisciplinary environment between medicine, research, and public health. Recently, the group also began inviting local experts to give seminars that are open to the entire campus. We believe that studying the principles of evolutionary biology in medicine will serve students on their path to becoming successful physicians and researchers by encouraging a new framework from which to examine health and disease. We hope to share our experiences and methods with the evolutionary medicine community in order to help facilitate and encourage similar groups throughout the country and internationally.

O-53: No place to hide: Evolutionary forensics in virus transmissions and outbreaks

\section{F. González Candelas}

Unidad Mixta Infección y Salud Pública FISABIO-Univ, Valencia

\section{E-mail:fernando.gonzalez@uv.es}

Keywords: molecular phylogenetics; hepatitis C virus; transmission; maximum likelihood

Molecular phylogenetic analyses are used increasingly in the epidemiological investigations of outbreaks and transmission cases involving rapidly evolving RNA viruses. We have developed a procedure to integrate molecular phylogenetic reconstructions into a forensic setting adequate for the analysis of outbreaks and transmission events. The procedure, which necessarily includes nongenetic information, allows for an individual evaluation of the likelihood of each individual in a cohort of suspected victims to have been 
infected from a common source. We have applied this procedure to a large outbreak of hepatitis $\mathrm{C}$ virus caused by a single, continuously evolving source and the results obtained had a key role in the trial that led to the conviction of the suspected source.

We present the results of such an analysis which contributed to the conviction of an anesthetist who likely had infected several hundreds of his patients with HCV. We obtained viral sequences from 322 suspected victims and 44 local, unrelated controls. Our analysis of over 4,000 sequences from the E1-E2 region of the HCV genome allowed us to exclude 47 patients from the outbreak because they did not share a most recent common ancestor with those derived from the suspected source.

Because we knew the dates of infection for some patients, we were able to use these data to validate a Bayesian method with varying molecular clock rates and reconstruct a probability interval for the estimated dates of infection for each patient and the source. The patients were all infected between 1988 and 1998, shortly after the estimated date of infection of the source. These dates were largely in agreement with those derived from documental evidence by the prosecution.

These results were used in court and helped to convict the presumed source for medical malpractice that led to a sentence of almost 2,000 years in prison.

\section{O-54: Diversifying our evidence: Evolutionary medicine} and the potentials and pitfalls of text-based sources

$\underline{\text { K. van Schaik }}$

Harvard Medical School and Harvard Department of the Classics, Harvard University, USA

E-mail: Katherine_vanSchaik@hms.harvard.edu

Keywords: paleopathology; text; Galen; hippocrates

Evolutionary medicine seeks to apply evolutionary theory to concepts of health and disease, with the goal of understanding not only how we get sick, but also why we get sick. Research in genetics and in comparative anatomy is foundational for the field, and advances in knowledge are made as we use data from such studies to trace the evolution of life through the past and into the present. Scientific analysis can teach us much about this past, but what about other sources of information about the health and disease of past populations? This paper will argue that an examination of medical texts from ancient Babylon, Egypt, Greece, and Rome (spanning a period from the second millennium $\mathrm{BCE}$ to the third century $\mathrm{CE}$ ) will provide vital insight into burden of disease and human responses to disease that cannot be derived from scientific analysis alone. While texts are limited in the benefits they can provide to the field of evolutionary medicine, an interdisciplinary approach, favoring combined investigation of past burden of disease through material evidence and through textual, provides a nuanced understanding of human responses to epidemics, chronic disease, child mortality, and other realities of human existence.

\section{O-55: Secular trends in weight and height and their} interrelationship in Swiss conscripts over 130 years

$\underline{\text { K. Staub }},{ }^{1}$ N. Bender, ${ }^{2}$ and F. Rühli ${ }^{1}$

${ }^{1}$ Institute of Evolutionary Medicine, University of Zurich, Switzerland

${ }^{2}$ Institute of Social and Preventive Medicine, University of Bern, Switzerland

E-mail: kaspar.staub@iem.uzh.ch

Keywords: obesity; stature; 19th century; mismatch

Background. The recent obesity pandemic represents a response to changing environments and has been addressed by different hypotheses, like the thrifty gene hypothesis, thrifty phenotype hypothesis, mismatch hypothesis, life course hypothesis, and so on. Here we add individual Body Mass Index data from Swiss conscription 1875-2014 to this discussion. Data. The Swiss standardized and universal recruitment procedure remained unchanged since 1875 and thus offers a valuable data source for temporal analyses. The population of conscripts was, and still is, almost identical (90\%-100\%) with the Swiss resident male population aged $18 \mathrm{y}-20 \mathrm{y}$. Body measurements have been taken by medics for all conscripts (whether capable of serving or not). We included individual data (age 18y-20y) for 1875-1879 ( $N=7$ '514, all existing weight data), for 1933-1939 $(N=15,450)$, and for 1992-2014 $(N=679,938)$ in our analysis. Results. In $2014(N=32,973)$, average height was $178.35 \mathrm{~cm}$ (95\% CI 178.28-178.42) and average weight $74.16 \mathrm{~kg}$ (74.01-74.30). Average BMI was $23.29 \mathrm{~kg} / \mathrm{m}^{2}$ (23.25-23.33). The weight and BMI distributions were clearly right skewed (skewness 1.38 and 1.58). The prevalence of overweight (BMI $25.0-29.9 \mathrm{~kg} / \mathrm{m}^{2}$ ) was $18.4 \%(17.9-18.9)$ and obesity (BMI $\left.\geq 30.0 \mathrm{~kg} / \mathrm{m}^{2}\right) 5.7 \%$ (5.3-6.1). The long-term trend shows that unlike height, weight did not stabilize in recent decades: average BMI shows a two-step increase at the beginning of the 1990s and since 2002. However, since 2009/2010 the increase in weight and BMI has come to a halt, the obesity epidemic seems to plateau. Back in 1875-1879, height was leftskewed (-0.35), short men were overrepresented. BMI was nearly normally distributed, but $12.1 \%$ of the young men were underweight (overweight and obesity were relatively rare). Since 1994, BMI became increasingly right-skewed, in 2014 a total of $24.1 \%$ of the young Swiss men were overweight or obese $\left(\mathrm{BMI} \geq 25.0 \mathrm{~kg} / \mathrm{m}^{2}\right)$. Also, the BMI per height relation changed: in 1875-1879 the relation was positive between the 5th and the 95th height centile 
(being taller $=$ higher BMI). Very short men (below the 5th height centile) were particularly thin. In contrast, in the 1930s, 1994, and 2014 the BMI per height relation is negative (being taller = lower BMI). Discussion. The changes in height and BMI among Swiss conscripts reflect the development from a 19th century society (body-)shaped by undernutrition to a modern overnourished society. Our results indicate that there is evidence for the contrastingly different relation between an unstable food environment and the shape of the human body even at the eve of the industrialization in the second half of the 19th century in Switzerland (confirmation of the mismatch hypothesis and/or the life course hypothesis). Since growth in height has plateaued in the stable environment of the modern society (due to a reached genetic maximum) the human body seems to have invested the surplus energy rather in breadth growth.

O-56: Evolutionary medicine as part of the medical curriculum in Switzerland and Australia: a qualitative and quantitative feedback

$\underline{\text { F. Rühli }},{ }^{1}$ N. Bender,${ }^{2}$ A. Saniotis, ${ }^{3}$ and M. Henneberg ${ }^{3}$

${ }^{1}$ Institute of Evolutionary Medicine, Medical Faculty, University of Zurich, Switzerland

${ }^{2}$ Institute of Social and Preventive Medicine, Medical Faculty, University of Bern, Switzerland

${ }^{3}$ Biological Anthropology and Comparative Anatomy Unit, School of Medical Sciences, The University of Adelaide, Australia

E-mail:frank.ruehli@iem.uzh.ch

Keywords: elective; medical school; microevolution; teaching

Evolutionary medicine is a growing transdisciplinary research and teaching field. The incorporation into the medical school introduces principles of human evolution and its forces into the knowledge of future practitioners of health-related professions. Based on our unique experience, which now spans several years at three locations (Zurich, Bern, Adelaide), we have gained helpful feedback by those involved in teaching as well as by the students on how to best teach evolutionary medicine as part of an elective or as mandatory lectures in the medical education. The aim of this presentation is not only to address the general challenges (teaching and exam format, most suitable topics for lectures and students presentations, etc.) of such teaching but also to discuss the qualitative and quantitative feedback students gave. We think that our experience is most valuable since teaching evolutionary medicine as part of the medical curricula is different than for the science curriculum and it will be crucial for the long-term impact of this novel transdisciplinary field within the medical community.
0-57: What you can tell from a smile: How ancient biomolecules recovered from the Medieval Dalheim cemetery inform our past

A. Bouwman, ${ }^{1}$ C. Warinner, ${ }^{1,2}$ A. Krüttli, ${ }^{1}$ and F. Rühli ${ }^{1}$

${ }^{1}$ Institute for Evolutionary Medicine, University of Zurich, Switzerland

${ }^{2}$ Laboratories of Molecular Anthropology and Microbiome Research, University of Oklahoma, USA

E-mail: abigail.bouwman@uzh.ch

Keywords: aDNA; proteomics; archeology

The medieval cemetery of Dalheim, Germany, was host to a religious community and lay people alike. The site was excavated from 1989 and the remains carefully studied osteologically before being given to the University of Zurich. Since 2010, the remains have been studied using biomolecular techniques including conventional targeted PCR amplification and sequencing, high-throughput metagenomics sequencing and tandem mass spectrometrybased metaproteomics, to test various hypothesis about the health and diets of medieval Europeans within a wider evolutionary medicine context.

Here we will present a summary of the data that we obtained which underlines the importance of medium to large-scale skeletal collections in furthering our understanding of the evolution of human health. For example, the lactase-persistence -13910T allele frequency within the site was similar to the current German allele frequency showing that the allele had risen to today's frequency by the Medieval period, implying that unprocessed milk consumption contributed to evolutionary adaptation over a relatively short period of time.

Combining archeological and ancient molecular data from this site and others around Europe we can obtain a much clearer view of our past.

\section{O-58: Are our back problems evolutionary trade-offs of bipedalism?}

M. Haeusler, N. Frater, R. Hangartner, C. Minder, T. Böni, and F. Rühli

Institute of Evolutionary Medicine, University of Zurich, Switzerland

E-mail: martin.haeusler@iem.uzh.ch

Keywords: paleopathology; low back pain

Back problems are the foremost musculoskeletal disorder of modern humans that affect nearly all of us at some point in life. Often, they are explained by the sedentary lifestyle of Western populations, muscular disuse, or some other mismatch between environment and design. However, there is no compelling evidence that the prevalence of back problems has changed in the last decades nor that it is significantly different throughout the world. Moreover, 
a series of twin studies demonstrated that genetic factors are several times more important to explain back problems than environmental factors including inadequate physical loading. Nevertheless, the majority of the variability of spinal degenerative disease remained unexplained in these studies. Part of this might be caused by our everyday exposure to bipedal locomotion and the specific mechanical strains it exerts on our spine. In fact, spinal pathologies are remarkably uncommon in quadrupedal great apes. Here, we test the hypothesis that our back problems can be attributed to the evolutionary trade-offs of bipedalism. In an analysis of the hominid fossil record we found a remarkably high prevalence of vertebral pathologies including Scheuermann's disease, juvenile disc herniation and spondylolisthesis that all are related to biomechanical stress during adolescence. Crucially, vertebral cross-sectional area relative to body size was found to be markedly smaller in early hominids compared to modern humans, which likely contributed to biomechanical stress. Furthermore, it has been proposed that the adoption of bipedalism in early hominids was facilitated by a longer, more mobile lumbar spine. This too was probably biomechanically disadvantageous as suggested by a preliminary analysis of normal variants in radiographs of modern low back patients. Consequently, the modern human spinal column seems to have been shaped by natural selection to become less vulnerable than that of our ancestors, resulting in an optimized compromise between mobility and stability.

P-01: Reconceptualizing health in Japan: How individual responsibility and empowerment turned into key factors for a prosperous nation

\section{T. Huellein}

Asia-Orient-Institute, URPP Asia and Europe, University of Zurich, Switzerland

\section{E-mail: thomas.huellein@uzh.ch}

Keywords: public health; lifestyle related diseases; normalization

"Lifestyle-related diseases" (e.g., diabetes, cancer, and cardiovascular diseases) are difficult to treat if not incurable. They are the most common causes of death in Japan and other industrialized and some developing countries. They require long-term therapy, usually involving expensive medication and/or technology. Empirical evidence indicates causality between individual behavior and "lifestyle-related diseases," spurring Japanese health policy to educate the population on healthy lifestyles. "Producing Health" is the new buzzword, establishing a public health perspective on most everyday activities.

This has an impact on the concept of health itself, because individual behavior through the entire lifespan becomes relevant to (future) states of health. Even without any symptoms, every individual has specific risk factors, resulting from genetic disposition, behavior, and environmental factors. These will inevitably lead to diseases at some point in the future and therefore, from the perspective of "producing health," need to be "managed," "controlled" and "reduced" as much as possible by the individual. Experts must produce "true" knowledge about risk factors and health while recipients of this knowledge (nonexperts) are supposed to alter their behavior accordingly. How does the meaning of "health" change when the entire life span becomes relevant to health promotion? Who creates "true" knowledge concerning "healthy" lifestyles and how is this knowledge related to power? How is individual health connected to national prosperity under these circumstances? Where is the line between "health" and "disease" if referential frameworks are based on risk factors measured in mathematical probabilities instead of precisely defined symptoms? The talk will address these questions and provide information derived from an ongoing research project funded by the Swiss National Science Foundation. "Health" is analyzed as a global phenomenon that is historically and socially constructed and undergoing alterations.

\section{P-02: HPV positive breast cancers in the light of evolu- tion}

\section{$\underline{\text { P. Vinodbhai }}$}

\section{Indian Astrobiology Research Centre, India}

E-mail: nitesh.iarc@gmail.com

Keywords: human papillomavirus; breast cancer; evolution and tissue tropism

Persistent infection of high-risk HPV types is considered to be the major risk factor for anogenital cancers all over the world. Numerous studies since 1992 have detected HPV DNA in tissues of breast cancer all over the world. Majority of authors have claimed that high risk HPV type might be playing etiological role in breast cancer too. Interpretation of current data on the role of HPV in breast tumors from an evolutionary perspective can offer useful insights. The evidence for transcriptional activity and mRNA expression of genes responsible for malignant transformation like E6 and E7 is completely lacking in breast cancer cases. This dilutes the credibility of the claims on the role of HPV in breast cancer tissues. It is also not clear on how some of the detected low risk HPV types are able to cause breast cancer. The life cycle of HPVs is tightly linked to the differentiation program of human keratinocytes of cutaneous and mucosal region. Breast tissue on the other hand has very limited amount of keratinocytes, restricted to glandular ones in mammary duct and cutaneous type in nipple. Squamous cell carcinoma is signature malignancy of HPV but it is very rare cancer type in breast tissue. On the other hand, the HPV 
types that are strictly associated with mucosal lesions have been detected in cutaneous keratinocytes of breast nipple. This raises question on the prevalent dogma of HPV tissue tropism of cutaneous and mucosal type. Persistent colonization in breast mammary ducts will be an evolutionary dead end for the virus if it is not shed in large amount via milk. The same might not be the case for HPV in oral cavity as it can shed and increase its interhost transmission through deep mouth kissing.

\section{P-03: Where to next? When should we consider rescan- ning mummies?}

R. Loynes

KNH Centre for Biomedical Egyptology, University of Manchester, UK

E-mail: robert.loynes@manchester.ac.uk

Keywords: Egypt; mummy; CT scan; future

With the continuing and inexorable march of technical advances in medical imaging, the subject of re-examination needs to be considered. At what point should CT scan image files of ancient artefacts be deemed out of date and in need of refreshment using current, modern equipment?

A consideration of the history of medical imaging indicates that constant review of available imaging techniques and the quality of acquired data files should be undertaken.

The inherent risks of transport, etcetera must be weighed against the advantages of new, improved data. However, with modern conservation techniques, re-examination should be seriously considered when thought appropriate.

This poster will consider current CT technology and Dicom Reader software. It will also discuss current trends. Problems arising from historic data files and old software will also be considered alongside the problems occurring as the result of radiographic parameters being suboptimal.

\section{P-04: Functional Genomics Center Zurich: The core lab of the Zurich Life Science community}

\section{Poveda, P. Nanni, N. Selevsek, and R. Schlapbach}

Functional Genomics Center Zurich, University Zurich and ETH Zurich, Switzerland

E-mail: lucy.poveda@fgcz.uzh.ch

Keywords: functional genomics core lab

The Functional Genomics Center Zurich (FGCZ) is a joint state-of-the-art research and training facility of the ETH Zurich and the University of Zurich. With the latest technologies and key expertise for Omics research, the FGCZ carries out research projects and technology development in collaboration with the Zurich Life Science research community.

New analytical approaches in the areas of genomics, transcriptomics, proteomics, and metabolomics are being developed to answer the variety of biological questions. Using ultrahigh throughput sequencing systems and mass spectrometry-based technologies, molecular measurements at DNA, RNA, protein, and metabolite levels can be investigated for the elucidation of biological and cellular systems.

P-05: Hitch-hiking to the South West Pacific: understanding the mitochondrial genetic diversity of Plasmodium vivax malaria in Melanesia through its connection with past human migrations

L. Raijmakers, ${ }^{1,2}$ A. Harrison, ${ }^{2}$ I. Felger, ${ }^{3}$ C. Koepfli, ${ }^{2}$ A. Bogaard, ${ }^{4}$ P. Rodrigues, ${ }^{5}$ A. Barry, ${ }^{2}$ and I. Mueller ${ }^{2}$

${ }^{1}$ Research Lab for Archaeology and the History of Art, University of Oxford, $U K$

${ }^{2}$ Population Health and Immunity Division, The Walter and Eliza Hall Institute of Medical Research, Parkville, Victoria, Australia

${ }^{3}$ Medical Parasitology and Infection Biology, Swiss TPH, Basle, Switzerland

${ }^{4}$ Institute of Archaeology, University of Oxford, UK

${ }^{5}$ Brazil Institute of Biomedical Sciences, University of Sao Paulo, Brasil

E-mail: leonie.raijmakers@merton.ox.ac.uk

Keywords: malaria; migration; disease; evolution

Plasmodium vivax malaria is geographically the most widespread of human malaria species. Globally, P. vivax exhibits considerable genetic diversity, the origins and drivers of which are still largely unknown. It is likely that some of this diversity can be attributed to the long evolutionary history of $P$. vivax and potentially complex association of $P$. vivax distributions with past human migratory movements. To this effect, we have focused on understanding the origin(s) and age of the highly variable $P$. vivax in and around Melanesia through mapping the diversity in mitochondrial genomes from over 200 P. vivax samples from Papua New Guinea, Solomon Islands, and Vanuatu, and relating it to the major past human migration events in the SW Pacific, namely: Papuan (at 60.000-20.000 years ago) and Austronesian (at 6.000-1.000 years ago).

The interest in understanding the mutual past of $P$. vivax and humans in the Melanesian region is due to the pronounced genetic diversity found in both, which includes numerous genetic adaptations to malaria by all human populations present before European settlement. Several of these adaptations suggest a long, continued history of exposure, a finding that could aid in the comprehension of past human population dynamics in the region including patterns of settlement and population sizes. Current results show that most SW Pacific P. vivax populations carry two closely related mitochondrial genome haplotypes (genetic strains). These haplotypes are significantly distinct from the main haplotypes circulating in neighboring East and South East 
Asia, and are more closely related to those present in Africa and South Asia, indicating an early introduction and consecutive isolation of Melanesian P. vivax. We will discuss the implication of these observations further in the context of our knowledge of human colonization of the SW Pacific.

P-06: Bloodletting's success story: Perspectives from evolutionary psychology and cultural transmission

\author{
$\underline{\text { H. Miton, }}{ }^{1}$ N. Claidière, ${ }^{2}$ and H. Mercier ${ }^{3}$ \\ ${ }^{1}$ Université Lyon 2/CNRS, France \\ ${ }^{2}$ Laboratoire de Psychologie Cognitive-CNRS, Université \\ d'Aix-Marseille, France \\ ${ }^{3}$ University of Neuchatel, Switzerland \\ E-mail: helena.miton@gmail.com
}

Keywords: bloodletting; cultural attraction; transmission chains; medical anthropology

Bloodletting - the practice of letting blood out to cure a patient-was for centuries one of the main therapies in the west. We lay out three potential explanations for bloodletting's cultural success: that it was efficient, that it was defended by prestigious sources-in particular ancient physicians-, and that cognitive mechanisms made it a particularly attractive practice. To test these explanations, we first review the anthropological data available in eHRAF. These data reveal that bloodletting is practiced by many unrelated cultures worldwide, where it is performed for different indications and in different ways. This suggests that the success of bloodletting cannot only be explained by its medical efficiency or by the prestige of western physicians. Instead, some universal cognitive mechanisms likely make bloodletting an attractive form of therapy. We further test this hypothesis using the technique of transmission chains. Three experiments are conducted in the U.S., a culture that does not practice bloodletting. Studies 1 and 2 reveal that stories involving bloodletting survive longer than some other common therapies, and that the most successful variants in the experiments are also the most successful variants worldwide. Study 3 shows how a story about a mundane event - an accidental cut—can turn into a story about bloodletting. This research demonstrates the potential of combining different methodologies-review of anthropological data, experiments, and modeling - to investigate cultural phenomena. Also, this research illustrates how evolutionary frameworks on cultural representations can explain the success of maladaptive medical practices.

\section{P-07: Leukemia in the Neolithic-Evidence in cancellous bone of a 7,000 year old skeleton}

\section{H. Scherf, ${ }^{1}$ M. Francken, ${ }^{1}$ J. Wahl, ${ }^{2}$ and K. Harvati ${ }^{1}$}

${ }^{1}$ Paleanthropology, Senckenberg Center for Human Evolution and Paleoenvironment, Eberhard Karls University, Tübingen, Germany
${ }^{2}$ Regierungspräsidium Stuttgart, Landesamt für Denkmalpflege Konstanz, Germany

E-mail: heike.scherf@ifu.uni-tuebingen.de

Keywords: cancellous bone; high resolution CT; structural changes; resorption

The harsh living conditions in Neolithic agricultural settlements can be inferred from various paleopathological signs on skeletal remains. The normal spectrum of pathologies identified is caused by chronic illness, infectious diseases, malnutrition, and trauma. Sometimes evidence for soft tissue tumor activity can be found by pathophysiological remodeling of the cortical bone.

Here we present an interesting case of a female individual of biological age determined as $30-40 \mathrm{y}$, from the early Neolithic Linear Pottery Culture (LBK) site StuttgartMühlhausen in SW Germany. Previously only a severe case of dental caries with alveolar inflammation was reported for this individual. Our high resolution CT study (GE v|tome|x s, University of Tübingen Paleoanthropology High Resolution Computed Tomography Laboratory) revealed a pattern of profound loss of trabecular bone in both humeral and sternal cancellous bone.

The level of trabecular resorption is significantly higher compared to specimens of the same age group from the same site and to recent human samples of the adult age class. The pattern of resorption is indicative for endocrine disorders and neoplastic conditions. Former studies identified at the Neolithic graveyard of Stuttgart-Mühlhausen a case of primary hyperparathyroidism, an endocrine dysfunction causing disintegration of cancellous bone. However, the condition of the present case corresponds more closely with neoplastic conditions. The locally restricted bone resorption in the cancellous bone of sternum and proximal humerus at the sites of hematopoietic stem cell generation strongly suggests a case of early leukemia. Provided that our interpretation is valid, this case represents the oldest known evidence for leukemia.

P-08: Cranial suture closure in domestic dog breeds and relations to skull anatomy-a comparison with human patterns

\section{Geiger, S. Haussman, and M. Sánchez-Villagra}

Paläontologisches Institut und Museum, Universität Zürich, Schweiz

E-mail: madeleine.geiger@pim.uzh.ch

Keywords: wolf; synchondroses; brachycephaly; airorhynchy

Some domestic dog breeds are characterized by a relatively short and broad skull (brachycephaly) and/or a dorsally rotated rostrum (airorhynchy). These skull conformations, together with accompanying peculiarities of the braincase, 
face, and teeth, resemble human syndromes associated with premature fusion of certain craniofacial sutures and cranial base synchondroses, for example, achondroplasia, Apert syndrome, and Crouzon syndrome. In domestic dogs, the association of brachycephaly and airorhynchy with patterns of suture and synchondroses closure is not well investigated. We aim to explore breed-specific patterns of cranial suture closure in relation to degrees of brachycephaly and airorhynchy in domestic dogs. For this purpose, we coded closure of 19 sutures and synchondroses in 46 wolves - the ancestor of all domestic dogs-and 106 domestic dogs comprising 6 breeds. Comparisons of relative closure scores in adult individuals showed that those breeds with the greatest degrees of airorhynchy have significantly more closing and closed sutures than the wolf. Especially the fronto-parietal and the premaxillomaxillary suture tend to be more often closed in the brachycephalic and airorhynch breeds. These results are partially consistent with studies on humans, mice, and rabbits. Closure patterns of other craniofacial sutures and the basispheno-basioccipital synchondrosis in the domestic dog, however, do not mirror so well the mechanisms found in the other species. This suggests that although there are parallels, different mechanisms might act in the generation of the brachycephalic and airorhynch phenotype in domestic dogs and other species. This study underscores the relevance of phenomic comparisons of domesticated mammals with humans to obtain insights into evolutionary patterns and eventually mechanisms of medical significance.

\section{P-09: Birth weight inequality in Basle during World War I}

J. Floris, ${ }^{1}$ K. Staub, ${ }^{2}$ and U. Woitek ${ }^{1}$

${ }^{1}$ Department of Economics, University of Zurich, Switzerland

${ }^{2}$ Institute of Evolutionary Medicine, University of Zurich, Switzerland

E-mail: joel.floris@econ.uzh.ch

Keywords: anthropometrics; birth weight; social inequality; standard of living

It is well known that in past and present populations, fetal development is strongly affected by maternal nutritional status during pregnancy. Furthermore, there is an interrelationship between infant size, economic development, and socioeconomic background of mothers. Therefore, birth weight is a useful measure to analyze women's living conditions in the past. At the same time, there is an increasing volume of literature on long-running effects of early life conditions on mortality or health in later life.

In this study, we analyze individual anthropometric and socioeconomic information from case records kept at the University Maternity Hospital in the City of Basle, where information about each maternity patient and newborn child have been routinely recorded since the 19th century. We focus on World War I to explore socioeconomic differences in size of new-borns and consequently in maternal living conditions in a time of emerging crisis with particularly harsh times of malnutrition in 1918 and 1919.

Relying on birth records from the hospital has two advantages: firstly, around $60 \%$ of all children in one year in the city were born there. Thus, the data covers a comparatively large share of births, including women from all levels of the socioeconomic strata. Secondly, the birth records contain an extensive amount of detailed information allowing us to control for other relevant biological determinants of infant size. We used a mother's residence address and occupation as well as the occupation of the fathers to indicate the socioeconomic background. In addition, we can rely on social and economic data collected by the statistical office of the city, as well as on several published household expenditure data to complement the information on economic and social conditions around birth.

Although Switzerland did not participate in the war, trade relations with the surrounding belligerent countries were severed. This affected both exports and, more importantly for our research question, food imports: in the period 1906-1922, 84\% of the domestical consumed grains and grain products had to be imported. As the men were called to arms, work intensity for women increased. The government started to intervene as early as 1914 to mitigate the situation, introducing price limits and food rationing.

Our preliminary results show a negative effect on women's living conditions reflected in birth weight. While the height of the mother had on average a positive effect on birth weight (about $9 \mathrm{~g} / \mathrm{cm}$ ), the fact that the mother had to work at a factory reduced birth weight by about $124 \mathrm{~g}$. The influence of undernutrition of the mother, multiple births, parity, still-birth, sex, height and age of the mother, and gestational age also have a significant impact. A low socioeconomic position of both parents leads to a lower birth weight. We find a significant decrease in average birth weight 1,918 and 1,919. Interestingly, the decrease is the same across all socioeconomic positions, indicating that interventions such as food rationing at least prevented an increase in inequality.

P-10: Secular change of femoral morphology from a clinical perspective: Would a modern anatomically precontoured fracture fixation implant fit medieval femora?

B. Schmutz, ${ }^{1}$ F. Schuetz, ${ }^{1}$ M. Schuetz, ${ }^{1,2}$ and F. Rühli ${ }^{3}$

${ }^{1}$ Institute of Health \& Biomedical Innovation, Queensland University of Technology, Brisbane, Australia

${ }^{2}$ Trauma Service, Princess Alexandra Hospital, Brisbane, Australia 
${ }^{3}$ Institute of Evolutionary Medicine, University of Zurich, Zurich, Switzerland

E-mail:b.schmutz@qut.edu.au

Keywords: secular change; femur; intramedullary nail; anatomical fit

Current treatment for internal fixation of bone fractures requires metal implants (plates and intramedullary nails) which are optimally contoured to fit the specific shape of patient's bones. As bone morphology is influenced by factors such as nutrition and activity level, there is a high clinical interest on acquiring data for the design of such implants from specific populations. However, little is known of how secular changes impact the implant design.

Therefore, using femora from the Dalheim Collection (NW Germany, dated ca. 1300AD) as example, this study aims to investigate and quantify to what extent femoral morphology might have changed over the last 700 years in relation to implant design.

Virtual 3D models reconstructed from computed tomography data of 20 femora from the Dalheim Collection and from 31 modern Caucasian (Switzerland, North America) are utilized. With the aid of a custom designed software tool, 3D models of a recent proximal femoral nail design were virtually implanted into $3 \mathrm{D}$ models representing the inner cortical surface of the intact bones. Anatomical nail fit was determined from the total surface area of nail protrusion from the inner cortex surface and maximum distance of nail protrusion in the axial plane. To assess the impact of femoral antecurvature on nail fit, the position of the distal nail tip within the medullary cavity was quantified.

Initial results show a considerably larger anatomical misfit (mean: $1,232 \mathrm{~mm}^{2}, 2.5 \mathrm{~mm}$ ) of the nail for the medieval $(n=7)$ bones compared to the modern $(n=31)$ bones (mean: $583 \mathrm{~mm}^{2}, 1.3 \mathrm{~mm}$ ). In all but one of the analyzed medieval bones, the nail tip was located anterior in the canal versus a distribution $(55 \%$ anterior, $35 \%$ center, $10 \%$ posterior) in the modern bones.

This suggests that secular change over the last 700 years might have had a considerable impact on femoral morphology in relation to implant design.

\section{P-11: Which technique is best? Optimizing MRI of an ancient mummified human hand}

A. Özen, ${ }^{1}$ U. Ludwig, ${ }^{1}$ L. Öhrström, ${ }^{2}$ F. Rühli, ${ }^{2}$ and M. Bock $^{1}$

${ }^{1}$ Radiology - Medical Physics, University Medical Center Freiburg, Freiburg, Germany

${ }^{2}$ Institute of Evolutionary Medicine, University of Zurich, Zurich, Switzerland

E-mail: ali.oezen@uniklinik-freiburg.de

Keywords: magnetic resonance imaging; ancient human mummy; short echo time; relaxation time
MRI of mummified tissue is challenging due to the extremely low water content and the very short transverse relaxation times $\mathrm{T} 2^{*}$. To overcome the signal loss associated with the short T2*, 3 pulse sequences with very short echo times (TE) were compared for MRI of ancient remains in terms of signal-to-noise ratio efficiency: MR ultrashort echo time (UTE), pointwise encoding time reduction with radial acquisition (PETRA), and single point imaging (SPI). Images of an ancient mummified human hand were acquired at field strengths of $1.5 \mathrm{~T}$ and $3 \mathrm{~T}$ using homemade solenoid radio frequency coils. At $3 \mathrm{~T}$, the impact of a high-performance gradient system $(80 \mathrm{mT} / \mathrm{m})$ on the image quality was assessed.

In all MR images, mummified tissues could be differentiated and anatomical structures such as bones and tendons were clearly identified. Skin with embalming resin was hyperintense in MRI, whereas it appeared isointense in comparative CT data. PETRA showed the highest signal-to-noise ratio. With UTE, short scan times and a homogeneous RF excitation could be achieved, and blurring was less pronounced than with PETRA. SPI showed no blurring artifacts; however, it required very long scan times. Measurements of the relaxation times showed that $\mathrm{T} 1$ of this sample was only slightly smaller than typical in vivo T1 values at the same field strength. T2* values, however, are more than one order of magnitude smaller than in humans, which necessitates the use of ultrashort imaging techniques. To achieve very short TEs, the use of high fidelity gradient systems proved to be beneficial, as well as the use of Tx/Rx coils.

This work provided an initial analysis for the optimization of imaging protocols for paleoradiology studies with MRI, and, ultimately, for MRI of tissue with extremely short $\mathrm{T} 2 *$.

P-12: Insulin-amylin and $\beta$-cell dynamics model to differentiate between alternative evolutionary hypotheses for type 2 diabetes mellitus

$\underline{\text { M. Diwekar-Joshi, A. Bodas, and M. Watve }}$

Department of Biological Sciences, Indian Institute of Science Education and Research, Pune, India

E-mail: milind@iiserpune.ac.in

Keywords: T2DM; pancreatic $\beta$-cells; thrift; neurobehavioral origins hypothesis

The thrifty gene or thrifty phenotype hypotheses have dominated evolutionary thinking in type 2 diabetes mellitus (T2DM). This is recently challenged by the neorobehavioral origins hypothesis working mainly through neuronal mechanisms. We use the $\beta$-cell related predictions of the two hypotheses to differentially test them. In type 1 diabetes mellitus (T1DM), there is almost total destruction of $\beta$-cells, whereas a substantial mass survives life-long in T2DM. 
Amyloid deposits are significantly associated with $\beta$-cell degeneration in T2DM but not in T1DM. We construct a mathematical model of the intracellular dynamics of insulin and islet amyloid pancreatic polypeptide (IAPP). A parameter independent result of the model is that contrary to popular belief, insulin resistance, compensatory insulin response, hyperglycemia, and glucotoxicity always arrest amyloidogenesis rather than increase it. On the other hand, sympathetic suppression of insulin release can facilitate amyloidogenesis. A combination of sympathetic suppression and amyloid toxicity works in a negative feedback cycle and allows indefinite survival of a subnormal $\beta$-cell population. The classical model, in contrast, leads to a positive feedback-mediated accelerated degeneration of $\beta$-cell mass ending in complete loss of the population which is not a characteristic of T2DM. Thus the predictions of the neurobehavioral origins model rather than the classical evolutionary model are better supported by the $\beta$-cell dynamics.

\section{P-13: Distinct statistical signatures of peripheral and central mechanisms of glucose regulation}

\section{Diwekar-Joshi, A. Chatterjee, P. Patil, and M. Watve}

Department of Biological Sciences, Indian Institute of Science Education and Research, Pune, India

E-mail:milind@iiserpune.ac.in

Keywords: OGTT; glucose homeostasis; central nervous system; glut-1

By the current mainstream thinking, a combination of insulin resistance and $\beta$-cell dysfunction causes hyperglycemia in type 2 diabetes mellitus (T2DM). However, there is also evidence for the role of the central nervous system in glucose homeostasis. The peripheral or insulincentered model follows the classical theory of thrift and the central model follows the neurobehavioral origins theory of T2DM. In order to resolve between alternative hypotheses, we use mathematical models to make multiple testable predictions from each of the putative regulatory mechanisms and test them against oral glucose tolerance test (OGTT) data. The three predictions are about (i) independent effects of fasting glucose and the area under the curve in OGTT, (ii) a correlation between glucose and insulin during the OGTT curve, and (iii) a delay in insulin response in diabetics as compared to healthy subjects. On comparison of predictions with OGTT data of normal subjects as well as diabetics, it is seen that the insulin-centered peripheral models of glucose homeostasis are unable to explain many of the parameters of an altered OGTT. The CNS-based regulatory pathway for glucose homeostasis explains these changes better. The CNS-based model states that a central shift in regulatory mechanisms and downregulation of the glucose transporter glut-1 in the blood brain barrier are the main reasons for diabetic hyperglycemia. A combination of insulin resistance and $\beta$-cell dysfunction is unlikely to be necessary and sufficient cause of hyperglycemia and the role of CNS is highlighted. This warrants a radical rethinking of the causes of hyperglycemia in T2DM as well as that of the evolutionary origins of the disorder.

\section{P-14: What Lotka-Volterra competition model teaches us about type 2 diabetes mellitus}

M. Diwekar-Joshi, S. Chawla, A. Pund, S. Kulkarni, and M. Watve

Department of Biological Sciences, Indian Institute of Science Education and Research, Pune, India

E-mail: milind@iiserpune.ac.in

Keywords: biological causation; Lotka-Volterra model; glucose homeostasis

The nature of causation in biology and reliable methods for drawing causal inferences is still very hazy. It is generally agreed that interventional but not correlational data indicate causation. We argue here that the nature of causal relations can be different in perturbed states versus steady states. For example in the Lotka-Volterra (LV) model of competition the growth rates $(r)$ of competing species are not the determinants of their equilibrium populations. Alterations in $r$ do not shift the LV equilibrium but affect the rate at which the system returns to equilibrium after a perturbation. Most physiological experiments are perturbation experiments and therefore not sufficient to establish steady state causation in a homeostatic system. We discuss the specific case of plasma glucose regulation by insulin and show that although plasma glucose stimulates insulin production and insulin in turn regulates plasma glucose levels, there is no evidence that insulin determines glucose levels in a fasting steady state. The weak correlation between insulin and glucose in steady state, difference in regression and correlation parameters in steady versus post meal state, and experiments on sustained alterations in glucose or insulin levels together indicate that the demonstrated relationship between glucose and insulin in perturbation experiments does not hold true in the fasting steady state. The behavior of insulin in glucose homeostasis is similar to the behavior of $r$ in LV. Insulin helps bringing back glucose levels to a steady state but does not appear to decide the steady state levels of glucose. Applying principles learned from ecology to pathophysiology can resolve many long-standing paradoxes in diabetes.

\section{P-15: Is whole genome sequencing (WGS) the better whole exome sequencing (WES)?}

J. Meienberg, ${ }^{1}$ K. Zerjavic, ${ }^{1}$ I. Keller, ${ }^{2}$ M. Okoniewski, ${ }^{3,4}$ A. Patrignani, ${ }^{3}$ K. Ludin, ${ }^{5}$ Z. Xu, ${ }^{6}$ B. Steinmann, ${ }^{7}$ T. Carrel, ${ }^{8}$ B. Röthlisberger, ${ }^{5}$ R. Schlapbach, ${ }^{3}$ R. Bruggmann, ${ }^{9}$ and G. Matyas ${ }^{1,8,10}$ 
${ }^{1}$ Center for Cardiovascular Genetics and Gene Diagnostics, Foundation for People with Rare Diseases, SchlierenZurich, Switzerland

${ }^{2}$ Department of Clinical Research, University of Berne, Berne, Switzerland

${ }^{3}$ Functional Genomics Center Zurich, Zurich, Switzerland

${ }^{4}$ Division of Scientific IT Services, ETH Zurich, Zurich, Switzerland

${ }^{5}$ Division of Medical Genetics, Center for Laboratory Medicine, Aarau, Switzerland

${ }^{6}$ Sophia Genetics SA, Saint Sulpice, Switzerland

${ }^{7}$ Division of Metabolism, University Children's Hospital, Zurich, Switzerland

${ }^{8}$ Department of Cardiovascular Surgery, University Hospital, Berne, Switzerland

${ }^{9}$ Interfaculty Bioinformatics Unit and Swiss Institute of Bioinformatics, University of Berne, Berne, Switzerland

${ }^{10}$ Zurich Center for Integrative Human Physiology, University of Zurich, Zurich, Switzerland

E-mail: meienberg@genetikzentrum.ch

Keywords: exome coverage; next generation sequencing; whole exome sequencing; whole genome sequencing

Whole exome sequencing (WES) is increasingly used in research and diagnostics. WES users expect coverage of the entire coding region of known genes as well as sufficient read depth for the covered regions. It is, however, unknown which recent WES platform is most suitable to meet these expectations. We present insights into the performance of the most recent standard exome enrichment platforms from Agilent, NimbleGen, and Illumina applied to six different DNA samples by two sequencing vendors per platform as well as whole genome sequencing (WGS) performed on different sequencing platforms including the most recent HiSeq X Ten system. Our results suggest that both Agilent and NimbleGen overall perform better than Illumina and that the high enrichment performance of Agilent is stable among samples and between vendors, whereas NimbleGen is only able to achieve vendor- and sample-specific best exome coverage. Moreover, the recent Agilent platform overall captures more coding exons with sufficient read depth than NimbleGen and Illumina. Due to considerable gaps in effective exome coverage, however, the three platforms cannot capture all known coding exons alone or in combination, requiring improvement. Our data emphasize the importance of evaluation of updated platform versions and suggest that enrichment-free WGS can overcome the limitations of WES in sufficiently covering coding exons, especially GC-rich regions, and in characterizing structural variants.
P-16: Evolution and clinical science: Still a long bridge to build

\author{
A. Khalique, ${ }^{1}$ S. Shakir, ${ }^{1}$ M. Qureshi, ${ }^{1}$ and $\underline{\text { M. Hussain }}{ }^{2}$ \\ ${ }^{1}$ Institute of Basic and Medical Sciences \\ ${ }^{2}$ Institute of Biological, Biochemical and Pharmaceutical \\ Sciences, Dow University of Health Sciences, Karachi, Pak- \\ istan \\ E-mail: mushtaq.hussain@duhs.edu.pk
}

Keywords: evolutionary medicine; basic sciences; clinical sciences

The aims and scope section of peer reviewed periodicals indicate the orientation of a journal to entertain a particular form of research. Here we explored through text mining the aims and scope of 2,061 journals published by eight leading publishers for the presence of evolutionary biology. The publishers included are Springer link, Wiley, Nature, Oxford, Elsevier, PLOS, BMC, and Bentham. Out of the assessed journals, only $244(\sim 12 \%)$ include the word evolution in their aims and scope and only 10 of those also count clinical investigations in their aims and scope. Only 22 journals have word evolution in their title and none of those except one mention clinical studies in their aims and scope. Similarly, journals mainly oriented to clinical and medical research account for nearly $50 \%$ of all the journals examined, and the word "evolution" was found missing in their aims and scope. We understand aims and scope of many reputable journals may be very generic and they often publish articles related to evolutionary biology. Nevertheless, the aims and scope is arguably the first indicator for the researcher to consider one's work for submission in a particular journal and may in turn encourage his or her current and/or future research endeavors. Considering this viewpoint, the present values do not seem encouraging and indicate a long gap to bridge between clinical and evolutionary biology research. Inclusion of evolutionary biology and clinical studies in the aims and scope of journals on both sides may help the cause and encourage the research activities linking evolutionary biology with clinical sciences.

P-17: The "French Disease": On the conceptual evolution of syphilis. The humanist Ulrich von Hutten (14881523) as an early syphilis patient

\section{U. L. Gantenbein}

Institute of Evolutionary Medicine, University of Zurich, Switzerland

E-mail: ursleo.gantenbein@iem.uzh.ch

Keywords: history of medicine; syphilis; paleopathology; early modern times

The early medical history of syphilis in Europe starts around 1495. The mysterious onset of a new chronic disease with various drastic symptoms lead already at an early stage 
to a tidal flood of publications and personal accounts on the subject. Among them we find the writings of probably the most prominent early syphilis patient, the German Imperial knight, humanist, and laureate poet Ulrich von Hutten (1488-1523). In his letters and his book "De Guaiaci Medicina et Morbo Gallico" (1519), he describes his views on the nature and cure of syphilis and the course of his own illness, thus transmitting invaluable first-hand information regarding the early history of syphilis. Already heavily marked with the late symptoms of syphilis, Hutten fled as a political refugee to Zurich and was assigned to the lonely Isle of Ufenau in the Lake of Zurich, where he died shortly after and was buried. In the course of times, the exact place of his grave was forgotten. In 1958, a team of archeologists thought they had excavated Hutten's skeleton, a supposition clearly rebutted by the Swiss anthropologist Erik Hug (1911-1991). Apparently stimulated by the subject, Hug was able to find and scientifically identify the authentic remains of Hutten some ten years later in 1968. Although Hug gave several talks on his important findings, he never publicized his results. After his retirement in 1980, Erik Hug consigned his entire scientific legacy regarding Hutten to the Monastery of Einsiedeln, who was the legal owner of the Isle of Ufenau since Hutten's times. Again forgotten until 2005, Hug's legacy is now cataloged and accessible in the archives of the monastery. The present talk covers aspects of the early history of syphilis and presents a preliminary paleopathological overview of the remains of Ulrich von Hutten based on Erik Hug's findings.

\section{P-18: Brazilian Baroque master exhumation for pale- opathological analysis}

T. Ferreira, ${ }^{1}$ R. Laguardia, ${ }^{2}$ and G. B. de Carvalho ${ }^{3}$

${ }^{1}$ Barbacena Faculty of Medicine, Brazil

${ }^{2}$ Radiology, Barbacena Faculty of Medicine, Brazil

${ }^{3}$ Dermatologist, Brazil

E-mail:trf299300@gmail.com

Keywords: paleopathology; Porphyria Cutanea Tarda; leprosy

Antônio Francisco Lisboa (1738-1814) is considered the greatest Baroque artist of Brazil. His works, considered as heritage of humanity, can be observed in the historic cities of Ouro Preto and Congonhas do Campo, in the State of Minas Gerais. There is a debate about the disease that led to his tragic death, involving multiple injuries in his limbs and face, which earned him the nickname of "Aleijadinho" (Little Cripple).

Bretas (1815-1866), his main biographer, reports that he was a healthy man until 47 years old when his body was affected by multiple skin lesions, leading to deformation in his face and limbs. Historical accounts linked the case to leprosy, due to facial and ulnar paralysis, and partial loss of limbs.
Carvalho (1999), after exhumation, conducted a pathological examination of Aleijadinho's bones. Those were measured and cataloged. Macroscopically, the reddish color, caused by bioaccumulation of iron ions, drew attention. Samples of thoracic vertebrae accrued rates of $340 \mu \mathrm{g} / \mathrm{g}$ of iron in the bones (reference value: $0,088 \mu \mathrm{g} / \mathrm{g}$ ). The poor state of the skeleton did not show any contributions to the leprosy frame.

Although the bones did not contribute to one diagnosis, it pointed to another possibility. High levels of iron in the bones are associated with Porphyria Cutanea Tarda but this frame can only be conclusive through DNA analyses, which have not been undertaken so far. If confirmed, Aleijadinho's will be the oldest case of Porphyria Cutanea Tarda in history.

P-19: Does quality of the childhood environment predict pubertal developmental trajectories? A test of life history models in a population of contemporary British adolescents

$\underline{\text { A. van Leeuwen and R. Mace }}$

University College London

E-mail: a.leeuwen@ucl.ac.uk

Keywords: puberty; life history theory; physical development; adolescence

Several well-known life history models of adolescent development predict that an unfavorable (e.g., resourcepoor) childhood environment will accelerate reproductive development and result in early puberty, which is in turn associated with an increased risk of a suite of somatic and mental health problems during adolescence and later in life.

We investigate whether measures of the quality of the childhood environment - such as father absence, quantity of direct parental care, household socioeconomic status, and area deprivation - are associated with multiple measures of pubertal development: menarche and breast development for girls; voice breaking and penis and scrotum development for boys; and pubic and armpit hair growth and the pubertal growth spurt for both boys and girls. To this end, we apply recently developed methods to derive developmental parameters to be used as dependent variables in our analyses. In particular, we use the SuperImposition by Translation and Rotation (SITAR) technique for summarizing height growth, and mixed effects models for pubertal development (Tanner) stages.

This study represents one of the most comprehensive tests of life history predictions about childhood environmental influences on pubertal timing. No previous study has looked at this many measures of adolescent physical development in the same population, in relation to life history theoretical predictions. Moreover, since boys have been severely understudied, it represents a particularly 
significant contribution to our understanding of male physical development from a life history perspective.

\section{P-20: Principles of evolutionary medicine in programs against malaria}

D. Ocampo

\section{Durham University, $U K$}

E-mail: denise.ocampo@durham.ac.uk

Keywords: evolution; malaria; global health

In the past, malaria eradication programs have failed, as it is still one of the infectious diseases with the highest death rates. It has been argued that malaria cannot be eradicated but only controlled. According to reports issued by the WHO, there has been a steady decline of malaria incidences and deaths. The important questions are is this only a short-term victory and will morbidity and mortality numbers shoot up again after the current attention and funding subsides?

The answer is most probably: yes. Research has shown that coevolution between parasites, vectors, and hosts have direct consequences on virulence and transmission. Human behavior is putting selective pressures on the vector and the pathogen via different pathways. Parasitic resistance towards treatments and behavioral changes in vectors due to human interventions such as bed nets are evolving faster than our counter actions. Knowledge on how parasites, vectors, and hosts coevolve gives us an advantage in the battle against malaria by considering what the most probable next step will be and how to plan protection effectively. We can predict changes and give warnings about future scenarios. In my dissertation, I will be asking; are evolutionary medicine theories being considered in the fight against malaria? Are they being put effectively into practice? Does academia merge with practical applications and can it be more efficient? I will therefore interview people working in current health programs, run by WHO, Roll Back Malaria and others, as well as in academic research. My main aim is to find out if evolutionary aspects of the diseases are considered important in combating malaria and if not, why not and should they be incorporated effectively into malaria programs?

P-21: Room for evolution in med-school? An online survey of medical students' opinion of evolutionary medicine in the UK

\section{A. Alvergne}

School of Anthropology, Oxford University, UK

E-mail: alexandra.alvergne@anthro.ox.ac.uk

Keywords: medical school; United Kingdom; teaching; social sciences

Background. The relevance of evolution for addressing medical issues is now well established among evolutionary scholars. However, the extent to which the medical profession shares this view is less clear. The few papers on the topic suggest that medics are likely to dismiss evolution as irrelevant to everyday practice and think that there is no need to teach evolution in medical school. Yet, no study has actually quantified the opinion of medical students as to whether or not they think they should be taught evolutionary theory and its application to medicine. Methods. To address this deficit, a survey targeting medical students in the UK and aiming at quantifying the knowledge and opinion of social sciences subjects associated with medical training (medical anthropology, evolutionary medicine, health psychology, and medical sociology) was created and published online. The survey was disseminated to both graduate and undergraduate medical students using mailing lists obtained from University offices. All UK medical schools were approached. Results. Preliminary results suggest that while medical students broadly know or can guess what evolutionary medicine is, less than half think that it should be part of their syllabus, which contrasts with their view on other disciplines such as health psychology. The poster will also include results on how opinion varies as a function of the year of study, sex, and medical speciality. It is predicted that those students who are most favorable to the teaching of evolutionary medicine are those wishing to specialize in areas where direct applications have already been demonstrated. Conclusion. Although the survey will be running until July 2015, preliminary results suggest that most medical students surveyed do not think that they should be taught evolutionary medicine. The reasons why this is the case will be discussed.

\section{P-22: Selection gradients and ecogeographic variance in the human postcrania}

K. Savell, ${ }^{1}$ C. Roseman, ${ }^{2}$ and B. M. Auerbach ${ }^{1}$

${ }^{I}$ Department of Anthropology, University of Tennessee Knoxville, Knoxville, USA

${ }^{2}$ Department of Anthropology, University of Illinois at Urbana-Champaign, Urbana, USA

E-mail: ksavell@vols.utk.edu

Keywords: natural selection; ecogeographic variation

Researchers use human body proportions to examine population relationships, effects of nutritional stress, and, overwhelmingly, evolutionary change in response to factors associated with latitude (i.e., ecogeography), such as parasite loads or climate. Recent work by Roseman and Auerbach (2015) indicates that the ecogeographic distribution of human body proportions is driven by a combination of population structure and natural selection. This contrasts with assumptions that selection alone produces morphological variance along ecogeographic clines (i.e., Bergmann's and Allen's “rules"). Since Roseman and 
Auerbach's (2015) approach does not distinguish between direct and indirect responses to selection acting on body proportions, this study retrospectively estimates selection gradients of limb lengths and body breadth to assess the nature of selective forces on ecogeographic morphological variance.

We estimated the vectors of selection gradients required to evolve one group into another for pairs of populations across major climate regions, and deconstructed resulting matrices to explore interelement effects. Results indicate that strong selection on distal limb lengths and body breadth effected an evolutionary transition from morphologies found in "tropically-adapted" Sub-Saharan Africa to those in the arctic. Similar, but weaker, patterns of selection were necessary to effect a transition in proportions from SubSaharan Africa to more temperate regions (i.e., Europe and North Africa). Evidence of differential response between elements was also noted: strong negative selection in tibial length at higher latitudes affected the ability for other long bones to response to selective pressure. We conclude that natural selection contributes to evolution in limb length and body breadth, though acting differentially across elements and with variation between populations. In addition, selection in a single element appears to affect changes elsewhere, emphasizing our need for a holistic, full-organism approach to studying human morphological variation.

\section{P-23: Body mass index and blood pressure: A compara- tive study between Nigerians and Ugandans}

M. G. Taura, L. H. Adamu, A. Gudaji, and M. H. Modibbo

Department of Anatomy, Faculty of Basic Medical Sciences, Bayero University, Kano, Nigeria

E-mail:mgtaura.ana@buk.edu.ng

Keywords: Africans; body mass index; blood pressure; marital status

The study aimed to assess gender differences within and between two African population groups (Nigerians and Ugandans). It also highlighted the role of marital status (MS) in evaluation of body mass index (BMI) and blood pressure (BP) among Nigerians and Ugandans. Association between sex and marital status with BMI and BP was investigated and compared between the two populations. A total of 287 Nigerians (205 males and 82 females) and 233 Ugandans (143 males and 90 females) mean age $25.66 \pm 5.45$ years participated. Standard procedures were employed in the measurement of height, weight, and BP. One-way analysis of variance and Chi square/Fisher's exact tests were used to determine differences and associations respectively $(P<.05)$. The result showed that for BMI, female Ugandans exhibit differences to male Nigerians, female Nigerians, and male
Ugandans. Similarly, female Nigerians and male Ugandans showed sex-based differences. Sexual dimorphism was observed in both diastolic blood pressure (DBP) and systolic blood pressure (SBP) among the Nigerian population. However, for SBP, a difference was also noticed in male Nigerians compared with both male and female Ugandans. With regards to the DBP, differences were observed with female Ugandans compared to male Ugandans and male Nigerians. In addition, male Ugandans and female Nigerians exhibit sexual dimorphism. All the three variables were found to be associated with sex among the two population groups with exception of BMI among Nigerians. With respect to MS, BMI showed differences between married and unmarried Nigerians, married and unmarried Ugandans. BP only SBP shows a difference between unmarried Nigerians and unmarried Ugandans. Only BMI was found to be associated with MS in Nigerian males and in both sexes among Ugandans. In conclusion, apart from sex, MS was also another factor that may bring about the differences.

\section{P-24: Mismatch to modernity: Heightened sensitivity in seasonal affective disorder (SAD)}

\section{P. Papino-Wood and $\underline{\mathrm{H}}$. Nasreen \\ New York Medical College, USA \\ E-mail: nasreen_haque@nymc.edu}

Keywords: SAD; depression; senses; mismatch

Seasonal affective disorder (SAD) is a form of depression that reoccurs yearly with varied symptoms experienced from one person to another, and severity falling on a very wide spectrum from unnoticeable to suicidal tendencies. Among the several high risk factors associated with SAD, female gender and/or geographical location seem to play a significant role. The rate of incidence rises in frequency in relation to the distance from the equator; the further away from the equator one lives, access to sunlight is reduced and availability of vitamin D levels becomes lower. This supports the current prevailing theory that SAD occurs more in the winter months, where the days are shorter and individuals are exposed to decreased levels of sunlight. Vitamin D or artificial sunlight exposure should then be enough to counter these effects and cure all cases of SAD. However, such therapies are not always curative. Perhaps the link to SAD may not be dependent on vitamin D absorption alone, which may be a byproduct of the mechanism of action. We propose that SAD is a trade-off to the previous benefit of enhanced sensitivity to light, sound and/or temperature which promoted fitness in the hunter-gatherer era. A genetic predisposition for these enhanced senses: adjusting to the dark faster, increasing body temperature, as well as increased alertness, would have increased fitness in the hunter-gatherer era. The trade-off would have been social withdrawal and decreased expenditure of energy, each 
with the benefits of allowing the body to rest and self-repair and avoiding conflict to live to see another day. If this is a survival mechanism inherited from the hunter-gatherer era, perhaps individuals who suffer from SAD suffer from overstimulation due to their increased sensitivity. New therapies may be developed utilizing desensitization techniques to help block out overstimulation in today's modern society.

P-25: A deviant, prone burial from Fraumünster, Zurich (early 17th century) and the evolution of rheumatoid arthritis

D. Moser, ${ }^{1}$ R. Seiler, ${ }^{2}$ K. Link, ${ }^{2}$ M. Bertschi, ${ }^{3}$ P. Ohnsorg, ${ }^{3}$ E. Langenegger, ${ }^{4}$ T. Boeni, ${ }^{2}$ and M. Haeusler ${ }^{2}$

${ }^{1}$ Institut für Archäologie, Fachbereich Prähistorische Archäologie, Universität Zürich, Switzerland

${ }^{2}$ Institut für Evolutionäre Medizin, Universität Zürich, Switzerland

${ }^{3}$ Archäologie, Hochbaudepartement der Stadt Zürich, Switzerland

${ }^{4}$ Anthropologisches Institut und Museum, Universität Zürich, Switzerland

E-mail:daria.moser@uzh.ch

Keywords: paleopathology; rheumatoid arthritis; periodontitis; early modern period

In 2014, a prone (i.e., face down) burial of a well-preserved skeleton of a 30-year-old man was discovered in the cemetery of the former Fraumünster monastery in Zurich, Switzerland. It is radiocarbon-dated to 1587-1621 AD \pm 27 years. The closely packed extremities suggest that the deceased was wrapped in a shroud with the legs perhaps tied together, and the arrangement of the pit indicates a certain degree of hastiness. Macroscopic, radiological, histological, and Micro-CT analyses reveal arthritic changes with pitting, hypervascularization, and hemosiderin deposition in the joints that symmetrically involve 19 joint regions while sparing the axial skeleton. This pattern is pathognomonic of early rheumatoid arthritis. The prevailing hypothesis for rheumatoid arthritis evolution proposes an American origin ca. $2000 \mathrm{BC}$ and dates the earliest unambiguous case in the Old World to 1800 AD. Accordingly, any presumed diagnosis of rheumatoid arthritis in earlier European archeological remains has been questioned due to bad preservation or uncharacteristic symptoms. The Fraumünster skeleton is therefore crucial in this debate, as it represents one of the strongest cases of rheumatoid arthritis in the early modern period. In addition, the skeleton shows signs of severe periodontitis, a disease recently associated with rheumatoid arthritis. The possible link is the periodontal pathogen Porphyromonas gingivalis, the only bacterium that citrullinates proteins. Autoimmune reaction against citrullinated proteins is essential in the pathogenesis of rheumatoid arthritis. Moreover, prone burials in Europe are often associated with fear of the dead including revenants, but also with marked signs of diseases with cutaneous manifestation. The early involvement of multiple joints in the Fraumünster skeleton suggests an aggressive form of rheumatoid arthritis with a high risk for extraarticular complications. A possible frightening involvement of the skin can therefore be hypothesized to have played an important motive for this deviant, prone burial of the young man from Fraumünster.

\section{P-26: Appendectomy is followed by increased risk of hyperuricemia}

\section{T. H. Chung}

Department of Family Medicine, College of Medicine, Ulsan University Hospital, University of Ulsan, South Korea

E-mail: jeongth@uuh.ulsan.kr

Keywords: appendectomy; hyperuricemia

Background. Some investigators have proposed that the human appendix is well suited as a "safe house" for commensal bacteria. Uric acid is the end product of the metabolism of purine compounds and it is eliminated by gut and the kidney. Intestinal tract bacteria degrade urate, the ionized forms of uric acid. This intestinal uricolysis is responsible for approximately one-fourth to one-third of total urate disposal. We hypothesized that the appendix might be involved in the physiology of intestinal uricolysis and appendectomy can decrease the intestinal uricolysis. As a result, appendectomy might cause hyperuricemia. Method. Data were obtained from 12,936 subjects who were at least 20 years old and who underwent a routine health checkup at the Ulsan University Hospital Heath Promotion Center in Ulsan, South Korea between March 2010 and February 2011. We compared the risk of hyperuricemia for the subjects who are appendectomized with that for the subjects who are not appendectomized. The odds ratios of hyperuricemia were calculated using a logistic regression model. Age, body mass index, alcohol intake, smoking, and exercise were included in the multivariate analysis. Results. There was a significant association between appendectomy and hyperuricemia (adjusted OR 1.39, 95\% CI 1.01-1.92). After reclassifying the subjects into three groups according to the duration since appendectomy, as $\leq 9$ years, 1019 years, and $\geq 20$ years, logistic regression analysis showed the dose response relationship between the duration since appendectomy and the risk of hyperuricemia. The relationship was still significant after adjusting for age, body mass index, alcohol consumption, smoking, and exercise (adjusted OR $0.91,95 \%$ CI $0.56-1.48,1.13,95 \%$ CI $0.73-1.75$, and 1.47, 95\% CI 1.47 1.07-2.01, resp.). Conclusion. Appendectomy was positively associated with hyperuricemia. The appendix might function in the 
physiology of intestinal uricolysis as a safe house for commensal bacteria.

\section{P-27: Sexually transmitted pathogens, depression, and premenstrual syndrome}

C. Doyle, ${ }^{1,2}$ W. Swain, ${ }^{3}$ H. Swain Ewald, ${ }^{1}$ C. Cook, ${ }^{4}$ and P. W. Ewald ${ }^{1}$

${ }^{1}$ Department of Biology, University of Louisville, Louisville, USA

${ }^{2}$ Department of Biology, Indiana University Southeast, New Albany, USA

${ }^{3}$ Peabody College of Education and Human Development, Vanderbilt University, Nashville, USA

${ }^{4}$ Department of Obstetrics, Gynecology \& Women's Health, University of Louisville, Louisville, USA

E-mail:doylecar@ius.edu

Keywords: chlamydia trachomatis; depression; PMS

This study investigated whether sexually transmitted infections were associated with depression and other debilitating manifestations of illness that are exacerbated perimenstrually, particularly depression, headaches, nausea, and pain. The following pathogens were investigated: human papillomavirus, Chlamydia trachomatis, Neisseria gonorrheae, Gardnerella vaginalis, Candida albicans, and Trichomonas vaginalis. Data were gathered from medical records of 500 regularly cycling women. C. trachomatis was significantly associated with premenstrual syndrome (PMS) and two common manifestations of PMS: depression and pain. T. vaginalis was significantly correlated with headache and $G$. vaginalis with nausea. None of the illness manifestations were significantly associated with the tested life-style variables: dietary calcium supplementation, alcohol and drug use, exercise, and smoking. Conclusions. These associations provide a basis for further assessment of infectious causation of depression and other manifestations of illness that are commonly associated with PMS; they raise the possibility that the frequency and severity of these manifestations might be reduced by treatment and prevention of infections.

\section{P-28: Influence of thoracolumbar transitional vertebra position on sagittal mobility}

$\underline{\text { N. Frater, }}{ }^{1}$ N. Bonneau, ${ }^{2}$ C. Tardieu, ${ }^{2}$ and M. Haeusler ${ }^{1}$

${ }^{1}$ Institute of Evolutionary Medicine, University of Zurich, Switzerland

${ }^{2}$ Muséum National d'Histoire Naturelle, Paris, France E-mail: nakita.frater@iem.uzh.ch

Keywords: hominid evolution; lordosis; spinal mobility; rotational stability

The evolution of bipedalism is often thought to have been facilitated by a longer "functional" lumbar portion of the vertebral column in early hominids compared to modern humans. The so-called functional definition of the lumbar spine is based on articulation types. In the majority of modern humans, the transition from ventrolaterally to dorsomedially oriented articular facets is at T12, thus coinciding with the last rib-bearing vertebra. Although fossil hominids show the same segmentation pattern as modern humans with the same number of rib-bearing and rib-free vertebrae, they all show a more cranial transition of the articulation types. The transitional vertebra is at T11 in KNM-WT 15000 (Homo erectus), MH2 (Australopithecus sediba), Sts 14 and Stw 431 (A. africanus).

Here, we test the hypothesis that a longer "functional" lumbar spine is related to higher capacity for flexion and extension. We analyzed mobility on functional lumbar radiographs of 120 patients with either unspecific low back pain, spondylolisthesis, or a history of disc herniation in the lower lumbar spine. These pathologies might disturb spinal mobility locally at the lumbosacral junction, but there was no indication that they affected mobility at the thoracolumbar border. We calculated sagittal mobility from the angles between the anterior borders of S1 and T11 as well as between L3 and T11 in maximal flexion and maximal extension.

We found no influence of the position of the transitional vertebra on flexion/extension mobility. Therefore, it seems unlikely that a cranial position of the transitional vertebra facilitated the evolution of bipedalism. Rather, it may be possible that a longer "functional" lumbar spine reflects an adaptation to climbing behavior. Lumbar like articulations mainly provide rotational stability of the trunk skeleton, which is favorable in climbing, yet relatively disadvantageous in running and hence might have been counter selected in later Homo.

P-29: Relationship of rotator cuff lesions of the human shoulder joint to the evolution of bipedalism reanalyzed

$\underline{\text { S. Mathews }}{ }^{1}$ P. Schmid, ${ }^{2,3}$ and M. Haeusler ${ }^{1}$

${ }^{1}$ Institute of Evolutionary Medicine, University of Zurich, Zurich, Switzerland

${ }^{2}$ Anthropological Institute and Museum, University of Zurich, Switzerland

${ }^{3}$ Evolutionary Studies Institute, University of the Witwatersrand, South Africa

E-mail: sandra.mathews@iem.uzh.ch

Keywords: shoulder pathologies; impingement syndrome; subacromial space; 3D geometric morphometric

The morphology of the shoulder girdle not only plays an important role in the discussion of the locomotor behavior of early hominins, but it may also be an etiological factor in degenerative shoulder disorders that mainly includes rotator cuff disease or shoulder impingement syndrome. 
Yet, shoulder pathologies are virtually absent in great apes that habitually use their arms in overhead activities during climbing, even taking age into account. Specifically, a relatively smaller subacromial space in modern humans is thought to facilitate compression of the supraspinatus tendon and bursa, while a smaller supraspinatus muscle is hypothesized to lead to dysbalance of the rotator cuff muscles and overloading of the supraspinatus tendon, thus contributing to the development of shoulder impingement syndrome. However, previous studies of the threedimensional structures of the shoulder girdle were based on linear measurements and indices or two-dimensional radiographs and MRI sections only.

Here, we perform a three-dimensional landmarkbased geometric morphometric analysis of the scapular morphology in extant great apes, including Hylobates lar $(N=71)$, Pongo sp. $(N=20)$, Gorilla gorilla $(N=39)$, Pan troglodytes $(N=44), \mathrm{MH} 2$ (Australopithecus sediba), KNM-WT 15000 (Homo erectus) and a sample of modern humans (Homo sapiens, $N=89$ ). We show that the supraspinous fossa and by inference the supraspinatus muscle have the same size in all examined species relative to the size of the upper extremity. Also the subacromial space has a virtually identical relative size in great apes and humans. This challenges common theories about the pathophysiology of shoulder impingement syndrome. We conclude that the etiology of subacromial impingement syndrome is probably not related to the reorganization of the shoulder morphology during the evolution bipedalism. Moreover, our study demonstrates that linear measurements and indices do not acknowledge the complex 3D anatomy of the shoulder girdle.

P-30: PCR methods to trace malaria indirectly by looking at G6PD variants in archeological specimens

C. Viganó, ${ }^{1}$ F. Rühli, ${ }^{1}$ K. Shimizu, ${ }^{2}$ and A. Bouwman ${ }^{1}$

${ }^{1}$ Institute of Evolutionary Medicine, University of Zurich, Switzerland

${ }^{2}$ Institute of Evolutionary Biology, University of Zurich, Switzerland

E-mail: claudia.vigano@iem.uzh.ch

Keywords: ancient DNA; G6PD deficiency; malaria

The presence of malaria in ancient Europe is well documented in historical records, however Plasmodium $s p$. DNA was successfully detected only in very few studies on ancient European samples (Sallares 2001). The concentrations of the parasite DNA preserved in human bones after death are often below the limit of the detection method (Taylor 1997), therefore is not surprising if it turned out to be impossible to detect many cases of malaria in the past. A more promising approach is to trace the presence of malaria in a population indirectly, by looking for human genetic mutations, such as G6PD deficiency, that have appeared in past in some populations as a result of natural selection by malaria. The recovery of these mutations in a skeleton would not proof that that individual had been infected with malaria but it would indicate a history of endemic malaria in that population. In this study a PCR method to detect G6PD A- and G6PD Mediterranean, the most common variants in Europe, were developed. The method was designed to amplify small G6PD gene fragments in archeological specimens. The methods were first tested on modern DNA and then applied to 20 Medieval teeth from different individuals buried in Corsica, a Mediterranean island with a history of endemic malaria. Amplicons were obtained from all samples allowing sequencing of the gene fragments to identify the target alleles.

\section{P-31: Optimization of DNA extraction protocols from historic formalin-fixed soft tissues for retrospective molecular analyses}

G. Ferrari, ${ }^{1}$ G. Akgül, ${ }^{1}$ F. Rühli, ${ }^{1}$ K. Shimizu, ${ }^{2}$ and A. Bouwman $^{1}$

${ }^{1}$ Institute of Evolutionary Medicine, University of Zurich, Switzerland

${ }^{2}$ Institute of Evolutionary Biology, University of Zurich, Switzerland

E-mail: giada.ferrari@iem.uzh.ch

Keywords: ancient DNA; archival formalin-fixed tissues; historic pathogen genomes

Retrospective molecular studies allow researchers to reconstruct the epidemiology of infectious diseases in the past, and therefore play a fundamental role in the study of the evolutionary genetics of pathogens, immunity and hostpathogen coevolution. Understanding the epidemiology, host-pathogen interactions of past infections and how virulence has evolved, is of particular relevance in the context of the emergence and re-emergence of infectious diseases. Formalin-fixed wet specimens from museums and pathologic-anatomical collections represent an extensive and disease-specific archive for retrospective molecular investigations. Their precise dating and available diagnoses make them an ideal source for the reconstruction of historic human and pathogen genomes. However, while formalin fixation successfully preserves tissue integrity, it degrades DNA into short fragments, similar to that with ancient DNA. Furthermore, formaldehyde creates cross-links between nucleic acids and proteins, which results in DNA being unavailable for downstream applications. Protocols that apply high thermal energy during tissue lysis to reverse cross-linking have been reported to successfully isolate DNA from formalin-fixed tissues. However, this results in a trade-off between cross-link reversal and further DNA fragmentation. 
The aim of this study is to develop a methodology to obtain DNA suitable for high-throughput sequencing from archival formalin-fixed tissues while balancing cross-link reversal and thermal-induced DNA fragmentation. To this end we evaluated ten protocols for DNA extraction from 19th and early 20th century museum formalin-fixed wet specimens obtained from individuals that suffered from tuberculosis, leprosy or anthrax. Quantitative PCR assays, as well as length, quality and mappability to the human and pathogen reference genomes of Illumina shot-gun reads are used to evaluate the different extraction protocols. By optimizing the quality of DNA than can be obtained from these specimens, it is possible to unlock the potential of formalin-fixed archival soft tissues for retrospective molecular studies.

\section{P-32: The periodontitis-arteriosclerosis syndrome: Another aspect of arteriosclerosis an Ancient Egypt}

\section{R. Seiler and F. Rühli}

Institute of Evolutionary Medicine, Medical Faculty, University of Zurich, Switzerland

E-mail: roger.seiler@iem.uzh.ch

Keywords: radiology

"Knowledge of the heart" from the Papyrus Ebers (1555 $\mathrm{BCE})$ is the earliest known text on cardiology. Here the heart is described as the organic motor of the body. But in ancient Egypt the heart also has an important meaning as the center of the body and the soul. Thus, it had to be left in the body during mummification, but it is therefore subject to severe alterations. On the other hand, the great vessels, for example in the retroperitoneal space or in the extremities, can be better preserved. As a consequence, their pathological changes are often visible and have been the object of paleopathological investigations since the 19th century, first by dissection (Ruffer 1911), and more recently by computed tomography (Allam et al. 2009), and arterial calcifications which are pathognomonic for atherosclerosis (Lim et al. 2008) have been observed. Ruffer stated, that "such lesions were as [...] ago as they are today" and recently Thompson diagnosed atherosclerosis in 38\% of ancient Egyptians mummies (Thompson et al. 2013). Ruffer could not "give any reason why arterial disease should have been so prevalent in ancient Egypt". For David (2013) the reason was "almost certainly [...] a diet rich in saturated fat that was confined to the elite," and Thomas (Thomas et al. 2014) named as "potential causes [...] frequent and chronic infections resulting in chronic inflammation, smoke inhalation, or other as yet undiscovered risk factors." Surprisingly, the most common type of infection in mankind? oral infection? is not mentioned. During the past years the knowledge of the likely interaction of periodontal disease and arteriosclerosis, their common predisposing factors and of the etiologic mechanisms has grown constantly (Kohly et al. 2015). So the presentation of a two mummy case study on the basis of detailed radiological analysis can shed new light on arteriosclerosis in ancient Egypt.

P-33: Anthropological-paleopathological investigations in tomb KV40 in the Kings' Valley (Western Thebes, Upper Egypt)

S. Meyer, ${ }^{1}$ N. Frater, ${ }^{1}$ R. Seiler, ${ }^{1}$ Öhrström L, ${ }^{1}$ S. Bickel, ${ }^{2}$ and F. Rühli ${ }^{1}$

${ }^{1}$ Institute of Evolutionary Medicine, University of Zurich, Switzerland

${ }^{2}$ Antiquity Department, University of Basel, Switzerland

E-mail: sabrina.meyer@iem.uzh.ch

Keywords: mummies; disarticulated human remains; stress markers; developmental abnormalities

In this study we present the anthropological and paleopathological findings of the human remains from tomb KV40 in the Kings' Valley in Upper Egypt. These preliminary findings were collected during the field season of 2015. The tomb was first accessed in 2011 by the University of Basel where the archeological findings showed an initial burial period dating to the 18th dynasty (ca. 1479-1350 BC), followed by reuse of the tomb in the Third Intermediate Period (ca. 1077-800 BC). It has also been subject to tomb robberies in ancient times. These disturbances led to disintegration, disarticulation and scattering of the mummified remains. In addition, a fire occurred in the late 19 th century AD, which caused even more damage, leaving burnt human remains in every stage of fragmentation.

In the first anthropological field season the human material was catalogued and the minimal number of individuals determined. Further investigations into the sex and age distribution, and body height and paleopathological alterations are ongoing. So far the human material has been catalogued and photo-documented, followed by radiological imaging (portable X-ray generator EXAMION PX60HF) of the bestpreserved mummified and pathologically altered remains.

Currently, the minimum number of individuals is sixty. Both sexes are present within the adult sample although the proportion has not yet been determined. All age ranges from perinatal to late adulthood are represented. There are several cases of paleopathological interest in KV40. Developmental abnormalities (for example a possible case of Achondroplasia) were found, as well as a case of skullcap deformation and long-bone fractures. In addition, several stress markers (e.g., Harris lines, cribra orbitalia) indicate poor health in some individuals.

Additional field seasons will follow to shed more light on these special burials. 
P-34: Risk of CT scans to the ancient DNA in dry, wet, and frozen states: A comparative Geant4-DNA study

\section{J. Wanek and F. Rühli}

Institute of Evolutionary Medicine, University of Zurich, Switzerland

\section{E-mail:johann.wanek@iem.uzh.ch}

Keywords: computed tomography; ancient DNA; risk; Geant4-DNA

When ancient remains undergo a computed tomography (CT) examination for the investigation of diseases in mummified bodies, ancient DNA (aDNA) fragments may become damaged. When this happens, it can subsequently reduce the possibility of DNA amplification following ionizing radiation. Our previous Monte Carlo (MC) simulation confirmed the expectation that the radiosensitivity of dry DNA is inversely proportional to the number of DNA fragments. However, the indirect effects of CT exposure on wet ancient cells have not been quantitatively examined in the past and may be important for subsequent genetic analysis. To remedy this shortcoming, cell models were developed using the new MC-code from the European Space Agency (ESA) called Geant4-DNA.

Cell hits were simulated using electrons with different energies, namely $5 \mathrm{keV}, 10 \mathrm{keV}, 15 \mathrm{keV}$, and $20 \mathrm{keV}$. The produced chemical species around the electron track forms a volume inside the water phantom called volume of risk (VOR). By calculation of the DNA fragment density from AFM images (taken from the literature), the radiation effects on wet DNA fragments at $25^{\circ} \mathrm{C}$ were assessed. Note that the radiation effects on cells at $0{ }^{\circ} \mathrm{C}$ were estimated employing the Einstein-Stokes and Einstein-Smoluchowski relations.

It is concluded that the probability of aDNA damage following CT imaging depends on the DNA fragment density and distribution. Other important factors were the absorbed energy and the temperature of wet cells. The indirect radiation damage to fragmented DNA in aqueous solution was summarized using the following probability ranges: at $25^{\circ} \mathrm{C}:\left[2 \times 10^{-7} ; 9 \times 10^{-7}\right]$; at $0^{\circ} \mathrm{C}\left[0.9 \times 10^{-7}\right.$; $\left.4 \times 10^{-7}\right]$. Here we present new evidence that the risk of aDNA damage drops about three orders of magnitude compared to normal DNA $\left(P \approx 1 \times 10^{-4}\right)$, when ancient cells are in wet or frozen state. Moreover, reducing the temperature reduces the radiation risk of aDNA damage significantly.

\section{P-35: The Canopic Jar Project_preliminary results}

M. Habicht, A. Bouwman, K. Link, and F. Rühli

Institute of Evolutionary Medicine, University of Zurich, Switzerland

E-mail:michael.habicht@iem.uzh.ch
Keywords: canopics; ancient DNA; histology; imaging techniques

Whilst ancient Egyptian mummies have been the main focus of research for centuries, ancient Egyptian canopic jars, with their intestinal contents, have been widely neglected as a source of medical and Egyptological information. The aim of the Canopic Jar Project is to establish novel research procedures and to examine a larger series of ancient Egyptian human soft tissues samples in a truly interdisciplinary research setting (medical, genetic, chemical and Egyptological). In the current pilot phase of the project, canopics from different time periods were studied (Burgdorf. Völkerkundemuseum BU_53_07668. Unknown owner, Late Period; Munich, SMÄK, ÄS 0026. Unknown owner, Late Period, St. Gallen, Stiftsbibliothek. Viscera bundles of Shep-enIsis, Late Period; Turin, Museo Egizio Cat. 3459A and $\mathrm{C}$, New Kingdom). The canopic jars have been analyzed Egyptologically, radiologically (by portable Xray, Examion PX $60 \mathrm{HF}$; or by computed tomography, Somatom Definition Flash), and by standard histological techniques such as Hematoxylin and Eosin or MassonGoldner staining. Mitochondrial DNA was successfully amplified, cloned, and sequenced in a dedicated aDNA facility at the Institute of Evolutionary Medicine, University of Zurich. All sequences were consistent with a westernEurasian/northern-African maternal ancestry. The samples also underwent probe based real-time PCR amplification to identify sex-specific amelogenin polymorphisms. In addition, samples underwent high-throughput sequencing (Illumina HiSeq 2500 v4 sequencing). Some pathogenic species were found (e.g., MTBC and Leishmania infantum). Samples were also investigated by gas-chromatographymass spectrometry and liquid chromatography-high resolution mass spectrometry. The extracts from different canopics showed different chemical compositions, and different (methylated) fatty acids were also identified. The Canopic Jar Project is a novel, truly interdisciplinary approach adding to already established ancient mummy research, focusing on the separately preserved viscera.

Acknowledgments Funding (as of June 2015): University of Zurich (Einrichtungskredit F. Rühli), Mäxi Foundation, Life Science Zurich Business Network, Canton of Zurich - Department for Economic Affairs and Office for Economy and Labour, Roche and Novartis (Kontaktgruppe für Forschungsfragen), Swiss National Science Foundation SNSF, Swiss Academy of Medical Sciences SAMW, The city and canton of Zurich, and Theiler-Haag Foundation.

Administrative Support: Sandra Matthews and Marianne Ott from the Institute of Evolutionary Medicine. Proofreading: Abigail Bouwman (Institute of Evolutionary Medicine).

Abstract peer-reviewing by the other members of the Scientific Committee: Paul W. Ewald (President, University of Louisville, USA), Barry Bogin (Loughborough University, GBR), Martin Brüne (Ruhr Uni Bochum, GER), Jane E. Buikstra (Arizona State University, USA), Sir Peter Gluckman (University of Auckland, NZ), Bernard 
Harris (University of Strathclyde, GBR), Israel Hershkovitz (Tel Aviv University, ISR), Rick Maizels (University of Edinburgh, GBR), F. DeWolfe Miller (University of Hawaii, USA), Frank Rühli (University of Zurich, SUI), Frederic Thomas (University of Montpellier CNRS, FRA), Janina Tutkuviene (Vilnius University, LTU), Noreen Tuross (Harvard University, USA), Elizabeth W. Uhl (University of Georgia, USA), Jonathan Wells (UCL, GBR), Albert Zink (European Academy of Bolzano, ITA).

Conflict of interest The authors declare that they have no conflict of interest.

\section{Index}

\begin{tabular}{|c|c|c|c|}
\hline Adamu, L. H. & $\mathrm{P}-23$ & Doyle, C. & P-27 \\
\hline Akgül, G. & $\mathrm{P}-31$ & Dyble, M. & O-29 \\
\hline Aktipis, A. & $\mathrm{O}-47$ & Egger, M. & O-39 \\
\hline Althaus, C. & $\mathrm{O}-23$ & Eisenach, $\mathrm{K}$. & O-21 \\
\hline Alvergne, A. & $\mathrm{P}-21$ & Emery Thompson, M. & O-35 \\
\hline Andersen, $\mathrm{P}$. & O-05 & Escasa-Dorne, M. & $\mathrm{O}-13$ \\
\hline Arnal, A. & $\mathrm{O}-12, \mathrm{O}-25$ & Ewald, P. W. & $\mathrm{K}-01, \mathrm{P}-27$ \\
\hline Auerbach, B. M. & $\mathrm{O}-44, \mathrm{P}-22$ & Feinman, R. & O-50 \\
\hline Bacalini, M. & $\mathrm{O}-28$ & Felger, I. & $\mathrm{P}-05$ \\
\hline Baines, J. F. & O-27 & Ferrari, G. & $\mathrm{P}-31$ \\
\hline Balaskas, T. & O-05 & Ferreira, $\mathrm{T}$. & P-18 \\
\hline Barry, A. & $\mathrm{P}-05$ & Fingerhuth, S. & $\mathrm{O}-23$ \\
\hline Beheim, B. & O-35 & Floris, J. & O-31, P-09 \\
\hline Belov, K. & $\mathrm{O}-22$ & Fode, P. & $\mathrm{O}-05$ \\
\hline Bender, B. & O-39 & Foley, A. & $\mathrm{O}-45$ \\
\hline Bender, $\mathrm{N}$. & O-55, O-56 & Forni, D. & $\mathrm{O}-05$ \\
\hline Bentley, G. & K-05 & Franceschi, C. & $\mathrm{O}-28$ \\
\hline Bertschi, M. & $\mathrm{P}-25$ & Francken, M. & P-07 \\
\hline Best, R. & O-04 & Frater, N. & O-58, P-28, P-33 \\
\hline Bickel, S. & P-33 & Gagneux, $\mathrm{S}$. & O-21 \\
\hline Blackwell, A. & O-35 & Gantenbein, U. L. & P-17 \\
\hline Blyth, M. & O-52 & Garagnani, $\mathrm{P}$. & $\mathrm{O}-28$ \\
\hline Bock, M. & P-11 & Garcia Gomez, J. & $\mathrm{O}-02$ \\
\hline Bodas, A. & $\mathrm{P}-12$ & Geiger, M. & O-30, P-08 \\
\hline Boeni, T. & $\mathrm{P}-25$ & Giuliani, C. & $\mathrm{O}-28$ \\
\hline Bogaard, A. & P-05 & González Candelas, F. & O-53 \\
\hline Bogdanova, A. & O-10 & Grabliauskaite, K. & O-06 \\
\hline Bonhoeffer, S, & $\mathrm{O}-23$ & Graf, R. & O-06 \\
\hline Bonhomme, F. & $\mathrm{O}-12$ & Graham, A. & $\mathrm{O}-03$ \\
\hline Böni, T. & O-58 & Granato, E. & O-17 \\
\hline Bonneau, N. & P-28 & Grassberger, M. & $\mathrm{O}-48$ \\
\hline Boom, H. & O-21 & Grassl, G. & $\mathrm{O}-27$ \\
\hline Borgia, A. & O-04 & Greenspan, N. S. & $\mathrm{O}-14, \mathrm{O}-15$ \\
\hline Borgia, M. & O-04 & Gudaji, A. & P-23 \\
\hline Bosch, E. & $\mathrm{O}-24$ & Gurven, $\mathrm{M}$. & O-35 \\
\hline Bouwman, A. & $\begin{array}{l}\text { O-57, P-30, } \\
\text { P-31, P-35 }\end{array}$ & Habicht, M. & $\mathrm{P}-35$ \\
\hline Brites, D. & O-21 & Haeusler, M. & $\begin{array}{l}\mathrm{P}-25, \mathrm{P}-28, \\
\mathrm{P}-29, \mathrm{O}-58\end{array}$ \\
\hline Brown, $\mathrm{C}$. & O-50 & Hains, D. & $\mathrm{O}-05$ \\
\hline Brown, S. & O-08, O-11 & Hangartner, R. & O-58 \\
\hline Bruggmann, R. & P-15 & Harris, B. & O-49 \\
\hline Burger, $\mathrm{O}$. & O-01 & Harrison, A. & $\mathrm{P}-05$ \\
\hline Carrel, T. & P-15 & Harvati, K. & P-07 \\
\hline Carroll, M. & O-47 & Haussman, S. & P-08 \\
\hline Casali, A. & $\mathrm{O}-25$ & Henneberg, M. & $\mathrm{K}-04, \mathrm{O}-56$ \\
\hline Chatterjee, A. & $\mathrm{P}-13$ & Hershkovitz, I. & $\mathrm{O}-37$ \\
\hline Chaudhary, N. & O-29 & Hiller, M. & O-52 \\
\hline Chawla, S. & P-14 & Hinde, A. & O-49 \\
\hline Chung, T. H. & P-26 & Hollox, E. & $\mathrm{O}-05$ \\
\hline Claidière, N. & P-06 & Huellein, $\mathrm{T}$. & P-01 \\
\hline Clavien, P. A. & O-06 & Humar, B. & O-06 \\
\hline Collins Cook, D. & $\mathrm{O}-45$ & Humar, R. & O-06 \\
\hline Cook, C. & $\mathrm{P}-27$ & Hussain, M. & P-16 \\
\hline de Carvalho, G. B. & P-18 & Jaeggi, A. & $\mathrm{O}-35$ \\
\hline DeWolfe Miller, F. & O-51 & Jang, J. & O-06 \\
\hline Diwekar-Joshi, M. & P-12, P-13, P-14 & Jaqueline, C. & $\mathrm{O}-25$ \\
\hline
\end{tabular}

\begin{tabular}{|c|c|c|c|}
\hline Jarrell, J. & $\mathrm{O}-34$ & Öhrström, L. & P-11, P-33 \\
\hline Johns, S. & $\mathrm{O}-01$ & Okoniewski, M. & $\mathrm{P}-15$ \\
\hline Johnstone, R. & O-19 & Özen, A. & $\mathrm{P}-11$ \\
\hline Joloba, M. & $\mathrm{O}-21$ & Pagano, A. & O-50 \\
\hline Kaplan, H. & $\mathrm{O}-35$ & Page, A. & O-18, O-29 \\
\hline Keller, I. & $\mathrm{P}-15$ & Palfi, G. & $\mathrm{O}-46$ \\
\hline Khalique, A. & P-16 & Papenfuss, A. & $\mathrm{O}-22$ \\
\hline Koepfli, C. & $\mathrm{P}-05$ & Papino-Wood, P. & P-24 \\
\hline Kowarsky, M. & $\mathrm{O}-22$ & Passarino, G. & $\mathrm{O}-28$ \\
\hline Krüttli, A. & O-57 & Patil, P. & P-13 \\
\hline Kulkarni, S. & $\mathrm{P}-14$ & Patrignani, A. & P-15 \\
\hline Kumar, S. & O-36 & Pearse, A. M. & $\mathrm{O}-22$ \\
\hline Kümmerli, R. & $\mathrm{O}-08, \mathrm{O}-11, \mathrm{O}-17$ & Pilz, M. & $\mathrm{O}-48$ \\
\hline Laguardia, R. & P-18 & Pirazzini, C. & O-28 \\
\hline Langenegger, E. & P-25 & Polley, S. & $\mathrm{O}-05$ \\
\hline Laplane, L. & $\mathrm{O}-12$ & Posa, A. & $\mathrm{O}-46$ \\
\hline Lehmann, K. & O-06 & Postma, E. & O-07 \\
\hline Lewis, J. & O-29 & Poveda, L. & P-04 \\
\hline Limani, P. & O-06 & Prescott, N. & $\mathrm{O}-05$ \\
\hline Lindsey, E. & O-52 & Puche Gil, J. & $\mathrm{O}-02$ \\
\hline Link, K. & P-25, P-35 & Pujol, P. & $\mathrm{O}-12$ \\
\hline Linnenbrink, M. & $\mathrm{O}-27$ & Pund, A. & P-14 \\
\hline Loesch, S. & O-46 & Qureshi, M. & P-16 \\
\hline Louzada, S. & $\mathrm{O}-05$ & Radich, J. & $\mathrm{O}-47$ \\
\hline Low, $\mathrm{N}$. & $\mathrm{O}-23$ & Raijmakers, L. & P-05 \\
\hline Loynes, R. & $\mathrm{P}-03$ & Raptis, D. & O-06 \\
\hline Ludin, $\mathrm{K}$. & P-15 & Rattei, T. & O-46 \\
\hline Ludwig, U. & $\mathrm{P}-11$ & Rausch, P. & $\mathrm{O}-27$ \\
\hline Luiselli, D. & $\mathrm{O}-28$ & Reinhard, K. & $\mathrm{O}-42$ \\
\hline Mace, R. & K-07, O-29, P-19 & Renaud, F. & O-12 \\
\hline Maixner, F. & $\mathrm{O}-46$ & Rickenbacher, A. & O-06 \\
\hline Makhro, A. & $\mathrm{O}-10$ & Rimoldi, S. & O-39 \\
\hline Maley, C. & $\mathrm{O}-47$ & Roche, B. & $\mathrm{O}-12, \mathrm{O}-25$ \\
\hline Mansfield, J. & $\mathrm{O}-05$ & Rodrigues, A. & O-19, O-33 \\
\hline Mari, D. & $\mathrm{O}-28$ & Rodrigues, $\mathrm{P}$. & $\mathrm{P}-05$ \\
\hline Markell, M. & O-50 & Rodriguez, J. & $\mathrm{O}-09, \mathrm{O}-24$ \\
\hline Márquez, S. & O-50 & Roseman, C. & P-22 \\
\hline Mathew, C. & $\mathrm{O}-05$ & Ross-Gillespie, A. & $\mathrm{O}-08, \mathrm{O}-11$ \\
\hline Mathews, S. & P-29 & Röthlisberger, B. & $\mathrm{P}-15$ \\
\hline Matyas, G. & $\mathrm{P}-15$ & Rühli, F. & $\begin{array}{l}\text { O-55, O-56, O-57, } \\
\text { O-58, P-10, P-11, } \\
\text { P-30, P-31, P-32, } \\
\text { P-33, P-34, P-35 }\end{array}$ \\
\hline May, H. & $\mathrm{O}-37, \mathrm{O}-38$ & Salali, G. & O-29 \\
\hline McNary, J. & O-52 & Sánchez-Villagra, M. & $\mathrm{O}-30, \mathrm{P}-08$ \\
\hline Meienberg, J. & $\mathrm{P}-15$ & Saniotis, A. & O-56 \\
\hline Mercier, $\mathrm{H}$. & $\mathrm{P}-06$ & Satsangi, J. & $\mathrm{O}-05$ \\
\hline Mery, F. & $\mathrm{O}-25$ & Savell, K. & $\mathrm{P}-22$ \\
\hline Meyer, S. & P-33 & Sazzini, M. & $\mathrm{O}-28$ \\
\hline Migliano, A. B. & O-29 & Scherf, H. & P-07 \\
\hline Minder, C. & O-58 & Scherrer, U. & O-39 \\
\hline Minnikin, D. E. & $\mathrm{O}-16$ & Schlapbach, R. & $\mathrm{P}-04, \mathrm{P}-15$ \\
\hline Miton, $\mathrm{H}$. & $\mathrm{P}-06$ & Schmid, P. & P-29 \\
\hline Modibbo, M. H. & $\mathrm{P}-23$ & Schmutz, B. & $\mathrm{P}-10$ \\
\hline Molnar, E. & $\mathrm{O}-46$ & Schneider, R. & $\mathrm{O}-30$ \\
\hline Moreno, C. & $\mathrm{O}-25$ & Schuetz, F. & $\mathrm{P}-10$ \\
\hline Morgan, J. & $\mathrm{O}-26$ & Schuetz, M. & $\mathrm{P}-10$ \\
\hline Moser, D. & $\mathrm{P}-25$ & Schuler, B. & $\mathrm{O}-04$ \\
\hline Mueller, I. & $\mathrm{P}-05$ & Seiler, R. & $\mathrm{P}-25, \mathrm{P}-32, \mathrm{P}-33$ \\
\hline Munkholm, P. & $\mathrm{O}-05$ & Selevsek, N. & P-04 \\
\hline Myers, S. & $\mathrm{O}-01$ & Shakir, S. & P-16 \\
\hline Nanni, P. & $\mathrm{P}-04$ & Shimizu, K. & P-30, P-31 \\
\hline Nasreen, $\mathrm{H}$. & $\mathrm{P}-24$ & Sironi, M. & O-05 \\
\hline Navarro, A. & $\mathrm{O}-09, \mathrm{O}-24$ & Smith, D. & O-29 \\
\hline Nedelcu, A. & $\mathrm{O}-47$ & Solary, E. & $\mathrm{O}-12$ \\
\hline Nesse, R. & $\mathrm{K}-02$ & Soranno, A. & $\mathrm{O}-04$ \\
\hline Nettels, D. & $\mathrm{O}-04$ & Soria, R. & O-39 \\
\hline Nimmo, E. & $\mathrm{O}-05$ & Spataro, N. & $\mathrm{O}-24$ \\
\hline Nunney, L. & $\mathrm{O}-12$ & Sprouffske, K. & O-47 \\
\hline Oberkofler, C. & O-06 & Staub, K. & $\mathrm{O}-31, \mathrm{O}-55, \mathrm{P}-09$ \\
\hline Ocampo, D. & $\mathrm{P}-20$ & Stearns, S. & K-08 \\
\hline Ohnsorg, P. & P-25 & Stein, D. & $\mathrm{O}-37$ \\
\hline
\end{tabular}




$\begin{array}{llll}\text { Steinmann, B. } & \text { P-15 } & \text { Viganó, C. } & \text { P-30 } \\ \text { Stieglitz, J. } & \text { O-35 } & \text { Vind, I. } & \text { O-05 } \\ \text { Swain, W. } & \text { P-27 } & \text { Vinicius, L. } & \text { O-29 } \\ \text { Swain Ewald, H. } & \text { P-27 } & \text { Vinodbhai, P. } & \text { P-02 } \\ \text { Tardieu, C. } & \text { P-28 } & \text { Vittecoq, M. } & \text { O-12 } \\ \text { Tasiemski, A. } & \text { O-12 } & \text { von Rueden, C. } & \text { O-35 } \\ \text { Taura, M. G. } & \text { P-23 } & \text { Wahl, J. } & \text { P-07 } \\ \text { Taylor, R. } & \text { O-22 } & \text { Waldvogel, D. } & \text { O-07 } \\ \text { Thomas, F. } & \text { O-12, O-25 } & \text { Wampande, E. } & \text { O-21 } \\ \text { Thompson, J. } & \text { O-29 } & \text { Wanek, J. } & \text { P-34 } \\ \text { Tissot, T. } & \text { O-12 } & \text { Warinner, C. } & \text { O-57 } \\ \text { Trescastro Lopez, E. } & \text { O-02 } & \text { Warner, N. J. } & \text { O-41 } \\ \text { Trevathan, W. } & \text { K-06 } & \text { Warren, W. } & \text { O-22 } \\ \text { Trumble, B. } & \text { O-35 } & \text { Watve, M. } & \text { O-20, P-12, P-13, P-14 } \\ \text { Tuarev, D. } & \text { O-46 } & \text { Weigert, M. } & \text { O-08, O-11 } \\ \text { Tutkuviene, J. } & \text { O-43 } & \text { Wells, J. } & \text { O-19, O-32 } \\ \text { Uggla, C. } & \text { K-07 } & \text { Woitek, U. } & \text { O-31, P-09 } \\ \text { Uhl, E. } & \text { O-40 } & \text { Wong, E. } & \text { O-22 } \\ \text { Ujvari, B. } & \text { O-12 } & \text { Wu, C. } & \text { O-22 } \\ \text { Ungethuem, U. } & \text { O-06 } & \text { Wunderlich, B. } & \text { O-04 } \\ \text { Uvjari, B. } & \text { O-22 } & \text { Xu, Z. } & \text { P-15 } \\ \text { Vallier, M. } & \text { O-27 } & \text { Yang, F. } & \text { O-05 } \\ \text { van Leeuwen, A. } & \text { P-19 } & \text { Yian, Y. } & \text { O-06 } \\ \text { van Lueewen, B. } & \text { K-07 } & \text { Zerjavic, K. } & \text { P-15 } \\ \text { van Schaik, K. } & \text { O-54 } & \text { Zink, A. } & \text { O-46 } \\ \text { Veal, C. } & \text { O-05 } & \text { zur Hausen, H. } & \text { K-03 } \\ & & & \end{array}$

